\title{
The geometry of $\mathrm{SO}(p) \times \mathrm{SO}(q)$-invariant special Lagrangian cones
}

\author{
Mark Haskins and Nikolaos Kapouleas
}

\begin{abstract}
$\mathrm{SO}(p) \times \mathrm{SO}(q)$-invariant special Lagrangian cones in $\mathbb{C}^{p+q}$ (equivalently, $\mathrm{SO}(p) \times \mathrm{SO}(q)$-invariant special Legendrians in $\left.\mathbb{S}^{2(p+q)-1}\right)$ are an important family of special Lagrangians (SL) whose basic features were studied in our previous paper [13]. In some ways, they play a role analogous to that of Delaunay surfaces in the geometry of CMC surfaces in $\mathbb{R}^{3}$; in particular, they are natural building blocks for our gluing constructions of higherdimensional SL cones $[9,10,12]$. In this article, we study in detail their geometry paying special attention to features needed in our gluing constructions. In particular, we classify them up to congruence; we determine their full group of symmetries (including various discrete symmetries) in all cases; we prove that many of them are closed and embedded; and finally understand the limiting singular geometry with detailed asymptotics. In understanding the detailed asymptotics a fundamental role is played by a certain conserved quantity (a component of the torque) considered in [13].
\end{abstract}

\section{Introduction}

Special Lagrangian (SL) $n$-folds in Calabi-Yau manifolds have been studied intensively over the past 15 years, thanks in part to their role in Mirror Symmetry [24]. Degenerations of families of smooth special Lagrangians and more general singular special Lagrangians play a crucial role, but in dimensions 3 and higher are still relatively poorly understood. SL cones in $\mathbb{C}^{n}$ with isolated singularities (or equivalently special Legendrians in $\mathbb{S}^{2 n-1}$ ) form the simplest class of singular special Lagrangians, and significant progress on understanding SL cones has been made in the last 10 years. In particular, the situation in dimension three has been clarified considerably $[3,6,8,11,15,21]$. By comparison the situation in higher dimensions is more complicated and less systematically explored.

In the current paper, we present a detailed study of the geometry of $\mathrm{SO}(p) \times \mathrm{SO}(q)$-invariant special Legendrians in $\mathbb{C}^{p+q}$ building on our previous work in [13]. They are some of the most basic special Legendrians 
and play a role similar to that of Delaunay surfaces in the geometry of CMC surfaces in $\mathbb{R}^{3}$. Like Delaunay surfaces, $\mathrm{SO}(p) \times \mathrm{SO}(q)$-invariant special Legendrians are cohomogeneity one objects and are therefore governed by an appropriate system of ODEs. For $(p, q)=(1,2)$ the $\mathrm{SO}(p) \times \mathrm{SO}(q)$ invariant special Legendrians are precisely the $\mathrm{SO}(2)$-invariant ones studied previously in $[6,8,11]$ and used as building blocks in our gluing construction of higher genus special Legendrian surfaces [11]. To our knowledge, for general $(p, q), \mathrm{SO}(p) \times \mathrm{SO}(q)$-invariant special Legendrians were first studied by Castro-Li-Urbano [4]. We pay particular attention to the following geometric features:

(i) the description of all $\mathrm{SO}(p) \times \mathrm{SO}(q)$-invariant special Legendrian immersions and their classification up to congruence;

(ii) the full isometry group of the induced metric for any $\mathrm{SO}(p) \times \mathrm{SO}(q)$ invariant special Legendrian immersion and its action on the domain;

(iii) the full group of symmetries (including various discrete symmetries) of any $\mathrm{SO}(p) \times \mathrm{SO}(q)$-invariant special Legendrian immersion;

(iv) the existence of sufficiently many closed embedded $\mathrm{SO}(p) \times \mathrm{SO}(q)$ invariant special Legendrians;

(v) the geometry and detailed asymptotics of the family of $\mathrm{SO}(p) \times \mathrm{SO}(q)$ invariant special Legendrians close to the equatorial sphere limit where the family degenerates.

These features play an essential role in the use of $\mathrm{SO}(p) \times \mathrm{SO}(q)$-invariant special Legendrians as building blocks in our higher-dimensional gluing constructions $[9,10,12]$.

Many features of the $\mathrm{SO}(2)$-invariant special Legendrians in $\mathbb{S}^{5}$ have analogues for $\mathrm{SO}(p) \times \mathrm{SO}(q)$-invariant special Legendrians in $\mathbb{S}^{2(p+q)-1}$. For instance, for each $(p, q)$ there is a real 1-parameter family of distinct $\operatorname{SO}(p) \times$ $\mathrm{SO}(q)$-invariant special Legendrian cylinders $X_{\tau}: \mathbb{R} \times \mathbb{S}^{p-1} \times \mathbb{S}^{q-1} \rightarrow$ $\mathbb{S}^{2(p+q)-1}$ depending real analytically on $\tau$ (see Proposition 3.30). The parameter $\tau$ can be identified with the value of some appropriately defined conserved quantity (a component of the torque). Geometrically, the parameter $\tau$ controls the size of the smallest orbit and hence the maximum of the absolute value of the curvature that occurs on the cylinder $X_{\tau}$; this value tends to infinity as $\tau \rightarrow 0$ and hence the family $X_{\tau}$ must degenerate in some way as $\tau \rightarrow 0$. In all our cases as $\tau \rightarrow 0, X_{\tau}$ approaches a necklace of equatorial $n-1$ spheres. In this sense, our building blocks are reminiscent of building blocks used in other gluing constructions-Delaunay surfaces in 
the construction of CMC surfaces in $\mathbb{R}^{3}[16-19]$ and Delaunay/Fowler metrics in the construction of constant scalar curvature metrics [22]. The fact that the family $X_{\tau}$ degenerates to a union of very simple geometric objects is fundamental to our gluing constructions in $[9,11,12]$.

For a given value of $p+q=n$, but different values of $p$ and $q$, the $\mathrm{SO}(p) \times \mathrm{SO}(q)$-invariant special Legendrian submanifolds all approach a necklace of equatorial $n-1$ spheres as $\tau \rightarrow 0$. For different values of $(p, q)$ these give rise to different kinds of spherical necklaces, in which the geometry of the transition regions that connects two adjacent almost spherical regions, and thus the relative positioning of adjacent almost spherical regions, changes. For $\mathrm{SO}(n-1)$-invariant special Legendrians each limiting equatorial sphere has two identical transition regions, which localize on two antipodal points of the equatorial sphere. Suitably enlarged the core of each of these transition regions resembles a Lagrangian catenoid as $\tau \rightarrow 0$. If $p>1$, the geometry of the transition regions of the $\mathrm{SO}(p) \times \mathrm{SO}(q)-$ invariant special Legendrians is more complicated. In this case, there are two different kinds of transition regions: one which localizes on a $(p-1)$ dimensional equatorial subsphere and another which localizes on a $(q-1)$ dimensional equatorial subsphere. In the former case, the core of the transition region resembles the product of a unit $p-1$ sphere with a small $q$-dimensional Lagrangian catenoid, and in the latter case the product of a small $p$-dimensional Lagrangian catenoid with a unit $q-1$ sphere. In the special case $p=q$, these two kinds of transition regions are isometric and there exist discrete symmetries that exchange the two kinds of transition regions; these symmetries cannot exist in the case $p \neq q$. The geometry of the almost spherical regions and the different kinds of transition regions are described in detail in Section 6.

The immersions $X_{\tau}$ give us special Legendrian immersions of the generalized cylinder $\mathbb{R} \times \mathbb{S}^{p-1} \times \mathbb{S}^{q-1}$. Let $g_{\tau}:=X_{\tau}^{*} g_{\mathbb{S}^{2 n-1}}$ denote the metric induced on the cylinder $\mathbb{R} \times \mathbb{S}^{p-1} \times \mathbb{S}^{q-1}$ by $X_{\tau}$. When $\tau=0 \quad X_{0}$ gives a diffeomorphism from the generalized cylinder $\mathbb{R} \times \mathbb{S}^{p-1} \times \mathbb{S}^{q-1}$ onto the standard real special Legendrian sphere $\mathbb{S}^{p+q-1} \subset \mathbb{S}^{2(p+q)-1}$ minus the union of the two equatorial subspheres $\left(\mathbb{S}^{p-1}, 0\right) \cup\left(0, \mathbb{S}^{q-1}\right) \subset \mathbb{R}^{p} \times \mathbb{R}^{q}$; in this case $g_{0}$ is isometric to the restriction of the standard metric on $\mathbb{S}^{p+q-1}$. When $|\tau|=\tau_{\max }$, the induced metric $g_{\tau}$ has a continuous translational symmetry and the immersion $X_{\tau}$ is not only $\mathrm{SO}(p) \times \mathrm{SO}(q)$-invariant but actually $\mathbb{S}^{1} \times \mathrm{SO}(p) \times \mathrm{SO}(q)$-invariant. In particular, $X_{ \pm \tau_{\max }}$ is homogeneous whereas the generic $X_{\tau}$ is only cohomogeneity one.

For $0<|\tau|<\tau_{\max }$ the metric $g_{\tau}$ induced on the cylinder is periodic of period $2 \mathrm{p}_{\tau}$. Translation by the period $2 \mathrm{p}_{\tau}$ induces a rotation of $X_{\tau}$ by 
some element $\widetilde{\mathrm{T}}_{2 \widehat{\mathrm{p}}_{\tau}} \in \mathrm{SU}(p+q)$ which we call the rotational period of $X_{\tau}$. In particular (as in the $\mathrm{SO}(2)$-invariant case), the angular period $\widehat{\mathrm{p}}_{\tau}$ (defined precisely in (4.23)) determines when $X_{\tau}$ factors through a closed special Legendrian embedding and hence gives rise to a SL cone in $\mathbb{C}^{n}$ with closed link. As $\tau \rightarrow 0$ the period $2 \mathrm{p}_{\tau}$ of the induced metric $g_{\tau}$ goes to infinity. In the $\tau \rightarrow 0$ limit, we see only a single "bead" (equatorial sphere) of the spherical necklace; the other beads of the necklace get pushed "beyond the horizon".

By studying the dependence of $\widehat{\mathbf{p}}_{\tau}$ on $\tau$ (see Proposition 7.41) and using the fact that we understand the limiting geometries when $\tau=0$ and $\tau=$ $\tau_{\max }$, we prove that for a dense set of $\tau, X_{\tau}$ factors as above. The behaviour of the angular period $\widehat{\mathrm{p}}_{\tau}$ and its derivative $\frac{d \hat{\mathrm{p}}_{\tau}}{d \tau}$ as $\tau \rightarrow 0$ is needed to understand quantitively how the geometry of $X_{\tau}$ changes when we make a small change in $\tau$ (and $\tau$ itself is also small). Understanding this behaviour is crucial to the gluing applications in [9-12].

The angular period $\widehat{p}_{\tau}$ can be expressed as an integral, which for $\mathrm{SO}(2)$ invariant SL cones is an elliptic integral [11, Equation (3.34) \& Appendix A]. In [11] we exploited results about elliptic integrals to prove our small $\tau$ asymptotics for $\frac{d \widehat{p}_{\tau}}{d \tau}[11,3.30]$. In higher dimensions $\widehat{p}_{\tau}$ has an expression in terms of hyperelliptic rather than elliptic integrals. In this paper, rather than studying the hyperelliptic integrals directly we adopt a more geometric approach that we expect will find application in other similar problems: every minimal submanifold of $\mathbb{S}^{m-1}$ has an associated homological invariant called the torque that arises directly from the First Variation Formula applied to Killing fields $\mathfrak{o}(m)$ of $\mathbb{S}^{m-1}$. We show that the torque detects the difference between the $X_{\tau}$ for different values of $\tau$.

Using the Legendrian neighbourhood theorem any Legendrian submanifold $C^{1}$-close to a given initial Legendrian submanifold $L$ can be described in terms of the 1-jet of some function $f$ on $L$; we denote this perturbed Legendrian submanifold $L_{f}$. The condition that a Legendrian submanifold $L$ be special Legendrian can be expressed in terms of the Legendrian angle $\theta_{\mathrm{L}}$, i.e., $\theta_{\mathrm{L}} \equiv 0$. Given an initial Legendrian immersion $L$ with Legendrian angle $\theta_{0}$, if the function $f$ is small then the Legendrian angle of the perturbed Legendrian $L_{f}$ has Legendrian angle $\theta_{0}+\mathcal{L} f+$ h.o.t. where

$$
\mathcal{L} f=(\Delta+2 n) f .
$$

We refer to $\mathcal{L} f$ as the linearized operator. By studying the linearization of (a suitable component of) the torque for small rotationally invariant perturbations of $X_{\tau}$ we derive an exact formula for the derivative $\frac{d \widehat{p}_{\tau}}{d \tau}$ in terms of the values of a distinguished rotationally invariant solution $Q$ to the linearized 
The geometry of $\mathrm{SO}(p) \times \mathrm{SO}(q)$-invariant special Lagrangian cones 175

equation $\mathcal{L} f=0$. This formula is valid for all values of $\tau$ not just small $\tau$ and may itself be useful for other purposes. By studying the behaviour of the distinguished solution $Q$ for small $\tau$, we are able to prove our result on the small $\tau$ asymptotics of $\frac{d \widehat{\mathrm{p}}_{\tau}}{d \tau}$ and $\widehat{\mathrm{p}}_{\tau}$.

Since the present paper builds on our previous work [13] some overlap with that paper is unavoidable. To make the current paper as self-contained as possible while minimizing overlap, we will state a number of results from [13] without proof; we will however introduce all necessary notation and terminology in the current paper, so that the reader need only consult [13] to see the proofs of these quoted results as necessary.

Organization of the paper. The paper is organized in seven sections. Section 1 consists of the introduction, this section and some remarks on notation. Section 2 contains some preliminary material of a more general nature related to symmetry groups of special Legendrian immersions. In Section 3, we recall some of the basic results about $\mathrm{SO}(p) \times \mathrm{SO}(q)$-invariant special Legendrians in $\mathbb{S}^{2 p+2 q-1}$ proved previously in [13]. These include the basic ODEs satisfied by (appropriately parameterized) $\mathrm{SO}(p) \times \mathrm{SO}(q)$ invariant special Legendrians (see (3.8)) and the 1-parameter family $X_{\tau}$ of $\mathrm{SO}(p) \times \mathrm{SO}(q)$-invariant special Legendrian immersions (see (3.29)); every $\mathrm{SO}(p) \times \mathrm{SO}(q)$-invariant special Legendrian is congruent to some $X_{\tau} . X_{\tau}$ is defined in terms of a 1-parameter family of solutions $\mathbf{w}_{\tau}$ to (3.8) which are described in (3.23). Section 4 studies the discrete symmetries of this 1-parameter family $\mathbf{w}_{\tau}$ of solutions of (3.8). We also introduce the periods and half-periods of $\mathbf{w}_{\tau}$; the periods of $\mathbf{w}_{\tau}$ control when $\mathbf{w}_{\tau}$ forms a closed curve in $\mathbb{S}^{3}$, while the half-periods control when the curve of isotropic $\mathrm{SO}(p) \times \mathrm{SO}(q)$ orbits associated with $\mathbf{w}_{\tau}$ is closed. The half-periods of $\mathbf{w}_{\tau}$ also control the embedding properties of $X_{\tau}$ (see Proposition 4.44). The discrete symmetries of $\mathbf{w}_{\tau}$ give rise to discrete symmetries of $X_{\tau}$ beyond the $\mathrm{SO}(p) \times \mathrm{SO}(q)$ symmetry implicit in the construction of $X_{\tau}$. Section 5 gives a complete analysis of all symmetries enjoyed by the 1-parameter family of $\mathrm{SO}(p) \times \mathrm{SO}(q)$-invariant special Legendrian immersions $X_{\tau}$. A symmetry of $X_{\tau}: \mathrm{Cyl}^{p, q} \rightarrow \mathbb{S}^{2 n-1}$ is a pair $(\mathrm{M}, \widetilde{\mathrm{M}}) \in \operatorname{Diff}\left(\mathrm{Cy}^{p, q}\right) \times \mathrm{O}(2 n)$ such that

$$
\widetilde{\mathrm{M}} \circ X_{\tau}=X_{\tau} \circ \mathrm{M} .
$$

If $(\mathrm{M}, \widetilde{\mathrm{M}})$ is any symmetry of $X_{\tau}$ then $\mathrm{M} \in \operatorname{Isom}\left(\mathrm{Cyl}^{p, q}, g_{\tau}\right)$, where $g_{\tau}:=$ $X_{\tau}^{*} g_{\mathbb{S}^{2 n-1}}$ is the pullback metric on $\mathrm{Cyl}^{p, q}$ induced by the immersion $X_{\tau}$ (see Remark 2.11). Proposition 5.6 determines the structure of the group Isom $\left(\mathrm{Cyl}^{p, q}, g_{\tau}\right)$. Subsequently, we show that in fact every element of $\operatorname{Isom}\left(\mathrm{Cyl}^{p, q}, g_{\tau}\right)$ gives rise to a symmetry of $X_{\tau}$. 
Section 6 studies the geometry of $X_{\tau}$ in detail. The first part of the section introduces subsets of $\mathrm{Cyl}^{p, q}$ called the waists and bulges of $X_{\tau}$ and associates to each bulge an equatorial $n-1$ sphere in $\mathbb{S}^{2 n-1}$ called its approximating sphere. We study the action of $\operatorname{Sym}\left(X_{\tau}\right)$ on the waists and bulges of $X_{\tau}$ and the action of $\widetilde{\operatorname{Sym}}\left(X_{\tau}\right)$ on the approximating spheres of $X_{\tau}$ (see Lemmas 6.8 and 6.13). The second part of the section studies the geometry of $X_{\tau}$ as $\tau \rightarrow 0$. We define subsets of the bulges, called almost spherical regions, and show that as $\tau \rightarrow 0$ the image of an almost spherical region under $X_{\tau}$ is close to its associated approximating sphere, thereby justifying the terminology. We also study the geometry of the necks of $X_{\tau}$-the core of the transition regions connecting two adjacent almost spherical regions centred around one of the waists - and show that as $\tau \rightarrow 0$ the necks approach a limiting geometry: if $p=1$ the necks all resemble small $(n-1)$-dimensional Lagrangian catenoids, while if $p>1$ there are two kinds of necks both of which resemble the product of a unit sphere with a small Lagrangian catenoid of the appropriate dimensions.

Section 7 studies the asymptotics of the angular period $\widehat{p}_{\tau}$ as $\tau \rightarrow 0$ refining the results we already proved in [13, Section 7]. To prove the small $\tau$ asymptotics of $\frac{d \widehat{\mathbf{p}}_{\tau}}{d \tau}$ we need Lemma 7.9, which calculates the linearization of the torque of $X_{\tau}$ when perturbed by a small rotationally invariant function $\phi$. Lemma 7.9 is a key ingredient of Lemma 7.24, which gives a precise formula for $\frac{d \widehat{\mathfrak{p}}_{\tau}}{d \tau}$ valid for any $0<\tau<\tau_{\max }$, in terms of the values of a particular solution to the rotationally invariant linearized operator (7.11). The small $\tau$ asymptotics of $\widehat{p}_{\tau}$ and $\frac{d \widehat{\mathbf{p}}_{\tau}}{d \tau}$ are easy consequences of this formula (see 7.41). As a corollary of the refined asymptotics of $\widehat{p}_{\tau}$ as $\tau \rightarrow 0$, we prove the existence of a countable dense set of $\tau$ for which $X_{\tau}$ factors through an embedding of a closed special Legendrian manifold (see 7.44).

Notation and conventions. Throughout the paper we use the following notation to express elements of $\operatorname{Isom}(\mathbb{R})$, the isometries of the real line. We denote by $\mathrm{T}_{x}$, translation by $x, t \mapsto t+x$. We denote by $\mathrm{I}$ reflection in the origin $t \mapsto-t$ and reflection in $x, t \mapsto 2 x-t$ by $\underline{\mathbf{T}}_{x}$.

\section{Preliminaries}

We assume the reader is familiar with the basic facts about SL geometry in $\mathbb{C}^{n}, \mathrm{SL}$ cones in $\mathbb{C}^{n}$ and special Legendrian submanifolds in $\mathbb{S}^{2 n-1}$. For the reader unfamiliar with these facts we refer them to [11, Section 2] for a brief introduction. 
The geometry of $\mathrm{SO}(p) \times \mathrm{SO}(q)$-invariant special Lagrangian cones 177

The following elementary group theory is used in several parts of the paper to describe the group structure of symmetry groups of our special Legendrian immersions; it appears below in our description of the Lagrangian and SL isometries of $\mathbb{C}^{n}$.

Direct, central and semidirect products of groups. Let $K, N \subset G$ be any two subsets of a group $G$. We write

$$
K N=\{k n \mid k \in K, n \in N\} \subset G .
$$

Sometimes we will also use the notation $K \cdot N$. If both $K$ and $N$ are subgroups of $G$, then $K N$ is a subgroup if and only if $K N=N K$ [14, p. 22]. Moreover, $N$ is a normal subgroup of $K N$ if and only if $k N k^{-1}=N$ for all $k \in K$ and similarly for $K$.

In particular, if $N$ centralizes $K$, i.e., every element of $N$ commutes with every element of $K$, then clearly $K N=N K$ and both $K$ and $N$ are normal subgroups of the group $H=K N$. In this case we have $K \cap N \subseteq Z(H)$, where $Z(H)$ denotes the centre of $H$, and we say that $K N$ is an (internal) central product of $K$ and $N$ identifying $K \cap N$ [5, p. 29]. If in fact $K \cap N=1$, then $K N$ is the (internal) direct product of $K$ and $N$, and $K N$ is isomorphic to the (external) direct product $K \times N$.

If $K$ is a normal subgroup of $K N$ then conjugation by any element of $N$ gives a homomorphism $\rho: N \rightarrow$ Aut $K$ and the kernel of $\rho$ is the centralizer of $K$ in $K N$. If $K$ is a normal subgroup of $K N$ and $K \cap N=1$, then $K N$ is the semidirect product of $K$ by $N, K \rtimes N$. To make explicit the conjugation action of $N$ on $K$ we often write, $K \rtimes_{\rho} N$ and write down the twisting homomorphism $\rho: N \rightarrow$ Aut $K$ explicitly.

Special Lagrangian isometries of $\mathbb{C}^{n}$. We recall some basic results on the structure of the subgroup of $\mathrm{O}(2 n)$ that maps the set of all SL $n$ planes in $\mathbb{C}^{n}$ to itself (possibly reversing the orientation of the plane). Let Lag denote the Grassmannian of unoriented Lagrangian $n$-planes in $\mathbb{C}^{n}$, SL denote the Grassmannian of (necessarily oriented) SL $n$-planes in $\mathbb{C}^{n}$ and define $\pm \mathrm{SL}:=\{\Pi \mid \pm \Pi \in \mathrm{SL}\}$.

\section{Definition 2.1 (Lagrangian and SL isometries).}

(i) Define $\mathrm{Isom}_{\text {Lag }}:=\{A \in \mathrm{O}(2 n) \mid A(\Pi) \in$ Lag for all $\Pi \in$ Lag $\}$. Elements of Isom $_{\text {Lag }}$ we call Lagrangian isometries.

(ii) Define $\operatorname{Isom}_{\mathrm{SL}}:=\{A \in \mathrm{O}(2 n) \mid A(\Pi) \in \mathrm{SL}$ for all $\Pi \in \mathrm{SL}\}$. Elements of IsomsL we call $S L$ isometries. 
(iii) Define $\mathrm{Isom}_{ \pm \mathrm{SL}}:=\{A \in \mathrm{O}(2 n) \mid A(\Pi) \in \pm$ SL for all $\Pi \in \mathrm{SL}\}$. Elements of $\mathrm{Isom}_{ \pm \mathrm{SL}}$ we call $\pm-S L$ isometries and elements of $\mathrm{Isom}_{ \pm \mathrm{SL}} \backslash \mathrm{Isom}_{\mathrm{SL}}$ we call anti-SL isometries.

Note we do not assume a priori that Isom $_{\text {SL }} \subset$ Isom $_{\text {Lag }}$, but from our definitions we do have Isom $_{\mathrm{SL}} \subset \mathrm{Isom}_{ \pm \mathrm{SL}}$.

Define $\mathrm{C} \in \mathrm{O}(2 n)$ by

$$
\mathrm{C}(z)=\bar{z}, \quad \text { where } z \in \mathbb{C}^{n} \text {. }
$$

Since C satisfies

$$
C^{*} J=-J, \quad C^{*} \omega=-\omega, \quad C^{*} \Omega=\bar{\Omega}
$$

we see in particular that $C$ belongs to both Isom $_{\text {Lag }}$ and Isom SL $_{\text {. }}$

We need the following result on the structure of Isom $_{\text {Lag }}$ and Isom $_{S L}$ to discuss the full symmetry group of $\mathrm{SO}(p) \times \mathrm{SO}(p)$-invariant special Legendrians.

\section{Lemma 2.3 (Structure of Isom $_{\mathrm{Lag}}$, Isom $\mathrm{SL}$ and Isom $_{ \pm \mathrm{SL}}$ ).}

(i) $\operatorname{Isom}_{L a g}=U(n) \cdot\langle\mathrm{C}\rangle \cong U(n) \rtimes_{\rho} \mathbb{Z}_{2}$ where the twisting homomorphism $\rho: \mathbb{Z}_{2} \rightarrow$ Aut $U(n)$ is determined by $\rho(1) U=\bar{U}$ for any $U \in U(n)$.

(ii) For $n>2$, we have

$$
\begin{aligned}
\operatorname{Isom}_{S L} & =S U(n) \cdot\langle\mathrm{C}\rangle \cong S U(n) \rtimes_{\rho} \mathbb{Z}_{2}, \\
\operatorname{Isom}_{ \pm S L} & =S U(n)^{ \pm} \cdot\langle\mathrm{C}\rangle \cong S U(n)^{ \pm} \rtimes_{\rho} \mathbb{Z}_{2},
\end{aligned}
$$

where

$$
S U(n)^{ \pm}:=\left\{U \in U(n) \mid \operatorname{det}_{\mathbb{C}} U= \pm 1\right\} \cong S U(n) \rtimes \mathbb{Z}_{2}
$$

and $\rho$ is the restriction of the twisting homomorphism defined in (i) to $S U(n)^{ \pm}$. In particular, $\mathrm{Isom}_{S L}$ and $\mathrm{Isom}_{ \pm S L}$ are subgroups of $\operatorname{Isom}_{\text {Lag }}$.

(iii) $\operatorname{Isom}_{S L}(2)=U_{I}(2)$ and $\operatorname{Isom}_{ \pm S L}=U_{I}(2) \cdot\left\langle\mathrm{R}_{1}\right\rangle \cong U_{I}(2) \rtimes_{\rho} \mathbb{Z}_{2}$ where $U_{I}(2)$ denotes the unitary group of $\mathbb{C}^{2}$ with respect to the complex structure $I$ on $\mathbb{C}^{2}$ defined by right multiplication by the imaginary quaternion $I \in \operatorname{Im} \mathbb{H}, \mathrm{R}_{1}\left(z_{1}, z_{2}\right):=\left(-z_{1}, z_{2}\right)$ and $\rho: \mathbb{Z}_{2} \rightarrow U_{I}(2)$ is the 
The geometry of $\mathrm{SO}(p) \times \mathrm{SO}(q)$-invariant special Lagrangian cones 179 homomorphism defined by $\rho(1) U=\mathrm{R}_{1} U \mathrm{R}_{1}$. $U_{I}(2)$ satisfies

$$
U_{I}(2) \cap U(2)=S U(2),
$$

where $U(2)$ and $S U(2)$ are the unitary and special unitary groups with respect to the standard complex structure $J$ on $\mathbb{C}^{2}$ (defined by right multiplication by $J \in \operatorname{Im} \mathbb{H}$ ). In particular, there exist $S L$ isometries of $\mathbb{C}^{2}$ which are not Lagrangian isometries.

We omit the proof. In fact, the following stronger version of Lemma 2.3 holds: if $n>2$ any diffeomorphism of $\mathbb{C}^{n}$ which preserves the SL differential ideal $\mathcal{I}$ generated by $\omega$ and $\operatorname{Im} \Omega$ is a product of a dilation with some element of $\operatorname{Isom}_{ \pm S L}[2]$.

In relation to (iii) we remark that using the standard basis $e_{1}=1, e_{2}=$ $I, e_{3}=J$ and $e_{4}=K$ for the quaternions $\mathbb{H} \cong \mathbb{C}^{2}$, the standard complex structure $J$ on $\mathbb{C}^{2}$ can be represented by the action of right multiplication by the unit imaginary quaternion $J \in \operatorname{Im}(\mathbb{H})$. With respect to the complex structure $I$ defined by right multiplication by the unit imaginary quaternion $I$ we have $\omega_{I}:=g(\cdot, I \cdot)=\operatorname{Re} \Omega_{J}$. Hence, the SL 2-planes of $\left(\mathbb{C}^{2}, J, \omega_{J}, \Omega_{J}\right)$ are exactly the $I$-complex lines in $\mathbb{C}^{2}$.

Corollary 2.4. If $n>2$ then any $S L$ isometry $\mathrm{L} \in \operatorname{Isom}_{S L}$ satisfies

$$
\mathrm{L}^{*} \omega=\omega, \quad \mathrm{L}^{*} \Omega=\Omega, \quad \text { or } \quad \mathrm{L}^{*} \omega=-\omega, \quad \mathrm{L}^{*} \Omega=\bar{\Omega},
$$

while any anti-SL isometry $\mathrm{L} \in \mathrm{Isom}_{ \pm S L} \backslash \operatorname{Isom}_{S L}$ satisfies

$$
\mathrm{L}^{*} \omega=\omega, \quad \mathrm{L}^{*} \Omega=-\Omega, \quad \text { or } \quad \mathrm{L}^{*} \omega=-\omega, \quad \mathrm{L}^{*} \Omega=-\bar{\Omega} .
$$

Corollary 2.4 implies that every \pm -SL isometry of $\mathbb{C}^{n}$ sends the complex structure $J$ to $\pm J$. In other words, every \pm -SL isometry of $\mathbb{C}^{n}$ is either a holomorphic or anti-holomorphic isometry of $\mathbb{C}^{n}$. Hence, we may also define another subgroup of $\mathrm{Isom}_{ \pm \mathrm{SL}}$ by

$$
\operatorname{Isom}_{ \pm \mathrm{SL}}^{J}:=\left\{A \in \mathrm{Isom}_{ \pm \mathrm{SL}} \mid A J=J A\right\}
$$

where $J$ denotes the standard complex structure on $\mathbb{C}^{n} .2 .3$ implies that

$$
\operatorname{Isom}_{ \pm \mathrm{SL}}^{J}=\operatorname{Isom}_{ \pm \mathrm{SL}} \cap \mathrm{U}(n)=\mathrm{SU}(n)^{ \pm} .
$$

Corollary 2.4 also implies that every SL isometry of $\mathbb{C}^{n}$ preserves the calibration $\operatorname{Re} \Omega$ and that every anti-SL isometry of $\mathbb{C}^{n}$ sends $\operatorname{Re} \Omega$ to $-\operatorname{Re} \Omega$. 
Symmetry groups and equivariance of special Legendrian immersions. For any special Legendrian immersion $X: M \rightarrow \mathbb{S}^{2 n-1}$ we define a symmetry of $X$ to be a pair $(\widetilde{\mathrm{M}}, \mathrm{M}) \in \mathrm{O}(2 n) \times \operatorname{Diff}(M)$ such that

$$
\tilde{\mathrm{M}} \circ X=X \circ \mathrm{M} \text {. }
$$

Note at this stage we only assume $\widetilde{\mathrm{M}} \in \mathrm{O}(2 n)$; however, in the cases of interest to us in the present paper we will see that $\widetilde{\mathrm{M}} \in \mathrm{Isom}_{ \pm \mathrm{SL}} \subset \mathrm{O}(2 n)$. The set of all symmetries of a special Legendrian immersion $X$ forms a group with the obvious multiplication. Rather than thinking of the symmetries of $X: M \rightarrow \mathbb{S}^{2 n-1}$ as a subgroup of $\mathrm{O}(2 n) \times \operatorname{Diff}(M)$ we often prefer to work with subgroups of the domain or target separately; this is helpful for example in finding all the symmetries of a given special Legendrian immersion $X$.

To this end we define a subgroup of $\operatorname{Diff}(M)$

$$
\operatorname{Sym}(X):=\{\mathrm{M} \in \operatorname{Diff}(M) \mid \exists \widetilde{\mathrm{M}} \in \mathrm{O}(2 n) \text { s.t. } \widetilde{\mathrm{M}} \circ X=X \circ \mathrm{M}\},
$$

and the subgroup $\operatorname{Per}(X) \subset \operatorname{Sym}(X)$ by

$$
\operatorname{Per}(X):=\{\mathrm{M} \in \operatorname{Diff}(M) \mid X \circ \mathrm{M}=X\} .
$$

It follows that if $\mathrm{M} \in \operatorname{Per}(X)$ then $\mathrm{M}$ must be orientation-preserving.

We also define a subgroup $\widetilde{\operatorname{Sym}}(X) \subset \operatorname{Isom}\left(\mathbb{S}^{2 n-1}\right)=\mathrm{O}(2 n)$ by

$$
\widetilde{\operatorname{Sym}}(X):=\{\widetilde{M} \in \mathrm{O}(2 n) \mid \widetilde{\mathrm{M}} \circ X=X \circ \mathrm{M} \text { for some } \mathrm{M} \in \operatorname{Sym}(X)\} .
$$

The three groups $\operatorname{Sym}(X), \operatorname{Per}(X)$ and $\widetilde{\operatorname{Sym}}(X)$ are related by the following.

Lemma 2.10. If $X: M \rightarrow \mathbb{S}^{2 n-1}$ is a Legendrian immersion that is not totally geodesic then there exists a canonical surjective homomorphism $\widetilde{\rho}$ : $\operatorname{Sym}(X) \rightarrow \widetilde{\operatorname{Sym}}(X)$ induced by sending $\mathrm{M} \in \operatorname{Sym}(X)$ to the (unique) $\widetilde{\mathrm{M}} \in$ $\widehat{\operatorname{Sym}}(X)$ such that $\widetilde{\mathrm{M}} \circ X=X \circ \mathrm{M}$. $\operatorname{ker} \widetilde{\rho}=\operatorname{Per}(X)$ and hence $\operatorname{Per}(X)$ is a normal subgroup of $\operatorname{Sym}(X)$ and

$$
\widetilde{\operatorname{Sym}}(X) \cong \operatorname{Sym}(X) / \operatorname{Per}(X) .
$$

Proof. Using the fact that any Legendrian submanifold of $\mathbb{S}^{2 n-1}$ that is not totally geodesic is linearly full [7, Lemma 3.13], one can see that if $\widetilde{\mathrm{M}}_{1}, \widetilde{\mathrm{M}}_{2} \in \mathrm{O}(2 n)$ and $\widetilde{\mathrm{M}}_{1} \circ X_{\tau}=\widetilde{\mathrm{M}}_{2} \circ X_{\tau}$ for some $\tau \neq 0$, then $\widetilde{\mathrm{M}}_{1}=\widetilde{\mathrm{M}}_{2}$. Hence, by the definitions of $\operatorname{Sym}\left(X_{\tau}\right)$ and $\widetilde{\operatorname{Sym}}\left(X_{\tau}\right)$, given any $\mathrm{M} \in \operatorname{Sym}\left(X_{\tau}\right)$ there exists a unique element $\widetilde{\mathrm{M}} \in \mathrm{O}(2 n)$ such that $\widetilde{\mathrm{M}} \circ X_{\tau}=X_{\tau} \circ \mathrm{M}$. We 
The geometry of $\mathrm{SO}(p) \times \mathrm{SO}(q)$-invariant special Lagrangian cones 181

define the map $\widetilde{\rho}: \operatorname{Sym}\left(X_{\tau}\right) \rightarrow \widetilde{\operatorname{Sym}}\left(X_{\tau}\right)$ by $\mathrm{M} \mapsto \widetilde{\mathrm{M}}$. $\widetilde{\rho}$ is readily seen to be a homomorphism, which by the definitions of $\operatorname{Sym}\left(X_{\tau}\right)$ and $\widetilde{\operatorname{Sym}}\left(X_{\tau}\right)$ is surjective. It follows immediately from the definition of $\operatorname{Per}\left(X_{\tau}\right)$ that $\operatorname{ker} \widetilde{\rho}=\operatorname{Per}\left(X_{\tau}\right)$.

Remark 2.11. For any $M \in \operatorname{Sym}(X)$ we observe that

$$
\begin{aligned}
\mathrm{M}^{*}\left(X^{*} g_{\mathbb{S}^{2 n-1}}\right) & =(X \circ \mathrm{M})^{*} g_{\mathbb{S}^{2 n-1}}=(\widetilde{\mathrm{M}} \circ X)^{*} g_{\mathbb{S}^{2 n-1}}=X^{*} \circ \widetilde{\mathrm{M}}^{*} g_{\mathbb{S}^{2 n-1}} \\
& =X^{*} g_{\mathbb{S}^{2 n-1}}
\end{aligned}
$$

Therefore any $\mathrm{M} \in \operatorname{Sym}(X)$ is an isometry of the pullback metric $g_{X}:=$ $X^{*} g_{\mathbb{S}^{2 n-1}}$ on $M$ and hence $\operatorname{Sym}(X)$ is always a subgroup of $\operatorname{Isom}\left(M, g_{X}\right)$; determining $\operatorname{Isom}\left(M, g_{X}\right)$ is thus a key step in determining $\operatorname{Sym}(X)$. For the cases of interest in this paper, we will see that in fact we always have $\operatorname{Sym}(X) \cong \operatorname{Isom}\left(M, g_{X}\right)$.

We have a monomorphism $\rho: \operatorname{Sym}(X) \rightarrow \operatorname{Diff}(M)$ given by inclusion and a homomorphism (not necessarily injective) $\widetilde{\rho}: \operatorname{Sym}(X) \rightarrow \mathrm{O}(2 n)$ given by $\mathrm{M} \mapsto \widetilde{\mathrm{M}}$ as in 2.10. Then by the definition of a symmetry of $X$ the immersion $X$ is $(\rho, \widetilde{\rho})$-equivariant, i.e.,

$$
\widetilde{\rho}(\gamma) \circ X=X \circ \rho(\gamma) \text { for all } \gamma \in \operatorname{Sym}(X) .
$$

We can talk about a $G$-equivariant immersion $X: M \rightarrow \mathbb{S}^{2 n-1}$ whenever we have a pair of homomorphisms $\rho: G \rightarrow \operatorname{Diff}(M)$ and $\widetilde{\rho}: G \rightarrow \mathrm{O}(2 n)$ by requiring that (2.12) hold for all $\gamma \in G$. For special Legendrian immersions it is natural to assume further that the homomorphism $\widetilde{\rho}$ satisfies $\widetilde{\rho}: G \rightarrow$ Isom $_{ \pm S \mathrm{SL}} \subset \mathrm{O}(2 n)$; this will always turn out to be the case for the symmetry groups considered in the current paper. In this case by 2.4 every element $\widetilde{\gamma}$ acts either holomorphically or anti-holomorphically on $\mathbb{C}^{n}$.

\section{Remark 2.13.}

(i) For any $\widetilde{\mathrm{M}} \in \mathrm{Isom}_{ \pm \mathrm{SL}}$ and any special Legendrian immersion $X: M \rightarrow$ $\mathbb{S}^{2 n-1}$ we can reposition $X$ using $\widetilde{M}$, i.e., (with an appropriate choice of orientation) $\widetilde{M} \circ X: M \rightarrow \mathbb{S}^{2 n-1}$ is another special Legendrian immersion. If additionally $X$ is $G$-equivariant and $\widetilde{\mathrm{M}}$ commutes with $G$ then $\widetilde{\mathrm{M}} \circ X$ is still $G$-equivariant. For this reason the centralizer of $G$, $C(G) \subset \mathrm{Isom}_{ \pm \mathrm{SL}}$ plays an important role in controlling the geometry of $G$-equivariant special Legendrians. 
(ii) If we assume $G \subset \mathrm{SU}(n)^{ \pm} \subset$ Isom $_{\mathrm{SL}}^{ \pm}$then clearly the centre $Z_{\mathrm{SU}(n)^{ \pm}}=$ $\left\langle\mathrm{e}^{\mathrm{i} \pi / n} \mathrm{Id}\right\rangle \cong \mathbb{Z}_{2 n}$ of $\mathrm{SU}(n)^{ \pm}$commutes with $G$ and hence can be used to give $G$-equivariant repositionings of any $G$-equivariant special Legendrian.

(iii) If we further assume $G \subset \mathrm{O}(n) \subset \mathrm{SU}(n)^{ \pm}$then $\mathrm{C}$ (defined in (2.2)) also commutes with $G$ (recall that conjugating by $\mathrm{C}$ acts on $\mathrm{GL}(n, \mathbb{C})$ by $\mathrm{M} \mapsto \overline{\mathrm{M}})$. Moreover conjugation by $\mathrm{C}$ acts on $Z_{\mathrm{SU}(n)^{ \pm}} \cong \mathbb{Z}_{2 n}$ by $\mathrm{e}^{\pi k \mathrm{i} / n} \mathrm{Id} \mapsto \mathrm{e}^{-\pi k \mathrm{i} / n} \mathrm{Id}$ and hence the group generated by $Z_{\mathrm{SU}(n)^{ \pm}} \cong \mathbb{Z}_{2 n}$ and the involution $\mathbf{C}$ is isomorphic to the dihedral group $\mathbf{D}_{2 n}$.

\section{3. $\mathrm{SO}(p) \times \mathrm{SO}(q)$-invariant special Legendrian submanifolds}

Given a pair of integers $p$ and $q$ satisfying $1 \leq p \leq q$ and $q \geq 2$ (we call such a pair $(p, q)$ admissible) we set $n=p+q$ and define cylinders of type $(p, q)$, $\mathrm{Cyl}_{I}^{p, q}$, by

$$
\mathrm{Cyl}_{I}^{p, q}:= \begin{cases}I \times \mathbb{S}^{p-1} \times \mathbb{S}^{q-1}, & \text { if } p>1 \\ I \times \mathbb{S}^{n-2}, & \text { if } p=1\end{cases}
$$

where $I \subset \mathbb{R}$ is an interval, which we omit in the notation when $I=\mathbb{R}$ and meridians $\operatorname{Mer}^{p, q}$ by

$$
\operatorname{Mer}^{p, q}:= \begin{cases}\mathbb{S}^{p-1} \times \mathbb{S}^{q-1}, & \text { if } p>1 \\ \mathbb{S}^{n-2}, & \text { if } p=1\end{cases}
$$

so that $\mathrm{Cyl}^{p, q}=\mathbb{R} \times \mathrm{Mer}^{p, q}$, i.e., $\mathrm{Mer}^{p, q}$ is the cross section of $\mathrm{Cyl}^{p, q}$.

$\mathrm{SO}(p) \times \mathrm{SO}(q)$ acts via isometries on $\mathbb{C}^{p+q} \cong \mathbb{C}^{p} \times \mathbb{C}^{q}$ via the product of the standard complex linear actions of $\mathrm{SO}(p)$ and $\mathrm{SO}(q)$ on the $\mathbb{C}^{p}$ and $\mathbb{C}^{q}$ factors respectively. Since $\mathrm{SO}(p) \times \mathrm{SO}(q) \subset \mathrm{SO}(p+q) \subset \mathrm{SU}(p+q)$ we can look for $\mathrm{SO}(p) \times \mathrm{SO}(q)$-invariant special Lagrangians in $\mathbb{C}^{p+q}$ and in particular for SL cones or equivalently special Legendrian submanifolds of $\mathbb{S}^{2(p+q)-1}$ invariant under $\mathrm{SO}(p) \times \mathrm{SO}(q)$.

If a Legendrian submanifold of $\mathbb{S}^{2 n-1}$ is a union of orbits then each orbit $\mathcal{O}$ must be $\gamma$-isotropic, i.e., $\left.\gamma\right|_{\mathcal{O}}=0$ where $\gamma$ denotes the standard contact form on $\mathbb{S}^{2 n-1}$. 
The geometry of $\mathrm{SO}(p) \times \mathrm{SO}(q)$-invariant special Lagrangian cones 183

\section{Lemma 3.3 (Isotropic orbits of $\mathrm{SO}(p) \times \mathrm{SO}(q)$ ).}

(i) If $p \geq 2, q \geq 2$ then any $\gamma$-isotropic $S O(p) \times S O(q)$ orbit $\mathcal{O} \subset \mathbb{S}^{2(p+q)-1}$ has the form

$$
\mathcal{O}_{\mathbf{w}}=\left(w_{1} \cdot \mathbb{S}^{p-1}, w_{2} \cdot \mathbb{S}^{q-1}\right)
$$

for some $\mathbf{w}=\left(w_{1}, w_{2}\right) \in \mathbb{S}^{3}$. Moreover, if $\mathbf{w}$ and $\mathbf{w}^{\prime} \in \mathbb{S}^{3}$ then $\mathcal{O}_{\mathbf{w}}=$ $\mathcal{O}_{\mathbf{w}^{\prime}}$ if and only if $\mathbf{w}^{\prime}=\rho_{j k} \mathbf{w}$ for some $(j, k) \in \mathbb{Z}_{2} \times \mathbb{Z}_{2}$ where $\rho: \mathbb{Z}_{2} \times$ $\mathbb{Z}_{2} \rightarrow O(2) \subset U(2)$ is the homomorphism defined by

$$
(j, k) \mapsto \rho_{j k}:=\left(\begin{array}{cc}
(-1)^{j} & 0 \\
0 & (-1)^{k}
\end{array}\right) .
$$

In particular, spherical isotropic $S O(p) \times S O(q)$ orbits are in one-toone correspondence with points in $\mathbb{S}^{3} / \mathbb{Z}_{2} \times \mathbb{Z}_{2}$.

(ii) Similarly, for $n \geq 3$ any $\gamma$-isotropic $S O(n-1)$ orbit $\mathcal{O} \subset \mathbb{S}^{2 n-1}$ has the form

$$
\mathcal{O}_{\mathbf{w}}=\left(w_{1}, w_{2} \cdot \mathbb{S}^{n-2}\right)
$$

for some $\mathbf{w}=\left(w_{1}, w_{2}\right) \in \mathbb{S}^{3}$. Moreover, if $\mathbf{w}$ and $\mathbf{w}^{\prime} \in \mathbb{S}^{3}$ then $\mathcal{O}_{\mathbf{w}}=$ $\mathcal{O}_{\mathbf{w}^{\prime}}$ if and only if $\mathbf{w}^{\prime}=\rho_{j k} \mathbf{w}$ for $(j, k) \in\langle(+-)\rangle \cong \mathbb{Z}_{2} \leqslant \mathbb{Z}_{2} \times \mathbb{Z}_{2}$. In particular, isotropic $S O(n-1)$ orbits in $\mathbb{S}^{2 n-1}$ are in one-to-one correspondence with points in $\mathbb{S}^{3} / \mathbb{Z}_{2}$, where $\mathbb{Z}_{2}=\left\langle\rho_{+-}\right\rangle$.

Proof. See [13, Lemma 3.1].

By Lemma 3.3 the generic $\gamma$-isotropic orbit of $\mathrm{SO}(p) \times \mathrm{SO}(q)$ has dimension $n-2$ and therefore we can look for $\mathrm{SO}(p) \times \mathrm{SO}(q)$-invariant special Legendrians that are curves of $\mathrm{SO}(p) \times \mathrm{SO}(q)$ orbits; these curves will satisfy a first order system of ODEs on the space of (generic) isotropic orbits and by 3.3 the space of all isotropic orbits can be identified with $\mathbb{S}^{3} / \mathbb{Z}_{2}$ if $p=1$ or $\mathbb{S}^{3} / \mathbb{Z}_{2} \times \mathbb{Z}_{2}$ if $p>1$. Locally one has existence and uniqueness for these ODEs but problems may develop if we run into singular orbits. To study the global geometry of the $\mathrm{SO}(p) \times \mathrm{SO}(q)$-invariant special Legendrians one needs to understand the possible singular orbits and how solutions to the ODEs behave on approach to these orbits. 


\section{Remark 3.6.}

(i) Recall from 2.13 the importance of the centralizer $C(G) \subset \operatorname{Isom}_{\mathrm{SL}}^{ \pm}$of the group $G \subset$ Isom $_{\mathrm{SL}}^{ \pm}$for the geometry of $G$-invariant special Legendrians. Of particular importance for the case $G=\mathrm{SO}(p) \times \mathrm{SO}(q) \subset$ $\mathrm{SO}(n)$ is the existence of the following 1-parameter subgroup $\left\{\widetilde{\mathrm{T}}_{x}\right\} \subset$ $\mathrm{SU}(n)$ commuting with $\mathrm{SO}(p) \times \mathrm{SO}(q)$ :

$$
\widetilde{\mathbf{T}}_{x}=\left(\begin{array}{cc}
\mathrm{e}^{\mathrm{i} x / p} \operatorname{Id}_{p} & 0 \\
0 & \mathrm{e}^{-\mathrm{i} x / q} \operatorname{Id}_{q}
\end{array}\right) .
$$

The 1-parameter subgroup $\left\{\widetilde{\mathbf{T}}_{x}\right\}$ plays a central role in governing the geometry of $\mathrm{SO}(p) \times \mathrm{SO}(q)$-invariant special Legendrians. In many ways it serves as an analogue of translations along the axis for the group $\mathrm{SO}(2) \subset$ Isom $\mathbb{E}^{3}$ of rotations around a fixed axis in $\mathbb{E}^{3}$. For this reason, we will often call repositioning an $\mathrm{SO}(p) \times \mathrm{SO}(q)$-invariant special Legendrian $X$ by some element $\widetilde{\mathrm{T}}_{x}$, "sliding" $X$ along its axis.

(ii) The function $f_{\mathbf{t}}: \mathbb{C}^{p+q} \rightarrow \mathbb{R}$ defined by

$$
f_{\mathbf{t}}(z, w)=\frac{1}{2 p}|z|^{2}-\frac{1}{2 q}|w|^{2},
$$

where $(z, w) \in \mathbb{C}^{p} \times \mathbb{C}^{q} \cong \mathbb{C}^{p+q}$, is the Hamiltonian function for the 1parameter subgroup $\left\{\widetilde{\mathrm{T}}_{x}\right\}$. Since $\left\{\widetilde{\mathrm{T}}_{x}\right\}$ commutes with $\mathrm{SO}(p) \times \mathrm{SO}(q)$ the function $f_{\mathbf{t}}$ generating $\left\{\widetilde{\mathrm{T}}_{x}\right\}$ is $\mathrm{SO}(p) \times \mathrm{SO}(q)$-invariant, i.e., satisfies $f \circ \mathrm{O}=f$ for any $\mathrm{O} \in \mathrm{SO}(p) \times \mathrm{SO}(q)$. Hence $f_{\mathbf{t}}$ is constant on any $\mathrm{SO}(p) \times \mathrm{SO}(q)$-orbit.

(iii) The standard special Legendrian equatorial sphere $\mathbb{S}^{n-1} \subset \mathbb{R}^{n} \subset \mathbb{C}^{n}$ is clearly $\mathrm{O}(n)$-invariant and also therefore $\mathrm{O}(p) \times \mathrm{O}(q)$-invariant. $\mathbb{S}^{n-1}$ is locally rigid as an $\mathrm{O}(n)$-invariant special Legendrian (although we can use the action of the centre $Z_{\mathrm{SU}(n)^{ \pm}}$to generate the other $\mathrm{O}(n)$ invariant special Legendrian equatorial spheres). However, as an $\mathrm{O}(p) \times$ $\mathrm{O}(q)$-invariant special Legendrian $\mathbb{S}^{n-1}$ is not rigid because we can reposition it using $\left\{\widetilde{\mathbf{T}}_{x}\right\}$, or "slide $\mathbb{S}^{n-1}$ along the axis of $\left\{\widetilde{\mathbf{T}}_{x}\right\}$ ". Namely, $\widetilde{\mathrm{T}}_{x}\left(\mathbb{S}^{n-1}\right)$ is a 1 -parameter family of $\mathrm{O}(p) \times \mathrm{O}(q)$-invariant special Legendrian equatorial $n-1$-spheres. The corresponding infinitesimal deformation of $\mathbb{S}^{n-1}$

$$
\left.\frac{d}{d x}\right|_{x=0} \widetilde{\mathbf{T}}_{x} \circ \mathbb{S}^{n-1}
$$


The geometry of $\mathrm{SO}(p) \times \mathrm{SO}(q)$-invariant special Lagrangian cones 185

gives rise to a solution to the linearized equation $\mathcal{L} f=(\Delta+2 n) f=0$, i.e., corresponds to a second harmonic $f$ on $\mathbb{S}^{n-1}$ which, since we are deforming through a 1-parameter family of $\mathrm{O}(p) \times \mathrm{O}(q)$-invariant special Legendrians must be $\mathrm{O}(p) \times \mathrm{O}(q)$-invariant. This $\mathrm{O}(p) \times \mathrm{O}(q)$ invariant second harmonic on $\mathbb{S}^{n-1}$ is $f(x, y)=\frac{1}{p} x^{2}-\frac{1}{q} y^{2}$ where $(x, y) \in \mathbb{S}^{p+q-1} \subset \mathbb{R}^{p} \times \mathbb{R}^{q}$, which is the restriction to $\mathbb{S}^{n-1}$ of the $\mathrm{O}(p) \times \mathrm{O}(q)$-invariant function $f_{\mathbf{t}}$ defined above.

With an appropriate parametrization the ODEs describing $\mathrm{SO}(p) \times$ $\mathrm{SO}(q)$-invariant special Legendrians are

$$
\begin{aligned}
& \dot{w}_{1}=\bar{w}_{1}^{p-1} \bar{w}_{2}^{q}, \\
& \dot{w}_{2}=-\bar{w}_{1}^{p} \bar{w}_{2}^{q-1} .
\end{aligned}
$$

In [13] we called solutions to $(3.8)(p, q)$-twisted $S L$ curves in $\mathbb{S}^{3}$. For any $p$ and $q$ the $(p, q)$-twisted SL ODEs (3.8) have six obvious types of symmetry:

(1) Time translation invariance $\mathbf{w} \mapsto \mathbf{w} \circ \mathrm{T}_{t_{0}}$ for any $t_{0} \in \mathbb{R}$.

(2) Multiplication by an $n$th root of unity $\mathbf{w} \mapsto z \mathbf{w}$, where $z^{n}=1$.

(3) $\mathbf{w} \mapsto \hat{\boldsymbol{T}}_{x} \circ \mathbf{w}$ where $\hat{\mathbf{T}}_{x} \in \mathrm{U}(1) \times \mathrm{U}(1) \subset \mathrm{U}(2)$ is the 1-parameter subgroup

$$
\hat{\mathrm{T}}_{x}=\left(\begin{array}{cc}
\mathrm{e}^{\mathrm{i} x / p} & 0 \\
0 & \mathrm{e}^{-\mathrm{i} x / q}
\end{array}\right) .
$$

(4) Complex conjugation $\mathbf{w} \mapsto \overline{\mathbf{w}}$.

(5) For any $n$th root of unity $z$ the simultaneous time reflection and spatial rotation given by

$$
t \mapsto-t, \quad \mathbf{w} \mapsto z \mathbf{w} .
$$

(6) The simultaneous time and spatial rescaling given by

$$
t \mapsto \lambda^{1-2 / n} t, \quad \mathbf{w} \mapsto \lambda^{1 / n} \mathbf{w}, \quad \text { for any } \lambda>0 .
$$

More precisely, $\mathbf{w}$ is a solution of (3.8) if and only if $\mathbf{w}_{\lambda}(t):=$ $\lambda^{1 / n} \mathbf{w}\left(\lambda^{1-2 / n} t\right)$ is. 
Finally, in the special case $p=q(3.8)$ is also invariant under

$$
t \mapsto-t, \quad\left(w_{1}, w_{2}\right) \mapsto\left(w_{2}, w_{1}\right)
$$

Symmetries (2-5) are manifestations of the symmetries of $\mathrm{SO}(p) \times \mathrm{SO}(q)$ invariant special Legendrians exhibited in Remarks 2.13 and 3.6.

The following result gives basic facts about solutions to the $(p, q)$-twisted SL ODEs.

\section{Proposition 3.10.}

(i) Solutions to the $(p, q)$-twisted SL ODEs (3.8) admit two conserved quantities

$$
\mathcal{I}_{1}(\mathbf{w}):=|\boldsymbol{w}|^{2} \quad \text { and } \quad \mathcal{I}_{2}(\mathbf{w}):=\operatorname{Im}\left(w_{1}^{p} w_{2}^{q}\right)
$$

The symmetries (1-3) preserve both conserved quantities $\mathcal{I}_{1}$ and $\mathcal{I}_{2}$. Symmetries (4) and (5) preserve $\mathcal{I}_{1}$ but send $\mathcal{I}_{2} \mapsto-\mathcal{I}_{2}$. Symmetry (6) sends $\left(\mathcal{I}_{1}, \mathcal{I}_{2}\right) \mapsto\left(\lambda^{2 / n} \mathcal{I}_{1}, \lambda \mathcal{I}_{2}\right)$. Hence if $\mathbf{w}$ is a solution of (3.8) with $\mathcal{I}_{1}(\mathbf{w}) \neq 0$ then we may rescale using symmetry $(6)$ to obtain another solution of (3.8) with $\mathcal{I}_{1}(\mathbf{w})=1$. For any solution with $\mathcal{I}_{1}(\boldsymbol{w})=1$, the possible range of values of $\mathcal{I}_{2}=\operatorname{Im}\left(w_{1}^{p} w_{2}^{q}\right)$ is $\left[-2 \tau_{\text {max }}, 2 \tau_{\text {max }}\right]$, where

$$
2 \tau_{\text {max }}=\sqrt{\frac{p^{p} q^{q}}{n^{n}}} .
$$

(ii) The stationary points of (3.8) are $\mathbb{C} \times\{0\} \cup\{0\} \times \mathbb{C}$ if $p>1$ or $\mathbb{C} \times$ $\{0\}$ if $p=1$.

(iii) The initial value problem for (3.8) with any initial data $\boldsymbol{w}(0) \in \mathbb{C}^{2}$ has a unique real analytic solution $\boldsymbol{w}: \mathbb{R} \rightarrow \mathbb{C}^{2}$ defined for all $t \in \mathbb{R}$, which depends real analytically on the initial data.

(iv) For any solution of (3.8) with $\mathcal{I}_{1}(\mathbf{w})=1$ and $\mathcal{I}_{2}(\mathbf{w})=\operatorname{Im}\left(w_{1}^{p} w_{2}^{q}\right)=$ $-2 \tau$ (and hence by part (i) $\tau \in\left[-\tau_{\max }, \tau_{\max }\right]$ ) the function $y:=\left|w_{2}\right|^{2}$ : $\mathbb{R} \rightarrow[0,1]$ satisfies the equation

$$
\frac{1}{2} \dot{y}+2 i \tau=-w_{1}^{p} w_{2}^{q}
$$

Therefore $y$ satisfies the energy conservation equation

$$
\dot{y}^{2}=4\left(f(y)-4 \tau^{2}\right)=4 y^{q}(1-y)^{p}-16 \tau^{2},
$$


The geometry of $\mathrm{SO}(p) \times \mathrm{SO}(q)$-invariant special Lagrangian cones 187

and hence also the second-order $O D E$

$$
\ddot{y}=2 f^{\prime}(y)=2 y^{q-1}(1-y)^{p-1}(q-n y),
$$

where we define the function $f: \mathbb{R} \rightarrow \mathbb{R}$ by

$$
f(y)=y^{q}(1-y)^{p} .
$$

(v) Any solution $\mathbf{w}$ of (3.8) satisfies

$$
|\dot{\mathbf{w}}|=\left|w_{1}\right|^{p-1}\left|w_{2}\right|^{q-1} .
$$

Proof. See $[13,4.7]$.

Remark 3.17. The difference between the stationary points of (3.8) in the case $p>1$ and the case $p=1$ reflects the difference in the geometry of the nongeneric isotropic orbits of $\mathrm{SO}(p) \times \mathrm{SO}(q)$ and $\mathrm{SO}(n-1)$ respectively. For $p>1$ the nongeneric isotropic orbits of $\mathrm{SO}(p) \times \mathrm{SO}(q)$ have the form $\left(w_{1} \cdot \mathbb{S}^{p-1}, 0\right)$ and $\left(0, w_{2} \cdot \mathbb{S}^{q-1}\right)$. For $p=1$ the only nongeneric isotropic orbits are of the form $\left(w_{1}, 0\right)$. In particular, the orbits of the form $\left(0, w_{2} \cdot \mathbb{S}^{n-2}\right)$ are generic provided $w_{2} \neq 0$.

We need the following auxiliary result about solutions of (3.13).

Lemma 3.18. Let $\mathbf{w}$ be any solution of (3.8) with $\mathcal{I}_{1}(\mathbf{w})=1$ and $\mathcal{I}_{2}(\mathbf{w})=$ $\operatorname{Im}\left(w_{1}^{p} w_{2}^{q}\right)=-2 \tau$ and let $y:=\left|w_{2}\right|^{2}: \mathbb{R} \rightarrow[0,1]$ be the associated solution of (3.13).

(i) If $0<|\tau|<\tau_{\max }$, the following holds:

(a) $y$ is periodic of period $2 \mathrm{p}_{\tau}>0$ and hence any two solutions of (3.13) with the same value of $\tau$ differ only by a time translation. Moreover, the period $\mathrm{p}_{\tau}$ satisfies

$$
\lim _{\tau \rightarrow \tau_{\max }} 2 \mathrm{p}_{\tau}=\frac{\pi}{\tau_{\max }} \sqrt{\frac{p q}{2 n^{3}}} .
$$

(b) The range of $y$ is $\left[y_{\min }, y_{\max }\right]$, where $0<y_{\min }<\frac{q}{n}<y_{\max }<1$ are the only two solutions of the degree $n$ polynomial equation

$$
f(y)=y^{q}(1-y)^{p}=4 \tau^{2},
$$

that lie in the interval $[0,1]$. 
(c) As $\tau \rightarrow 0$ we have

$$
y_{\min }=(2 \tau)^{2 / q}\left(1+O\left(\tau^{2 / q}\right)\right), \quad y_{\max }=1-(2 \tau)^{2 / p}\left(1+O\left(\tau^{2 / p}\right)\right) .
$$

(ii) If $|\tau|=\tau_{\max }$, then $y \equiv \frac{q}{n}$.

(iii) If $\tau=0$ and $p>1$ then one of the following holds:

(a) $y \equiv 0$,

(b) $y \equiv 1$,

(c) y is strictly monotone and satisfies,

$$
y= \begin{cases}y_{0} \circ \mathrm{T}_{t_{0}} & \text { some } t_{0} \in \mathbb{R} ; \quad \text { if } y \text { is decreasing }, \\ y_{0} \circ \mathrm{T}_{t_{0}} \circ \underline{\mathrm{I}} & \text { some } t_{0} \in \mathbb{R} ; \quad \text { if } y \text { is increasing }\end{cases}
$$

where $y_{0}: \mathbb{R} \rightarrow(0,1)$ denotes the unique (decreasing) solution to the initial value problem

$$
\dot{y}=-2 \sqrt{f(y)}, \quad y(0)=\frac{q}{n} .
$$

Alternatively, $y_{0}$ can be characterized as the unique solution to (3.14) with initial conditions

$$
y(0)=\frac{q}{n}, \quad \dot{y}(0)=-4 \tau_{\max }
$$

Moreover, $y_{0}$ satisfies $\lim _{t \rightarrow-\infty} y_{0}(t)=1$ and $\lim _{t \rightarrow \infty} y_{0}(t)=0$.

(iv) If $\tau=0$ and $p=1$ then one of the following holds:

(a) $y \equiv 0$,

(b) $y=y_{0} \circ \mathrm{T}_{t_{0}}$ for some $t_{0} \in \mathbb{R}$, where $y_{0}: \mathbb{R} \rightarrow(0,1]$ is the unique solution to (3.14) with initial conditions $y(0)=1, \quad \dot{y}(0)=0$. Moreover, $y_{0}$ is even, increasing on $(-\infty, 0)$ and satisfies $\lim _{t \rightarrow \pm \infty} y_{0}(t)=0$.

Proof. See [13, Lemma 4.19].

We have the following normal forms for solutions of (3.8).

Proposition 3.22. Fix a pair of admissible integers $p$ and $q$ and let $\mathbf{w}$ be any solution of (3.8) with $\mathcal{I}_{1}(\mathbf{w})=1$ and $\mathcal{I}_{2}(\mathbf{w})=-2 \tau$ with $0 \leq|\tau| \leq \tau_{\text {max }}$.

(i) If $p>1$ and $0<|\tau| \leq \tau_{\max }$ then $\mathbf{w}$ is equivalent under symmetries (1)-(3) to $\mathbf{w}_{\tau}: \mathbb{R} \rightarrow \mathbb{S}^{3}$ defined as the unique solution to (3.8) with 
The geometry of $\mathrm{SO}(p) \times \mathrm{SO}(q)$-invariant special Lagrangian cones 189 initial value

$$
\mathbf{w}_{\tau}(0)=\left(\sqrt{\frac{p}{n}} \mathrm{e}^{\mathrm{i} \alpha_{\tau} / 2 p}, \sqrt{\frac{q}{n}} \mathrm{e}^{\mathrm{i} \alpha_{\tau} / 2 q}\right)
$$

where $\alpha_{\tau} \in\left[-\frac{\pi}{2}, \frac{\pi}{2}\right]$ is defined by

$$
\alpha_{\tau}:=\arcsin \left(-\frac{\tau}{\tau_{\max }}\right)
$$

(ii) If $p>1$ and $\tau=0$ then $\mathbf{w}$ is equivalent under symmetries (1)-(3) to the unique solution of (3.8) with one of the following four initial conditions:
(a) $\mathbf{w}(0)=(1,0)$,
(b) $\mathbf{w}(0)=(0,1)$,
(c) $\mathbf{w}(0)=\left(\sqrt{\frac{p}{n}}, \sqrt{\frac{q}{n}}\right)$,
(d) $\mathbf{w}(0)=\left(\mathrm{e}^{\mathrm{i} \pi / 2 p} \sqrt{\frac{p}{n}}, \mathrm{e}^{\mathrm{i} \pi / 2 q} \sqrt{\frac{q}{n}}\right)$.

(iii) If $p=1$ and $0<|\tau| \leq \tau_{\max }$ then $\mathbf{w}$ is equivalent under symmetries (1)-(3) to $\mathbf{w}_{\tau}: \mathbb{R} \rightarrow \mathbb{S}^{3}$ defined as the unique solution to (3.8) with initial value

$$
\mathbf{w}_{\tau}(0)=\left(-i \operatorname{sgn} \tau \sqrt{1-y_{\max }}, \sqrt{y_{\max }}\right) .
$$

(iv) If $p=1$ and $\tau=0$ then $\mathbf{w}$ is equivalent under symmetries (1)-(3) to the unique solution of (3.8) with one of the following two initial conditions:
(a) $\mathbf{w}(0)=(1,0)$,
(b) $\mathbf{w}(0)=(0,1)$.

Proof. See [13, Prop 4.26]

$\mathrm{w}_{\tau}$ and the $\mathrm{SO}(p) \times \mathrm{SO}(q)$-invariant special Legendrian immersions $\boldsymbol{X}_{\boldsymbol{\tau}}$. We now define the particular 1-parameter family of $(p, q)$-twisted SL curves we will use throughout the rest of the paper by specifying initial data $\mathbf{w}_{\tau}(0)$ as in the normal form given in 3.22. Associated to the 1-parameter family $\mathbf{w}_{\tau}$ is the 1-parameter family $X_{\tau}$ of $\mathrm{SO}(p) \times \mathrm{SO}(q)$ invariant special Legendrians. Proposition 3.22 implies that any $\mathrm{SO}(p) \times$ $\mathrm{SO}(q)$-invariant special Legendrian in $\mathbb{S}^{2(p+q)-1}$ is congruent to $X_{\tau}$ for some $\tau$.

Proposition 3.23. Fix a pair of admissible integers $p$ and $q$ and choose any $\tau \in\left[-\tau_{\max }, \tau_{\max }\right]$. Define $\mathbf{w}_{\tau}: \mathbb{R} \rightarrow \mathbb{S}^{3}$ as the unique solution of (3.8) 
with initial data

$$
\mathbf{w}_{\tau}(0)=\left(\sqrt{\frac{p}{n}} \mathrm{e}^{\mathrm{i} \alpha_{\tau} / 2 p}, \sqrt{\frac{q}{n}} \mathrm{e}^{\mathrm{i} \alpha_{\tau} / 2 q}\right) \quad \text { if } p>1 ;
$$

where $\alpha_{\tau} \in\left[-\frac{\pi}{2}, \frac{\pi}{2}\right]$ is defined by

$$
\alpha_{\tau}:=\arcsin \left(-\frac{\tau}{\tau_{\max }}\right),
$$

or

$$
\mathbf{w}_{\tau}(0)=\left(-i \operatorname{sgn} \tau \sqrt{1-y_{\max }}, \sqrt{y_{\max }}\right) \quad \text { if } p=1
$$

Then $\mathbf{w}_{\tau}$ depends real analytically on $\tau \in\left(-\tau_{\max }, \tau_{\max }\right)$ and satisfies $\mathbf{w}_{-\tau}=$ $\overline{\mathbf{w}}_{\tau}$. In particular, $\mathbf{w}_{0}: \mathbb{R} \rightarrow \mathbb{S}^{3} \subset \mathbb{C}^{2}$ is contained in $\mathbb{R}^{2} \subset \mathbb{C}^{2}$.

Proof. See [13, Prop 5.1].

The associated function $y_{\tau}:=\left|w_{2}\right|^{2}$ and its initial value characterization. For the solution $\mathbf{w}_{\tau}$ defined in (3.23), define $y_{\tau}:=\left|w_{2}\right|^{2}$. By (3.10) $y_{\tau}$ satisfies equations (3.13) and (3.14). Analytic dependence of $y_{\tau}$ on $\tau \in$ $\left(-\tau_{\max }, \tau_{\max }\right)$ follows immediately from analytic dependence of $\mathbf{w}_{\tau}$.

For $p=1, y_{\tau}$ is the unique solution of (3.14) satisfying the initial conditions

$$
y(0)=y_{\max }, \quad \dot{y}(0)=0 \text {. }
$$

In particular, $y_{0}$ is the unique solution of (3.14) satisfying $y(0)=1, \dot{y}(0)=0$ introduced in 3.18.iv.b.

Similarly, for $p>1, y_{\tau}$ is the unique solution of (3.14) satisfying the initial conditions

$$
y(0)=\frac{q}{n}, \quad \dot{y}(0)=-4 \tau_{\max } \cos \alpha_{\tau}=-4 \sqrt{\tau_{\max }^{2}-\tau^{2}} .
$$

$y_{0}$ coincides with the solution of (3.14) satisfying $y(0)=q / n, \dot{y}(0)=-4 \tau_{\max }$ introduced in 3.18.iii.c.

For both $p=1$ and $p>1$ it follows from these initial value characterizations of $y_{\tau}$ that $y_{-\tau}=y_{\tau}$, which is consistent with the fact that $\mathbf{w}_{-\tau}=\overline{\mathbf{w}}_{\tau}$.

We now define the family of special Legendrian immersions $X_{\tau}: \mathrm{Cyl}^{p, q} \rightarrow$ $\mathbb{S}^{2(p+q)-1}$ using the $(p, q)$-twisted SL curves $\boldsymbol{w}_{\tau}$ defined in Proposition 3.23, where $\mathrm{Cyl}^{p, q}$ denotes the cylinder of type $(p, q)$ defined in (3.1). 
The geometry of $\mathrm{SO}(p) \times \mathrm{SO}(q)$-invariant special Lagrangian cones 191

Definition 3.29. For $\tau \in\left[-\tau_{\max }, \tau_{\max }\right]$ define an immersion $X_{\tau}$ : Cyl ${ }^{p, q} \rightarrow$ $\mathbb{S}^{2(p+q)-1}$ by

$$
\begin{aligned}
X_{\tau}\left(t, \sigma_{1}, \sigma_{2}\right) & =\left(w_{1}(t) \cdot \sigma_{1}, w_{2}(t) \cdot \sigma_{2}\right), \quad \text { for } p>1 ; \\
X_{\tau}(t, \sigma) & =\left(w_{1}(t), w_{2}(t) \cdot \sigma\right), \quad \text { for } p=1,
\end{aligned}
$$

where $t \in \mathbb{R}, \sigma_{1} \in \mathbb{S}^{p-1}, \sigma_{2} \in \mathbb{S}^{q-1}, \sigma \in \mathbb{S}^{n-2}$ and $\mathbf{w}_{\tau}=\left(w_{1}, w_{2}\right)$ is the unique solution to (3.8) specified in Proposition 3.23.

We now state the basic properties of $X_{\tau}$.

Proposition 3.30. For $\tau \in\left[-\tau_{\max }, \tau_{\max }\right]$ the immersion $X_{\tau}: \mathrm{Cyl}^{p, q} \rightarrow$ $\mathbb{S}^{2(p+q)-1}$ defined in (3.29) has the following properties:

(i) $X_{\tau}$ is a smooth special Legendrian immersion depending analytically on $\tau$ for $\tau \in\left(-\tau_{\max }, \tau_{\max }\right)$, and satisfies $X_{-\tau}=\bar{X}_{\tau}$. In particular, $X_{0}$ is contained in $\mathbb{S}^{p+q-1} \subset \mathbb{R}^{p+q} \subset \mathbb{C}^{p+q}$.

(ii) For $p>1$, the metric $g_{\tau}$ on $\mathrm{Cyl}^{p, q}$ induced by $X_{\tau}$ is

$$
\begin{aligned}
& |\dot{\mathbf{w}}|^{2} d t^{2}+\left|w_{1}\right|^{2} g_{\mathbb{S}^{p-1}}+\left|w_{2}\right|^{2} g_{\mathbb{S}^{q-1}} \\
& \quad=y^{q-1}(1-y)^{p-1} d t^{2}+(1-y) g_{\mathbb{S}^{p-1}}+y g_{\mathbb{S}^{q-1}} .
\end{aligned}
$$

For $p=1$, the induced metric $g_{\tau}$ on $\mathrm{Cyl}^{1, n-1}$ is

$$
|\dot{\mathbf{w}}|^{2} d t^{2}+\left|w_{2}\right|^{2} g_{\mathbb{S}^{n-2}}=y^{n-2} d t^{2}+y g_{\mathbb{S}^{n-2}} .
$$

(iii) $X_{\tau}$ is $S O(p) \times S O(q)$-equivariant, i.e., for any $\mathrm{O}=\left(\mathrm{O}_{1}, \mathrm{O}_{2}\right) \in S O(p) \times$ $S O(q)$ we have

$$
\widetilde{\mathrm{O}} \circ X_{\tau}=X_{\tau} \circ \mathrm{O},
$$

where $\mathrm{O}=\left(\mathrm{O}_{1}, \mathrm{O}_{2}\right)$ acts on $\mathrm{Cyl}^{p, q}$ by $\mathrm{O} \cdot\left(t, \sigma_{1}, \sigma_{2}\right)=\left(t, \mathrm{O}_{1} \sigma_{1}, \mathrm{O}_{2} \sigma_{2}\right)$, and

$$
\widetilde{\mathrm{O}}=\left(\begin{array}{cc}
\mathrm{O}_{1} & 0 \\
0 & \mathrm{O}_{2}
\end{array}\right) \in S O(p) \times S O(q) \subset S O(p+q) \subset S U(p+q) .
$$

(iv) When $\tau=0$ we have

$$
X_{0}\left(\mathrm{Cyl}^{p, q}\right)= \begin{cases}\mathbb{S}^{p+q-1} \backslash\left(\mathbb{S}^{p-1}, 0\right) \cup\left(0, \mathbb{S}^{q-1}\right), & \text { for } p>1 ; \\ \mathbb{S}^{n-1} \backslash( \pm 1,0) \in \mathbb{R} \oplus \mathbb{R}^{n-1}, & \text { for } p=1 .\end{cases}
$$


(v) When $\tau=\tau_{\max }$, we have

$$
X_{\tau_{\max }}(t, \sigma)=\left(-i \sqrt{\frac{1}{n}} e^{2 i n \tau t}, \sqrt{\frac{n-1}{n}} e^{-2 i n \tau t /(n-1)} \sigma\right), \quad \text { for } p=1
$$

$$
\begin{aligned}
X_{\tau_{\max }}\left(t, \sigma_{1}, \sigma_{2}\right)= & \left(\sqrt{\frac{p}{n}} \mathrm{e}^{-\mathrm{i} \pi /(4 p)} e^{2 n i \tau t / p} \sigma_{1}, \sqrt{\frac{q}{n}} \mathrm{e}^{-\mathrm{i} \pi /(4 q)} e^{-2 n i \tau t / q} \sigma_{2}\right), \\
& \text { for } p>1 .
\end{aligned}
$$

(vi) If $X: \mathrm{Cyl}^{p, q} \rightarrow \mathbb{S}^{2(p+q)-1}$ is any non totally geodesic $S O(p) \times S O(q)$ invariant special Legendrian immersion then $X=\mathrm{e}^{\mathrm{i} \omega} \widetilde{\mathrm{T}}_{x} \circ X_{\tau} \circ \mathrm{T}_{y}$ for some $x, y \in \mathbb{R}, 0<|\tau|<\tau_{\max }$ and nth root of unity $\omega \in \mathbb{S}^{1}$ with $\widetilde{\mathrm{T}}_{x} \in$ $S U(n)$ as defined in (3.7).

Proof. See [13, Prop 5.9].

Torques of $\boldsymbol{X}_{\boldsymbol{\tau}}$. The value of the conserved quantity $\mathcal{I}_{2}\left(\mathbf{w}_{\tau}\right)=-2 \tau$ on the solution $\mathbf{w}_{\tau}$ has a natural geometric interpretation in terms of the value of a torque associated with the special Legendrian $X_{\tau}$; torques are certain conserved quantities defined for any special Legendrian (in fact for any minimal submanifold of a round sphere) and associated with the rotational symmetries of the sphere. Torques will play an important role in Section 7, where we use them to understand in a quantitative way how the geometry of $X_{\tau}$ changes when $\tau$ is sufficiently close to 0 and we make a small (relative to the size of $\tau$ ) change in $\tau$ : see $7.9,7.24$ and 7.41 .

Suppose $M$ is an oriented $m$-dimensional submanifold of the ambient manifold $(\bar{M}, \bar{g})$ and $\mathrm{k} \in \mathfrak{i s o}(\bar{M}, \bar{g})$ is a Killing field on $(\bar{M}, \bar{g})$. Given any oriented hypersurface $\Sigma \subset M$ we define the k-flux through $\Sigma$ by

$$
\mathcal{F}_{\mathrm{k}}(\Sigma):=\int_{\Sigma} \bar{g}(\mathrm{k}, \eta) d \mathrm{v}_{\Sigma}
$$

where $\eta$ is the unit conormal to $\Sigma$, chosen so that the orientation defined by $\Sigma$ and $\eta$ agrees with that of $M$. An immediate consequence of the First Variation of Volume formula $[23,7.6]$ is

Lemma 3.33. If $M$ is an oriented $m$-dimensional minimal submanifold of $(\bar{M}, \bar{g}), \Sigma$ is an oriented hypersurface of $M$ and $\mathrm{k} \in \mathfrak{i s o}(\bar{M}, \bar{g})$ then the $\mathrm{k}$-flux through $\Sigma, \mathcal{F}_{\mathrm{k}}(\Sigma)$, depends only on the homology class $[\Sigma] \in H_{m-1}(M, \mathbb{R})$. 
The geometry of $\mathrm{SO}(p) \times \mathrm{SO}(q)$-invariant special Lagrangian cones 193

In other words, when $M$ is a minimal submanifold of $(\bar{M}, \bar{g})$ the k-flux map defined in (3.32) induces a linear map $\mathcal{F}: H_{m-1}(M, \mathbb{R}) \rightarrow \mathfrak{i s o}(\bar{M}, \bar{g})^{*}$.

If $(\bar{M}, \bar{g})=\left(\mathbb{S}^{2 n-1}, g_{\text {std }}\right)$ then iso $(\bar{M}, \bar{g})=\mathfrak{o}(2 n)$ and we call the map $\mathcal{F}: H_{m-1}(M, \mathbb{R}) \rightarrow \mathfrak{o}(2 n)^{*}$ the torque of $M$. For special Legendrian submanifolds of $\mathbb{S}^{2 n-1}$ it is also convenient to define the restricted torque of $M$, which is the restriction of the torque to the subalgebra $\mathfrak{s u}(n) \subset \mathfrak{o}(2 n)$.

Proposition 3.34. For $p>1$ the $\mathfrak{s u}(n)$ restricted torque of the $S O(p) \times$ $S O(q)$-invariant special Legendrian immersion $X_{\tau}: \mathrm{Cyl}^{p, q} \rightarrow \mathbb{S}^{2(p+q)-1}$ is given by

$$
\mathcal{F}_{\mathrm{k}}\left(X_{\tau}\right)=\left\{\begin{array}{c}
2 \tau\left(\frac{1}{p} \sum_{i=1}^{p} \lambda_{i}-\frac{1}{q} \sum_{j=1}^{q} \mu_{j}\right) \operatorname{Vol}\left(\mathbb{S}^{p-1}\right) \operatorname{Vol}\left(\mathbb{S}^{q-1}\right) \\
\text { if } \mathrm{k}=i \operatorname{diag}\left(\lambda_{1}, \ldots, \lambda_{p}, \mu_{1}, \ldots, \mu_{q}\right) \\
0 \quad \text { if } \mathrm{k} \in \mathfrak{s u}(n) \text { is off-diagonal, }
\end{array}\right.
$$

where we implicitly use the homology class of any meridian in $\mathrm{Cy}^{p, q}$.

For $p=1$ the $\mathfrak{s u}(n)$ restricted torque of the $S O(n-1)$-invariant special Legendrian immersion $X_{\tau}: \mathrm{Cyl}^{1, n-1} \rightarrow \mathbb{S}^{2 n-1}$ is given by

$$
\mathcal{F}_{\mathbf{k}}\left(X_{\tau}\right)= \begin{cases}2 \tau\left(\lambda-\frac{1}{n-1} \sum_{j=1}^{n-1} \mu_{j}\right) \operatorname{Vol}\left(\mathbb{S}^{n-2}\right) & \mathrm{k}=i \operatorname{diag}\left(\lambda, \mu_{1}, \ldots, \mu_{n-1}\right) ; \\ 0 & \mathrm{k} \in \mathfrak{s u}(n) \text { is off-diagonal. }\end{cases}
$$

In particular, if we take $\mathrm{k}=\mathrm{t}$ to be the generator of the 1-parameter subgroup $\left\{\widetilde{\mathbf{T}}_{x}\right\}$ (defined in (3.7)) then we obtain

$$
\mathcal{F}_{\mathrm{t}}\left(X_{\tau}\right)= \begin{cases}2 \tau \frac{n}{p q} \operatorname{Vol}\left(\mathbb{S}^{p-1}\right) \operatorname{Vol}\left(\mathbb{S}^{q-1}\right), & \text { if } p>1 ; \\ 2 \tau \frac{n}{n-1} \operatorname{Vol}\left(\mathbb{S}^{n-2}\right), & \text { if } p=1 ;\end{cases}
$$

and hence the value of the torque $\mathcal{F}_{\mathrm{t}}$ gives another characterization of the parameter $\tau$.

Proof. See [13, Prop 5.14].

\section{Discrete symmetries of $\mathrm{w}_{\tau}$}

In this section, we study the discrete symmetries of $\mathbf{w}_{\tau}$ and the conditions under which $\mathbf{w}_{\tau}$ corresponds to a closed curve of $\mathrm{SO}(p) \times \mathrm{SO}(q)$ orbits. We 


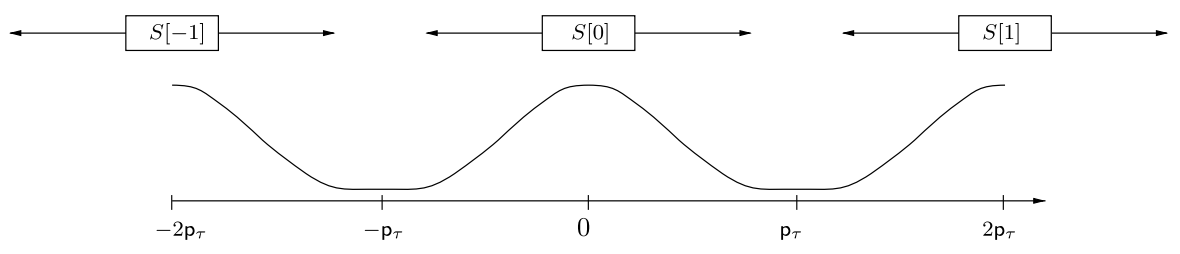

Figure 1: Profile of $y_{\tau}:=\left|w_{2}\right|^{2}$ for $p=1$.

will use these results in the following section to study the full group of symmetries of $X_{\tau}$.

Symmetries of $\boldsymbol{y}_{\tau}$. We begin by describing the symmetries of $y_{\tau}:=\left|w_{2}\right|^{2}$. To state these results we need to introduce some notation to describe the basic properties of $y_{\tau}$. For $p>1$, recall from (3.28) that $y_{\tau}$ satisfies the initial conditions

$$
y(0)=\frac{q}{n}, \quad \dot{y}(0)=-4 \tau_{\max } \cos \alpha_{\tau}=-4 \sqrt{\tau_{\max }^{2}-\tau^{2}},
$$

whereas for $p=1$ from (3.27) it satisfies

$$
y(0)=y_{\max }, \quad \dot{y}(0)=0 .
$$

The different initial conditions for $y_{\tau}$ affect where the $2 \mathrm{p}_{\tau}$-periodic function $y_{\tau}$ attains its maxima and minima in the cases $p=1$ and $p>1$. In the case $p>1$ the choice of initial data for $y_{\tau}$ implies that there exist unique real numbers $\mathrm{p}_{\tau}^{+}, \mathrm{p}_{\tau}^{-} \in\left(0, \mathbf{p}_{\tau}\right)$ satisfying

$$
y_{\tau}\left(-\mathbf{p}_{\tau}^{-}\right)=y_{\max }, \quad y_{\tau}\left(\mathbf{p}_{\tau}^{+}\right)=y_{\min },
$$

and so that $y_{\tau}$ is strictly decreasing on $\left(-\mathrm{p}_{\tau}^{-}, \mathrm{p}_{\tau}^{+}\right)$. We call these two numbers the partial-periods of $y_{\tau}$, since

$$
2 \mathrm{p}_{\tau}=2 \mathrm{p}_{\tau}^{+}+2 \mathrm{p}_{\tau}^{-} .
$$

In general, $\mathrm{p}_{\tau}^{+}$and $\mathrm{p}_{\tau}^{-}$are not related except when $p=q$ when we will prove shortly that they are equal. Illustrative plots of $y_{\tau}$ are shown in figures 1 and 2 for $p=1$ and $p>1, p \neq q$, respectively.

Throughout the following lemma we assume $0<|\tau|<\tau_{\max }$ and discuss the exceptional cases $\tau=0$ and $|\tau|=\tau_{\max }$ in Remark 4.12 below. Recall, also the notation for elements in $\operatorname{Isom}(\mathbb{R})$ introduced in Section 1 in Notation and Conventions. 
The geometry of $\mathrm{SO}(p) \times \mathrm{SO}(q)$-invariant special Lagrangian cones 195

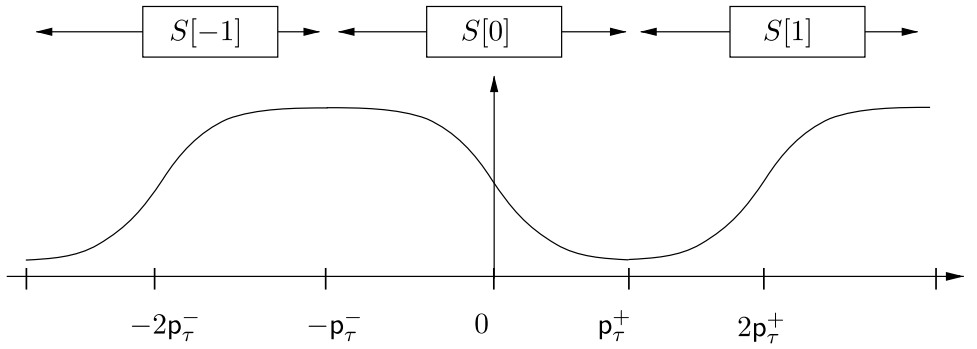

Figure 2: Profile of $y_{\tau}=\left|w_{2}\right|^{2}$ for $p>1$.

\section{Lemma 4.3 (Symmetries of $y_{\tau}$ ).}

(i) For $p=1, q=n-1$ the symmetries of $y_{\tau}=\left|w_{2}\right|^{2}$ are generated by

$$
y_{\tau} \circ \mathrm{T}_{2 \mathbf{p}_{\tau}}=y_{\tau} \quad \text { and } \quad y_{\tau} \circ \underline{\mathrm{T}}=y_{\tau} \text {. }
$$

That is, $y_{\tau}$ is an even $2 \mathrm{p}_{\tau}$-periodic function. Moreover, we have

$$
y_{\tau}(0)=y_{\max } \quad \text { and } \quad y_{\tau}\left(\mathrm{p}_{\tau}\right)=y_{\min }
$$

(ii) For $p>1$ and $p \neq q$ the symmetries of $y_{\tau}$ are generated by

$$
y_{\tau} \circ \mathrm{T}_{2 \mathbf{p}_{\tau}}=y_{\tau}, \quad y_{\tau} \circ \underline{\mathbf{T}}_{\mathbf{p}_{\tau}^{+}}=y_{\tau} \quad \text { and } \quad y_{\tau} \circ \underline{\mathrm{T}}_{-\mathbf{p}_{\tau}^{-}}=y_{\tau} .
$$

(iii) For $p>1$ and $p=q$ the symmetries of $y_{\tau}$ are generated by

$$
\begin{aligned}
y_{\tau} \circ \mathrm{T}_{2 \mathbf{p}_{\tau}} & =y_{\tau}, \quad y_{\tau} \circ \underline{\mathbf{T}}_{\mathbf{p}_{\tau} / 2}=y_{\tau}, \quad y_{\tau} \circ \underline{\mathbf{T}}_{-\mathbf{p}_{\tau} / 2}=y_{\tau} \quad \text { and } \\
y_{\tau} \circ \underline{\mathbf{T}} & =1-y_{\tau},
\end{aligned}
$$

and the partial-periods defined in (4.2) satisfy

$$
\mathbf{p}_{\tau}^{+}=\mathbf{p}_{\tau}^{-}=\frac{1}{2} \mathbf{p}_{\tau} \quad \text { and } \quad y_{\tau}\left(\frac{1}{2} \mathbf{p}_{\tau}\right)=y_{\min }, \quad y_{\tau}\left(-\frac{1}{2} \mathbf{p}_{\tau}\right)=y_{\max }
$$

Proof. See [13, Lemma 6.3].

Remark 4.9. It follows from the partial-period relation (4.2) that the reflections $\underline{\mathrm{T}}_{\mathbf{p}_{\tau}^{+}}$and $\underline{\mathrm{T}}_{-\mathrm{p}_{\tau}^{-}}$satisfy

$$
\underline{\mathrm{T}}_{-\mathrm{p}_{\tau}^{-}} \circ \underline{\mathrm{T}}_{\mathrm{p}_{\tau}^{+}}=\mathrm{T}_{-2 \mathrm{p}_{\tau}}, \quad \underline{\mathrm{T}}_{\mathrm{p}_{\tau}^{+}} \circ \underline{\mathrm{T}}_{-\mathrm{p}_{\tau}^{-}}=\mathrm{T}_{2 \mathrm{p}_{\tau}}
$$

Hence, the first symmetry of $y_{\tau}$ in (4.6) is a consequence of the second and third symmetries. 
Similarly, it is straightforward to check that $\underline{\mathrm{I}} \underline{\mathrm{T}}_{\mathrm{p}_{\tau} / 2} \circ \underline{\mathrm{T}}=\underline{\mathrm{I}}_{-\mathrm{p}_{\tau} / 2}$. It follows that the two symmetries $\underline{T}$ and $\underline{T}_{p_{\tau} / 2}$ are sufficient to generate all four symmetries in (4.7). The fourth symmetry in (4.7) implies that for $p=q$ we have

$$
y_{\min }+y_{\max }=1 \text {. }
$$

Remark 4.12. For $\tau=0, y_{\tau}$ is no longer periodic (the period $2 \mathrm{p}_{\tau} \rightarrow \infty$ as $\tau \rightarrow 0$ ). For $p=1$, we have already seen in 3.18.iv.b that $y_{0}$ is still even. For $p=q, y_{0}(0)$ is invariant under $y \mapsto 1-y$, and hence $y_{0}$ retains the reflectional symmetry

$$
y_{0} \circ \underline{\mathrm{T}}=1-y_{0}
$$

When $|\tau|=\tau_{\max }, y_{\tau}$ is the constant function $q / n$, as noted in Proposition 3.18.

Corollary 4.13. The discrete subgroup $\mathbf{D}$ of $\operatorname{Isom}(\mathbb{R})$ generated by the symmetries of $y_{\tau}$ is

$$
\mathbf{D}= \begin{cases}\left\langle\underline{\mathrm{T}}, \mathrm{T}_{2 \mathbf{p}_{\tau}}\right\rangle & \text { if } p=1 \\ \left\langle\underline{\mathbf{T}}_{\mathbf{p}_{\tau}^{+}}, \underline{\mathbf{T}}_{-\mathbf{p}_{\tau}^{-}}\right\rangle & \text {if } p>1, p \neq q ; \\ \left\langle\underline{\mathbf{T}}, \underline{\mathbf{T}}_{\mathbf{p}_{\tau} / 2}\right\rangle & \text { if } p>1, p=q .\end{cases}
$$

In all three cases $\mathbf{D} \cong \mathbf{D}_{\infty}$ the infinite dihedral group.

Proof. Recall the two standard presentations for the infinite dihedral group $\mathbf{D}_{\infty}$

$$
\left\langle r, f \mid f^{2}=1, f r f=r^{-1}\right\rangle, \quad \text { and } \quad\left\langle s, t \mid s^{2}=1, t^{2}=1\right\rangle .
$$

The commutation relation

$$
\underline{\mathrm{T}} \circ \mathrm{T}_{x} \circ \underline{\mathrm{T}}=\mathrm{T}_{-x}
$$

together with the first presentation of $\mathbf{D}_{\infty}$ shows that $\mathbf{D} \cong \mathbf{D}_{\infty}$ in the case $p=1$. The commutation relations $(4.10)$ for the reflection symmetries $\underline{\mathrm{T}}_{\mathbf{p}_{\tau}^{+}}$ and $\underline{I}_{-p_{\tau}^{-}}$together with the second presentation of $\mathbf{D}_{\infty}$ yield the result for $p>1$ and $p \neq q$. Similarly, for $p=q, \mathbf{D}$ is a group generated by two independent reflections $s$ and $t$ with no relation of the form $(s t)^{k}=1$, hence isomorphic to $\mathbf{D}_{\infty}$. 
The geometry of $\mathrm{SO}(p) \times \mathrm{SO}(q)$-invariant special Lagrangian cones 197

Symmetries of $\mathbf{w}_{\boldsymbol{\tau}}$. In this subsection we study the symmetries of $\mathbf{w}_{\tau}$. Since $X_{\tau}$ is determined by $\mathbf{w}_{\tau}$ these symmetries are intimately connected to the extrinsic geometry of $X_{\tau}$. The symmetries of $\mathbf{w}_{\tau}$ are themselves closely related to the symmetries of $y_{\tau}$ studied in the previous section. Since by Propositions 3.23 and 3.30.i, $\mathbf{w}_{-\tau}=\overline{\mathbf{w}}_{\tau}$ and $X_{-\tau}=\bar{X}_{\tau}$, it suffices to consider only $\tau \geq 0$.

It follows from (3.8) and the fact that $\mathcal{I}_{2}\left(\mathbf{w}_{\tau}\right)=\operatorname{Im} w_{1}^{p} w_{2}^{q}=-2 \tau$ that

$$
\operatorname{Im}\left(\bar{w}_{1} \dot{w}_{1}\right)=-\operatorname{Im}\left(\bar{w}_{2} \dot{w}_{2}\right)=2 \tau .
$$

If $\mathbf{w}_{\tau}=\left(w_{1}, w_{2}\right), y_{\tau}=\left|w_{2}\right|^{2}$ and $\psi_{1}$ and $\psi_{2}$ denote the arguments of $w_{1}$ and $w_{2}$ respectively then the previous equations are equivalent to

$$
\left(1-y_{\tau}\right) \dot{\psi}_{1}=2 \tau, \quad y_{\tau} \dot{\psi}_{2}=-2 \tau .
$$

To study the symmetries of $\mathbf{w}_{\tau}$ it is convenient to write $\mathbf{w}_{\tau}$ in the form

$$
\begin{aligned}
& w_{1}(t)= \begin{cases}\operatorname{sgn} t \sqrt{1-y_{0}(t)}, & \text { for } \tau=0 ; \\
-i \sqrt{1-y_{\tau}(t)} \mathrm{e}^{\mathrm{i} \psi_{1}}, & \text { for } \tau>0 ;\end{cases} \\
& w_{2}(t)= \begin{cases}\sqrt{y_{0}(t)}, & \text { for } \tau=0 ; \\
\sqrt{y_{\tau}(t)} \mathrm{e}^{\mathrm{i} \psi_{2}}, & \text { for } \tau>0 ;\end{cases}
\end{aligned}
$$

if $p=1$ and

$$
\begin{aligned}
& w_{1}(t)= \begin{cases}\sqrt{1-y_{0}(t)}, & \text { for } \tau=0 ; \\
\sqrt{1-y_{\tau}(t)} \mathrm{e}^{\mathrm{i} \alpha_{\tau} / 2 p} \mathrm{e}^{\mathrm{i} \psi_{1}}, & \text { for } \tau>0 ;\end{cases} \\
& w_{2}(t)= \begin{cases}\sqrt{y_{0}(t)}, & \text { for } \tau=0 ; \\
\sqrt{y_{\tau}(t)} \mathrm{e}^{\mathrm{i} \alpha_{\tau} / 2 q} \mathrm{e}^{\mathrm{i} \psi_{2}}, & \text { for } \tau>0 ;\end{cases}
\end{aligned}
$$

if $p>1$, where $\alpha_{\tau} \in[-\pi / 2, \pi / 2]$ was defined in (3.25) and where in both cases for $0<\tau \leq \tau_{\max }, \psi_{1}, \psi_{2}: \mathbb{R} \rightarrow \mathbb{R}$ are the unique solutions of (4.15) with initial conditions

$$
\psi_{1}(0)=\psi_{2}(0)=0 .
$$

The slightly different forms the above $w_{i}$ take in the cases $p=1$ and $p>$ 1 stem from the fact that we have chosen the initial data $\mathbf{w}(0)$ for $\mathbf{w}_{\tau}$ differently in these two cases (recall (3.24) and (3.26)). 
Define the function $\Psi$ by

$$
\Psi:=p \psi_{1}+q \psi_{2}
$$

Written in terms of $y$ and $\Psi$ the real and imaginary parts of equation (3.12) are equivalent to

$$
\begin{aligned}
& \dot{y}_{\tau}=-2 \sqrt{f(y)} \sin \Psi, \\
& 2 \tau=\sqrt{f(y)} \cos \Psi,
\end{aligned}
$$

for $p=1$ and to

$$
\begin{aligned}
\dot{y}_{\tau} & =-2 \sqrt{f(y)} \cos \left(\Psi+\alpha_{\tau}\right), \\
-2 \tau & =\sqrt{f(y)} \sin \left(\Psi+\alpha_{\tau}\right),
\end{aligned}
$$

for $p>1$ with $\alpha_{\tau}$ as defined in (3.25).

Definition 4.22. For any $\tau$ with $0<|\tau|<\tau_{\max }$ we define the angular period $\widehat{\mathrm{p}}_{\tau}$ in terms of $\psi_{1}$ by

$$
2 \widehat{\mathrm{p}}_{\tau}:=p \psi_{1}\left(2 \mathrm{p}_{\tau}\right)
$$

In $\left[13\right.$, Prop 7.7] we proved that the angular period $2 \widehat{\mathrm{p}}_{\tau}$ is a nonconstant analytic function of $\tau$ for $0<|\tau|<\tau_{\max }$ that satisfies

$$
\lim _{\tau \rightarrow 0} \widehat{\mathbf{p}}_{\tau}=\frac{\pi}{2} .
$$

More precise asymptotics for $\widehat{\mathrm{p}}_{\tau}$ as $\tau \rightarrow 0$ will be important in our subsequent gluing constructions and will be established in Section 7 .

Lemma 4.25 (Discrete symmetries of $\mathbf{w}_{\boldsymbol{\tau}}$ for $\boldsymbol{p}=\mathbf{1}$ ). For $p=1, q=$ $n-1$ and $0<\tau<\tau_{\max }$ the angular period $\widehat{\mathrm{p}}_{\tau}$ defined in (4.23) satisfies

(4.26) $2 \widehat{\mathrm{p}}_{\tau}:=\psi_{1}\left(2 \mathrm{p}_{\tau}\right)=2 \psi_{1}\left(\mathrm{p}_{\tau}\right)=-2(n-1) \psi_{2}\left(\mathrm{p}_{\tau}\right)=-(n-1) \psi_{2}\left(2 \mathrm{p}_{\tau}\right)$.

$\mathbf{w}_{\tau}$ has the following symmetries:

$$
\mathbf{w}_{\tau} \circ \mathrm{T}_{2 \mathbf{p}_{\tau}}=\hat{\mathrm{T}}_{2 \widehat{p}_{\tau}} \circ \mathbf{w}_{\tau}, \quad \mathbf{w}_{\tau} \circ \underline{\mathrm{I}}=\underline{\hat{\mathbf{T}}} \circ \mathbf{w}_{\tau}, \quad \mathbf{w}_{\tau} \circ \underline{\mathrm{T}}_{\mathrm{p}_{\tau}}=\hat{\mathrm{T}}_{2 \widehat{\mathrm{p}}_{\tau}} \circ \underline{\hat{\mathbf{T}}} \circ \mathbf{w}_{\tau},
$$

where $\hat{\mathrm{T}}_{x} \in U(2)$ was defined in (3.9) and $\hat{\mathbf{T}} \in O(4)$ is defined by

$$
\hat{\mathbf{I}}\left(w_{1}, w_{2}\right)=\left(-\bar{w}_{1}, \bar{w}_{2}\right) \text {. }
$$


The geometry of $\mathrm{SO}(p) \times \mathrm{SO}(q)$-invariant special Lagrangian cones 199

Using the fact that $\overline{\mathbf{w}}_{\tau}=-\mathbf{w}_{\tau}$ the symmetries of $\mathbf{w}_{\tau}$ for $\tau<0$ can be inferred immediately from the symmetries in the case $\tau>0$.

We have the following analogue of Lemma 4.25 for $p>1$.

Lemma 4.28 (Discrete symmetries of $\mathrm{w}_{\boldsymbol{\tau}}$ for $\boldsymbol{p}>1$ ). Fix a pair of admissible integers $p$ and $q$ with $p>1$, then for $0<\tau<\tau_{\max }$, the angular period $\widehat{\mathrm{p}}_{\tau}$ satisfies

$$
\begin{aligned}
2 \widehat{\mathbf{p}}_{\tau} & :=p \psi_{1}\left(2 \mathbf{p}_{\tau}\right)=2 p\left(\psi_{1}\left(\mathbf{p}_{\tau}^{+}\right)-\psi_{1}\left(-\mathbf{p}_{\tau}^{-}\right)\right) \\
& =-2 q\left(\psi_{2}\left(\mathbf{p}_{\tau}^{+}\right)-\psi_{2}\left(-\mathbf{p}_{\tau}^{-}\right)\right)=-q \psi_{2}\left(2 \mathbf{p}_{\tau}\right) .
\end{aligned}
$$

$\mathbf{w}_{\tau}$ has the following symmetries:

$$
\mathbf{w}_{\tau} \circ \mathrm{T}_{2 \mathbf{p}_{\tau}}=\hat{\mathrm{T}}_{2 \widehat{\mathbf{p}}_{\tau}} \circ \mathbf{w}_{\tau}, \quad \mathbf{w}_{\tau} \circ{\underline{\mathbf{I}_{\mathrm{p}}^{+}}}_{\mathrm{p}_{+}}=\hat{\underline{I}}_{+} \circ \mathbf{w}_{\tau}, \quad \mathbf{w}_{\tau} \circ \underline{I}_{-\mathbf{p}_{\tau}^{-}}=\underline{\hat{I}}_{-} \circ \mathbf{w}_{\tau},
$$

where $\hat{\mathrm{T}}_{x} \in U(2)$ was defined in (3.9) and $\hat{\mathbf{I}}_{+}, \hat{\mathbf{I}}_{-} \in O(4)$ are defined by

$$
\begin{aligned}
& \hat{\mathbf{I}}_{+}\left(w_{1}, w_{2}\right)=\left(\mathrm{e}^{\mathrm{i} \alpha_{\tau} / p} \mathrm{e}^{\mathrm{i} \psi_{1}\left(2 \mathbf{p}_{\tau}^{+}\right)} \bar{w}_{1}, \mathrm{e}^{\mathrm{i} \alpha_{\tau} / q} \mathrm{e}^{\mathrm{i} \psi_{2}\left(2 \mathbf{p}_{\tau}^{+}\right)} \bar{w}_{2}\right), \\
& \hat{\mathbf{I}}_{-}\left(w_{1}, w_{2}\right)=\left(\mathrm{e}^{\mathrm{i} \alpha_{\tau} / p} \mathrm{e}^{\mathrm{i} \psi_{1}\left(-2 \mathbf{p}_{\tau}^{-}\right)} \bar{w}_{1}, \mathrm{e}^{\mathrm{i} \alpha_{\tau} / q} \mathrm{e}^{\mathrm{i} \psi_{2}\left(-2 \mathbf{p}_{\tau}^{-}\right)} \bar{w}_{2}\right) .
\end{aligned}
$$

When $p=q, \mathbf{w}_{\tau}$ has the following extra symmetry:

$$
w_{1} \circ \underline{\mathbf{T}}=w_{2} \quad \text { and } \quad w_{2} \circ \underline{\mathbf{T}}=w_{1} .
$$

Hence $\psi_{1}$ and $\psi_{2}$ have the following additional symmetries:

$$
\begin{aligned}
\psi_{1} \circ \underline{\mathbf{I}} & =\psi_{2}, \quad \psi_{2} \circ \underline{\mathbf{I}}=\psi_{1}, \quad \psi_{1} \circ \mathbf{T}_{\mathbf{p}_{\tau}}=-\psi_{2}+\psi_{1}\left(\mathbf{p}_{\tau}\right), \\
\psi_{2} \circ \mathbf{T}_{\mathbf{p}_{\tau}} & =-\psi_{1}+\psi_{2}\left(\mathbf{p}_{\tau}\right) .
\end{aligned}
$$

The angular period $\widehat{\mathrm{p}}_{\tau}$ satisfies

$$
2 \widehat{\mathbf{p}}_{\tau}:=p \psi_{1}\left(2 \mathbf{p}_{\tau}\right)=p\left(\psi_{1}\left(\mathbf{p}_{\tau}\right)-\psi_{1}\left(-\mathbf{p}_{\tau}\right)\right)=-p \psi_{2}\left(2 \mathbf{p}_{\tau}\right) .
$$

The proofs of Lemmas 4.25 and 4.28 are very similar. First, we establish symmetries of $\psi_{i}$ using the symmetries of $y_{\tau}$ from Lemma 4.3 together with the definitions of $\psi_{i}$ in terms of $y_{\tau}$ (recall (4.15)). The symmetries of $\mathbf{w}_{\tau}$ then follow by using the definition of $\mathbf{w}_{\tau}$ in terms of $y_{\tau}$ and $\psi_{i}$ and their symmetries. For completeness, we give details in each case. 
Proof of Lemma 4.25. Proof of (4.26) and (4.27): The discrete symmetries of $y_{\tau}$ given in (4.4) and the definition of $\psi_{i}$ in terms of $y_{\tau}$ given in (4.15) imply the following symmetries for $\psi_{i}(i=1,2)$

$$
\psi_{i} \circ \mathrm{T}_{2 \mathrm{p}_{\tau}}=\psi_{i}+\psi_{i}\left(2 \mathrm{p}_{\tau}\right), \quad \psi_{i} \circ \underline{\mathrm{I}}=-\psi_{i}, \quad \psi_{i} \circ \underline{\mathrm{T}}_{\mathbf{p}_{\tau}}=-\psi_{i}+\psi_{i}\left(2 \mathrm{p}_{\tau}\right) .
$$

Proof of (4.26): $\psi_{i}\left(2 \mathrm{p}_{\tau}\right)=2 \psi_{i}\left(\mathrm{p}_{\tau}\right)$ for $i=1,2$ follows from the third symmetry of (4.34) when $t=\mathrm{p}_{\tau}$. It remains to prove that $\Psi\left(\mathrm{p}_{\tau}\right)=\psi_{1}\left(\mathrm{p}_{\tau}\right)+$ $(n-1) \psi_{2}\left(\mathbf{p}_{\tau}\right)=0$. Since $\Psi(0)=0$ and $\sqrt{f(y)(t)}$ is continuous in $t$ and positive, (4.20b) implies that $\Psi(t) \in\left(-\frac{\pi}{2}, \frac{\pi}{2}\right)$ for all $t \in \mathbb{R}$. Then since $\dot{y}\left(\mathrm{p}_{\tau}\right)=0$, from (3.13) it follows that $\sqrt{f(y)\left(\mathrm{p}_{\tau}\right)}=2 \tau$ and hence from (4.20b) that $\cos (\Psi)\left(\mathrm{p}_{\tau}\right)=1$ as required.

The symmetries of $\psi_{i}$ given in (4.34), together with the fact that $\Psi\left(2 \mathrm{p}_{\tau}\right)=2 \Psi\left(\mathrm{p}_{\tau}\right)=0$, imply the following simpler symmetries for $\Psi$

$$
\Psi \circ \mathrm{T}_{2 \mathrm{p}_{\tau}}=\Psi, \quad \Psi \circ \underline{\mathrm{T}}=-\Psi, \quad \Psi \circ \underline{\mathrm{T}}_{\mathrm{p}_{\tau}}=-\Psi
$$

In other words (unlike $\psi_{1}$ or $\psi_{2}$ individually), $\Psi$ is an odd periodic function of $t$ of period $2 \mathrm{p}_{\tau}$.

Proof of (4.27): The symmetries of $\mathbf{w}_{\tau}$ claimed in (4.27) follow from (4.4), (4.26) and (4.34) and the expression (4.16) for $\mathbf{w}_{\tau}$ in terms of $y_{\tau}, \psi_{1}$ and $\psi_{2}$.

Proof of Lemma 4.28. Symmetries of $\psi_{i}$ : the symmetries of $y_{\tau}$ given in (4.6) and the definition of $\psi_{i}$ in terms of $y_{\tau}$ given in (4.15) imply the following symmetries for $\psi_{i}(i=1,2)$ :

$$
\begin{aligned}
\psi_{i} \circ \underline{\mathrm{T}}_{\mathrm{p}_{\tau}^{+}} & =-\psi_{i}+\psi_{i}\left(2 \mathrm{p}_{\tau}^{+}\right), \quad \psi_{i} \circ \underline{\mathrm{T}}_{-\mathrm{p}_{\tau}^{-}}=-\psi_{i}+\psi_{i}\left(-2 \mathrm{p}_{\tau}^{-}\right), \\
\psi_{i} \circ \mathrm{T}_{2 \mathrm{p}_{\tau}} & =\psi_{i}+\psi_{i}\left(2 \mathrm{p}_{\tau}\right) .
\end{aligned}
$$

Proof of (4.29): the first two symmetries of $\psi_{i}$ in (4.36) imply that $\psi_{i}\left(2 \mathrm{p}_{\tau}^{+}\right)=$ $2 \psi_{i}\left(\mathrm{p}_{\tau}^{+}\right)$and $\psi_{i}\left(-2 \mathrm{p}_{\tau}^{-}\right)=2 \psi_{i}\left(-\mathrm{p}_{\tau}^{-}\right)$. The third symmetry of $(4.36)$ with $t=$ $-2 \mathrm{p}_{\tau}^{-}$implies that

$$
2 \widehat{\mathbf{p}}_{\tau}=p \psi_{1}\left(2 \mathrm{p}_{\tau}\right)=p\left(\psi_{1}\left(2 \mathbf{p}_{\tau}^{+}\right)-\psi_{1}\left(-2 \mathbf{p}_{\tau}^{-}\right)\right)=2 p\left(\psi_{1}\left(\mathbf{p}_{\tau}^{+}\right)-\psi_{1}\left(-\mathbf{p}_{\tau}^{-}\right)\right) .
$$

It remains to prove the last equality of (4.29). By the equalities on the previous line it suffices to prove that $\Psi\left(2 \mathrm{p}_{\tau}\right)=p \psi_{1}\left(2 \mathrm{p}_{\tau}\right)+q \psi_{2}\left(2 \mathrm{p}_{\tau}\right)=0$. Since $\tau>0, \alpha_{\tau} \in\left[-\frac{\pi}{2}, 0\right)$. Now since $\Psi(0)=0$ and $\sqrt{f(y)(t)}$ is continuous in $t$ and positive, (4.21b) implies that $\Psi(t)+\alpha_{\tau} \in(-\pi, 0)$ holds for all $t \in \mathbb{R}$. At 
The geometry of $\mathrm{SO}(p) \times \mathrm{SO}(q)$-invariant special Lagrangian cones 201

$t=2 \mathrm{p}_{\tau}$, we have $f(y)=f_{\max }=4 \tau_{\max }^{2}$ and $\dot{y}=-4 \tau_{\max } \cos \alpha_{\tau}$. Hence, (4.21a) and (4.21b) imply that $\mathrm{e}^{\mathrm{i}\left(\Psi+\alpha_{\tau}\right)}=\mathrm{e}^{\mathrm{i} \alpha_{\tau}}$ holds at $t=2 \mathrm{p}_{\tau}$. Hence $\Psi\left(2 \mathrm{p}_{\tau}\right)=0$, since $\Psi+\alpha_{\tau} \in(-\pi, 0)$.

Proof of (4.30): The symmetries of $\mathbf{w}_{\tau}$ claimed in (4.30) follow from (4.6), (4.29) and (4.36) and the expression (4.17) for $\mathbf{w}_{\tau}$ in terms of $y_{\tau}, \psi_{1}$ and $\psi_{2}$.

Extra symmetries for case $p=q$ : Define $\boldsymbol{z}: \mathbb{R} \rightarrow \mathbb{S}^{3}$ by $\boldsymbol{z}=\left(w_{2} \circ \mathbf{I}, w_{1} \circ \underline{\mathbf{T}}\right)$. Since $p=q$, we see that $\boldsymbol{z}$ also satisfies (3.8). Moreover, since $p=q$ the initial data $\mathbf{w}_{\tau}(0)$ (recall (3.24)) is invariant under exchange of $w_{1}$ and $w_{2}$, and therefore $\boldsymbol{z}(0)=\mathbf{w}_{\tau}(0)$. Hence by uniqueness of the initial value problem $\boldsymbol{z}$ coincides with $\mathbf{w}_{\tau}$ as claimed.

The first two symmetries of (4.32) follow from (4.31) and the relation between $w_{i}$ and $\psi_{i}$, given in (4.17). The final two symmetries of (4.32) follow from the first two and the existing symmetry $\psi_{i} \circ{\underline{\mathbf{p}_{\tau} / 2}}_{\mathbf{p}}=-\psi_{i}+\psi_{i}\left(\mathbf{p}_{\tau}\right)$ for $i=1,2$ (obtained from (4.36) using the fact that $\mathbf{p}_{\tau}^{+}=\frac{1}{2} \mathbf{p}_{\tau}$ ).

Periods and half-periods of $\mathbf{w}_{\boldsymbol{\tau}}$. To understand the extrinsic geometry of $X_{\tau}$ and in particular when $X_{\tau}$ factors through a closed embedding we need to understand when $\mathbf{w}_{\tau}$ gives rise to a closed curve in the space of isotropic $\mathrm{SO}(p) \times \mathrm{SO}(q)$ orbits (recall 3.3). To this end we define the periods and halfperiods of $\mathbf{w}_{\tau}$. The periods and half-periods of $\mathbf{w}_{\tau}$ control when the curve of isotropic orbits $\mathcal{O}_{\mathbf{w}_{\tau}}$ determined by $\mathbf{w}_{\tau}$ is a closed curve in the space of $\mathrm{SO}(p) \times \mathrm{SO}(q)$ orbits. As in 3.3 , for any $\mathbf{w} \in \mathbb{S}^{3}$ let $\mathcal{O}_{\mathbf{w}} \subset \mathbb{S}^{2(p+q)-1}$ denote the associated isotropic $\mathrm{SO}(p) \times \mathrm{SO}(q)$ orbit.

Definition 4.37. Fix a pair of admissible integers $p$ and $q$ and let $\mathbf{w}_{\tau}$ be any of the $(p, q)$-twisted SL curves defined in 3.23.

(i) We define the periods of $\mathbf{w}_{\tau}$ by

$$
\operatorname{Per}\left(\mathbf{w}_{\tau}\right):=\left\{x \in \mathbb{R} \mid \mathbf{w}_{\tau} \circ \mathbf{T}_{x}=\mathbf{w}_{\tau}\right\} .
$$

(ii) We define the half-periods of $\mathbf{w}_{\tau}$ by

$$
\operatorname{Per}_{\frac{1}{2}}\left(\mathbf{w}_{\tau}\right):=\left\{x \in \mathbb{R} \mid \mathcal{O}_{\mathbf{w}_{\tau} \circ \boldsymbol{T}_{x}(t)}=\mathcal{O}_{\mathbf{w}_{\tau}(t)} \forall t \in \mathbb{R}\right\},
$$

where as previously $\mathcal{O}_{\mathbf{w}} \subset \mathbb{S}^{2(p+q)-1}$ denotes the isotropic $\mathrm{SO}(p) \times$ $\mathrm{SO}(q)$ orbit associated with any point $\mathbf{w} \in \mathbb{S}^{3}$.

(iii) A strict half-period is any half-period which is not a period of $\mathbf{w}_{\tau}$.

In other words, $x$ is a half-period of $\mathbf{w}_{\tau}$ if $\mathbf{w}_{\tau} \circ \mathrm{T}_{x}$ and $\mathbf{w}_{\tau}$ give rise to the same parametrized curve of isotropic $\mathrm{SO}(p) \times \mathrm{SO}(q)$-orbits in $\mathbb{S}^{2(p+q)-1}$. 
Using 3.3 we see that $x$ is a half-period of $\mathbf{w}_{\tau}$ if and only if

$$
\mathbf{w}_{\tau} \circ \mathrm{T}_{x}=\rho_{j k} \circ \mathbf{w}_{\tau} \quad \text { for some } \rho_{j k} \in \operatorname{Stab}_{p, q}
$$

where $\mathrm{Stab}_{p, q}$ is the finite subgroup of $\mathrm{U}(2)$ defined by

$$
\operatorname{Stab}_{p, q}= \begin{cases}\left(\begin{array}{cc} 
\pm 1 & 0 \\
0 & \pm 1
\end{array}\right) \cong \mathbb{Z}_{2} \times \mathbb{Z}_{2} & \text { if } p>1 \\
\left(\begin{array}{cc}
1 & 0 \\
0 & \pm 1
\end{array}\right) \cong \mathbb{Z}_{2} & \text { if } p=1\end{cases}
$$

More explicitly, we have

$$
\begin{gathered}
\operatorname{Per}_{\frac{1}{2}}\left(\mathbf{w}_{\tau}\right):=\left\{x \in \mathbb{R} \mid \exists(j, k) \in\langle(+, \pm)\rangle \leq \mathbb{Z}_{2} \times \mathbb{Z}_{2}\right. \text { such that } \\
\left.\rho_{j k} \circ \mathbf{w}_{\tau}=\mathbf{w}_{\tau} \circ \mathrm{T}_{x}\right\}, \quad \text { if } p=1 ;
\end{gathered}
$$

or

$$
\begin{aligned}
\operatorname{Per}_{\frac{1}{2}}\left(\mathbf{w}_{\tau}\right):= & \left\{x \in \mathbb{R} \mid \exists(j, k) \in \mathbb{Z}_{2} \times \mathbb{Z}_{2} \text { such that } \rho_{j k} \circ \mathbf{w}_{\tau}=\mathbf{w}_{\tau} \circ \mathrm{T}_{x}\right\}, \\
& \text { if } p>1 .
\end{aligned}
$$

If $x$ satisfies (4.40) for $(j, k) \in \mathbb{Z}_{2} \times \mathbb{Z}_{2}$ then we call $x$ a half-period of $\mathbf{w}_{\tau}$ of type $(j k)$. We see immediately from (4.40) that $2 \operatorname{Per}_{\frac{1}{2}}\left(\mathbf{w}_{\tau}\right) \subset \operatorname{Per}\left(\mathbf{w}_{\tau}\right)$; this explains the terminology half-period.

The importance of the half-periods of $\mathbf{w}_{\tau}$ is explained by the following

Proposition 4.44. Suppose $0<|\tau|<\tau_{\max }$ and let $X_{\tau}$ be one of the $S O(p) \times$ $S O(q)$-invariant special Legendrian cylinders defined in (3.29). Suppose there exist triples $\left(t_{1}, \sigma_{1}, \sigma_{2}\right),\left(t_{2}, \sigma_{1}^{\prime}, \sigma_{2}^{\prime}\right) \in \mathrm{Cyl}^{p, q}$ such that

$$
X_{\tau}\left(t_{1}, \sigma_{1}, \sigma_{2}\right)=X_{\tau}\left(t_{2}, \sigma_{1}^{\prime}, \sigma_{2}^{\prime}\right) .
$$

Then $t_{2}-t_{1} \in \operatorname{Per}_{\frac{1}{2}}\left(\mathbf{w}_{\tau}\right)$. Moreover, if $t_{2}-t_{1} \in \operatorname{Per}\left(\mathbf{w}_{\tau}\right)$ then $\sigma_{1}=\sigma_{1}^{\prime}$ and $\sigma_{2}=\sigma_{2}^{\prime}$.

Proof. See [13, Prop 6.32].

For completeness here is the analogue of 4.44 for the case $\tau=\tau_{\max }$. 
The geometry of $\mathrm{SO}(p) \times \mathrm{SO}(q)$-invariant special Lagrangian cones 203

Lemma 4.46. Let $X_{\tau}: \mathrm{Cyl}^{p, q} \rightarrow \mathbb{S}^{2(p+q)-1}$ be the $S O(p) \times S O(q)$-equivariant special Legendrian immersion defined in (3.29), with $\tau=\tau_{\text {max }}$. Then there exists a pair of triples $\left(t_{1}, \sigma_{1}, \sigma_{2}\right),\left(t_{2}, \sigma_{1}^{\prime}, \sigma_{2}^{\prime}\right) \in \mathbb{R} \times \mathbb{S}^{p-1} \times \mathbb{S}^{q-1}$ such that

$$
X_{\tau}\left(t_{1}, \sigma_{1}, \sigma_{2}\right)=X_{\tau}\left(t_{2}, \sigma_{1}^{\prime}, \sigma_{2}^{\prime}\right) \text {, }
$$

if and only if

$$
t_{2}-t_{1}=\frac{\operatorname{lcm}(p, q) \pi}{n \tau_{\max }} l, \quad \sigma_{1}=(-1)^{j l} \sigma_{1}^{\prime}, \quad \sigma_{2}=(-1)^{k l} \sigma_{2}^{\prime}, \quad \text { for any } l \in \mathbb{Z},
$$

where $j=q / \operatorname{hcf}(p, q)$ and $k=p / \operatorname{hcf}(p, q)$.

$$
\operatorname{Per}\left(X_{\tau}\right)=\left\langle\mathrm{T}_{x} \circ\left((-1)^{j} \operatorname{Id}_{\mathbb{S}^{p-1}},(-1)^{k} \operatorname{Id}_{\mathbb{S}^{q-1}}\right)\right\rangle \quad \text { where } x=\frac{\operatorname{lcm}(p, q) \pi}{n \tau_{\max }} .
$$

Proof. This is a straightforward computation using the explicit expression for $\mathbf{w}_{\tau}$ (see 3.31.v).

We now completely determine the periods and half-periods of $\mathbf{w}_{\tau}$. We see from (4.27) and (4.30) that $\hat{\mathrm{T}}_{2 \widehat{\mathrm{p}}_{\tau}} \in \mathrm{U}(2)$ (recall $\hat{\mathrm{T}}_{x} \in \mathrm{U}(2)$ is defined in (3.9) and $\widehat{p}_{\tau}$ is defined by (4.23)) plays a fundamental role in the geometry of $\mathbf{w}_{\tau}$. We call $\hat{\mathrm{T}}_{2 \widehat{\mathrm{p}}_{\tau}}$ the rotational period of $\mathbf{w}_{\tau}$, since by (4.27) and (4.30) $\hat{\mathrm{T}}_{2 \widehat{\mathrm{p}}_{\tau}}$ controls how $\mathbf{w}_{\tau}$ gets rotated as we move from one domain of periodicity of $y_{\tau}$ to the next. This motivates the following definition.

Definition 4.48. Define $k_{0} \in \mathbb{N} \cup\{+\infty\}$ to be the order of the rotational period $\hat{\mathrm{T}}_{2 \widehat{\mathrm{p}}_{\tau}} \in \mathrm{U}(2)$. We set $k_{0}=+\infty$ if the rotational period has infinite order.

For the rest of this section we always assume $0<|\tau|<\tau_{\max }$ unless stated otherwise. We can completely describe the period lattice $\operatorname{Per}\left(\mathbf{w}_{\tau}\right)$ and the half-period lattice $\operatorname{Per}_{\frac{1}{2}}\left(\mathbf{w}_{\tau}\right)$ in terms of $k_{0}$ :

Lemma 4.49. Fix a pair of admissible integers $p$ and $q$ and let $n=p+q$. $k_{0}$ the order of the rotational period $\hat{\mathrm{T}}_{2 \widehat{\mathrm{p}}_{\tau}}$ defined in 4.48 can also be characterized as

$$
k_{0}=\min \left\{k \in \mathbb{Z}^{+} \mid k \widehat{\mathrm{p}}_{\tau} \in \pi \operatorname{lcm}(p, q) \mathbb{Z}\right\},
$$

and the following are equivalent

$$
\widehat{\mathrm{p}}_{\tau} \notin \pi \mathbb{Q} \quad \Longleftrightarrow \quad k_{0}=\infty \quad \Longleftrightarrow \quad \operatorname{Per}_{\frac{1}{2}}\left(\mathbf{w}_{\tau}\right)=\operatorname{Per}\left(\mathbf{w}_{\tau}\right)=(0) .
$$


If $\widehat{\mathrm{p}}_{\tau} \in \pi \mathbb{Q}$, then in all cases we have

$$
\operatorname{Per}\left(\mathbf{w}_{\tau}\right)=2 k_{0} \mathbf{p}_{\tau} \mathbb{Z}
$$

(i) if $k_{0}$ is odd then $\operatorname{Per}_{\frac{1}{2}}\left(\mathbf{w}_{\tau}\right)=\operatorname{Per}\left(\mathbf{w}_{\tau}\right)=2 k_{0} \mathbf{p}_{\tau} \mathbb{Z}$, i.e., $\mathbf{w}_{\tau}$ has no strict half-periods.

(ii) if $k_{0}$ is even and $p>1$ then $\operatorname{Per}_{\frac{1}{2}}\left(\mathbf{w}_{\tau}\right)=\frac{1}{2} \operatorname{Per}\left(\mathbf{w}_{\tau}\right)=k_{0} \mathbf{p}_{\tau} \mathbb{Z}$. Moreover, for fixed $p$ and $q$ every strict half-period of $\mathbf{w}_{\tau}$ is of type $(j k)$ where $j=q / \operatorname{hcf}(p, q) \bmod 2$ and $k=p / \operatorname{hcf}(p, q) \bmod 2$.

(iii) (a) if $k_{0}$ is even, $p=1$ and $n$ is even then $\operatorname{Per}_{\frac{1}{2}}\left(\mathbf{w}_{\tau}\right)=\operatorname{Per}\left(\mathbf{w}_{\tau}\right)=$ $2 k_{0} \mathrm{p}_{\tau} \mathbb{Z}$.

(b) if $k_{0}$ is even, $p=1$ and $n$ is odd then $\operatorname{Per}_{\frac{1}{2}}\left(\mathbf{w}_{\tau}\right)=\frac{1}{2} \operatorname{Per}\left(\mathbf{w}_{\tau}\right)=$ $k_{0} \mathrm{p}_{\tau} \mathbb{Z}$.

Proof. See [13, Lemma 6.39].

Remark 4.52. For $\tau=0, X_{0}$ is an embedding whose image is contained in the standard totally real equatorial sphere $\mathbb{S}^{n-1} \subset \mathbb{R}^{n} \subset \mathbb{C}^{n}$. In this case $\operatorname{Per}\left(\mathbf{w}_{\tau}\right)=\operatorname{Per}_{\frac{1}{1}}\left(\mathbf{w}_{\tau}\right)=(0)$. For $\tau=\tau_{\max }$, we leave it as an elementary exercise for the reader to use the explicit expression given in 3.31 to write down the period and half-period lattices of $\mathbf{w}_{\tau}$ in this case (see also Proposition 4.46).

By combining Proposition 4.44 with our results on $\operatorname{Per}\left(\mathbf{w}_{\tau}\right)$ and $\operatorname{Per}_{\frac{1}{2}}\left(\mathbf{w}_{\tau}\right)$ in 4.49 we have a complete understanding of the self-intersection points of $X_{\tau}$; see also 7.44.iii.

\section{Discrete symmetries of $\boldsymbol{X}_{\tau}$}

In addition to its intrinsic interest, the full group of symmetries of our $\mathrm{SO}(p) \times \mathrm{SO}(q)$-equivariant building blocks $X_{\tau}$ plays a fundamental role in our subsequent gluing constructions $[9,10]$. These additional discrete symmetries that the $X_{\tau}$ possess allow us to impose certain symmetries throughout our entire gluing construction (see the discussion in our survey paper [12].) The imposition of these symmetries simplifies some aspects of the gluing construction.

Fix a pair of admissible integers $p$ and $q$ and set $n=p+q$. Recall from 2.6 that we defined a symmetry of $X_{\tau}$ to be a pair $(\widetilde{\mathrm{M}}, \mathrm{M}) \in \mathrm{O}(2 n) \times$ 
The geometry of $\mathrm{SO}(p) \times \mathrm{SO}(q)$-invariant special Lagrangian cones 205

$\operatorname{Diff}\left(\mathrm{Cyl}^{p, q}\right)$ such that

$$
\widetilde{\mathrm{M}} \circ X_{\tau}=X_{\tau} \circ \mathrm{M}
$$

where Diff $\left(\mathrm{Cyl}^{p, q}\right)$ denotes the group of diffeomorphisms of the domain of $X_{\tau}$. Recall from 2.11 that for any symmetry $(\widetilde{\mathrm{M}}, \mathrm{M})$ then $\mathrm{M}$ is an isometry of the pullback metric $g_{\tau}:=X_{\tau}^{*} g_{\mathbb{S}^{2 n-1}}$. This motivates the next subsection where we determine the structure of Isom $\left(\mathrm{Cyl}^{p, q}, g_{\tau}\right)$ completely. In the following subsection we will show that in fact $\operatorname{Sym}\left(X_{\tau}\right) \cong \operatorname{Isom}\left(\mathrm{Cyl}^{p, q}, g_{\tau}\right)$, i.e., that every isometry of $g_{\tau}$ gives rise to a symmetry of $X_{\tau}$. We also determine the structure of the group $\widehat{\operatorname{Sym}}\left(X_{\tau}\right) \subset \mathrm{O}(2 n)$.

Symmetries of the pullback metric $\boldsymbol{g}_{\boldsymbol{\tau}}$. In this subsection we study $\operatorname{Isom}\left(\mathrm{Cyl}^{p, q}, g_{\tau}\right)$ the group of isometries of the pullback metric $g_{\tau}:=X_{\tau}^{*} g_{\mathbb{S}^{2 n-1}}$ on the cylinder $\mathrm{Cyl}^{p, q}$. In other words we study the symmetries of the intrinsic geometry of $X_{\tau}$. Recall from 3.30.ii that the pullback metric $g_{\tau}$ on $\mathrm{Cyl}^{p, q}$ depends only on the function $y_{\tau}$; isometries of $g_{\tau}$ are thus intimately connected with the symmetries of $y_{\tau}$ studied in 4.3 . The reader should recall the elementary group theory reviewed in Section 2 which is assumed throughout the rest of this section and in Section 6 .

We begin by establishing notation. Any element $T \in \operatorname{Isom}(\mathbb{R})$ acts as an element of $\operatorname{Diff}\left(\mathrm{Cyl}^{p, q}\right)$ by

$$
\left(t, \sigma_{1}, \sigma_{2}\right) \mapsto\left(\mathrm{T} t, \sigma_{1}, \sigma_{2}\right)
$$

If $p>1$ any $\mathrm{O}=\left(\mathrm{O}_{1}, \mathrm{O}_{2}\right) \in \mathrm{O}(p) \times \mathrm{O}(q)$ acts as an element of $\operatorname{Diff}\left(\mathrm{Cyl}^{p, q}\right)$ by

$$
\left(t, \sigma_{1}, \sigma_{2}\right) \mapsto\left(t, \mathrm{O}_{1} \sigma_{1}, \mathrm{O}_{2} \sigma_{2}\right)
$$

Similarly, any element of $\mathrm{O} \in \mathrm{O}(n-1)$ acts as an element of Diff( $\left.\mathrm{Cyl}^{1, n-1}\right)$ by

$$
(t, \sigma) \mapsto(t, \mathrm{O} \sigma)
$$

We also define the exchange map $\mathrm{E} \in \operatorname{Diff}\left(\mathrm{Cyl}^{p, p}\right)$ by

$$
\mathrm{E}\left(t, \sigma_{1}, \sigma_{2}\right)=\left(t, \sigma_{2}, \sigma_{1}\right)
$$

Conjugation by the exchange map $\mathrm{E}$ defined in (5.4) defines an involution $\mathrm{E}^{\prime} \in \operatorname{Aut} \operatorname{Diff}\left(\mathrm{Cyl}^{p, p}\right)$. $\mathrm{E}^{\prime}$ leaves the subgroup $\mathrm{O}(p) \times \mathrm{O}(p) \subset \operatorname{Diff}\left(\mathrm{Cyl}^{p, p}\right)$ 
invariant and on it acts by

$$
\mathrm{E}^{\prime}\left(\mathrm{O}_{1}, \mathrm{O}_{2}\right)=\left(\mathrm{O}_{2}, \mathrm{O}_{1}\right)
$$

There is an obvious isomorphism $\rho_{E}:\langle E\rangle \rightarrow\left\langle\mathrm{E}^{\prime}\right\rangle \subset \operatorname{Aut} \mathrm{O}(p) \times \mathrm{O}(p) \subset$ Aut Diff $\left(\mathrm{Cyl}^{p, p}\right)$ given by

$$
\mathrm{E} \mapsto \mathrm{E}^{\prime}
$$

(5.5) implies that $\langle\mathrm{E}\rangle \cdot(\mathrm{O}(p) \times \mathrm{O}(p))=(\mathrm{O}(p) \times \mathrm{O}(p)) \cdot\langle\mathrm{E}\rangle$ and hence by the group theory discussion in Section 2 the set $\langle\mathrm{E}\rangle \cdot(\mathrm{O}(p) \times \mathrm{O}(p)) \subset$ $\operatorname{Isom}\left(\mathbb{S}^{p-1} \times \mathbb{S}^{p-1}\right) \subset \operatorname{Diff}\left(\mathrm{Cyl}^{p, p}\right)$ forms a group $G$. Moreover $\mathrm{O}(p) \times \mathrm{O}(p)$ is a normal subgroup of $G$ and clearly $\mathrm{O}(p) \times \mathrm{O}(p) \cap\langle\mathrm{E}\rangle=(\mathrm{Id})$. Hence $G$ is the semidirect product of $\mathrm{O}(p) \times \mathrm{O}(p)$ by $\langle\mathrm{E}\rangle \cong \mathbb{Z}_{2}$ where the twisting homomorphism $\rho:\langle\mathrm{E}\rangle \rightarrow \operatorname{Aut} \mathrm{O}(p) \times \mathrm{O}(p)$ is $\rho_{E}$ defined above, i.e., $G=$ $(\mathrm{O}(p) \times \mathrm{O}(p)) \rtimes_{\rho_{E}}\langle\mathrm{E}\rangle$. (In fact, $G \cong \operatorname{Isom}\left(\mathbb{S}_{r}^{p-1} \times \mathbb{S}_{r}^{p-1}\right)$; see (5.13) below.)

The main result of this section is the following:

Proposition 5.6 (Isometries of the pullback metric on $\mathrm{Cyl}^{p, q}$ ). Let $g_{\tau}:=X_{\tau}^{*} g_{\mathbb{S}^{2 n-1}}$ denote the metric induced on $\mathrm{Cyl}^{p, q}$ by the immersion $X_{\tau}$ : $\mathrm{Cyl}^{p, q} \rightarrow \mathbb{S}^{2(p+q)-1}$. For $0<|\tau|<\tau_{\max }$,

$$
\operatorname{Isom}\left(\mathrm{Cyl}^{p, q}, g_{\tau}\right)=\mathbf{D} \cdot O
$$

where

(i) for $p=1, \mathbf{D}=\left\langle\underline{\mathrm{T}}, \mathrm{T}_{2 \mathrm{p}_{\tau}}\right\rangle$ and $O=O(n-1)$.

(ii) for $p>1$ and $p \neq q, \mathbf{D}=\left\langle\underline{\mathbf{T}}_{\mathbf{p}_{\tau}^{+}}, \underline{\mathbf{T}}_{-\mathbf{p}_{\tau}^{-}}\right\rangle$and $O=O(p) \times O(q)$.

(iii) for $p>1$ and $p=q, \mathbf{D}=\left\langle\underline{\mathrm{T}} \circ \mathbf{E}, \underline{\mathbf{T}}_{\mathbf{p}_{\tau} / 2}\right\rangle$ and $O=O(p) \times O(p)$.

Hence we have

$$
\begin{aligned}
& \operatorname{Isom}\left(\mathrm{Cyl}^{p, q}, g_{\tau}\right) \\
& \quad= \begin{cases}\left\langle\underline{\mathbf{T}}, \mathbf{T}_{2 \mathbf{p}_{\tau}}\right\rangle \times O(n-1) \cong \mathbf{D}_{\infty} \times O(n-1), & \text { for } p=1 ; \\
\left\langle\underline{\mathbf{T}}_{\mathbf{p}_{\tau}^{+}}, \underline{\mathbf{T}}_{\mathbf{p}_{\tau}}\right\rangle \times O(p) \times O(q) \cong \mathbf{D}_{\infty} \times O(p) \times O(q) & \text { for } p>1 \text { and } p \neq q ; \\
\left\langle\underline{\mathbf{I}} \circ \mathbf{E}, \underline{\mathbf{T}}_{\mathbf{p}_{\tau} / 2}\right\rangle \times O(p) \times O(p) \cong(O(p) \times O(p)) \rtimes_{\rho} \mathbf{D}_{\infty} & \text { for } p>1 \text { and } p=q ;\end{cases}
\end{aligned}
$$

where the twisting homomorphism

$$
\rho:\left\langle\underline{\mathrm{T}} \circ \mathbf{E}, \underline{\mathrm{T}}_{\mathbf{p}_{\tau} / 2}\right\rangle \cong \mathbf{D}_{\infty} \rightarrow \text { Aut } O(p) \times O(p)
$$

is defined by $\rho(\gamma)= \begin{cases}\mathrm{Id} & \text { if } \gamma \text { is a word containing an even number of copies of } \mathrm{T} \circ \mathrm{E}, \\ \mathrm{E}^{\prime} & \text { if } \gamma \text { is a word containing an odd number of copies of } \mathrm{I} \circ \mathrm{E},\end{cases}$ where $\mathrm{E}^{\prime}$ is the involution defined in (5.5). 
The geometry of $\mathrm{SO}(p) \times \mathrm{SO}(q)$-invariant special Lagrangian cones 207

Remark 5.7. When $p=q$ the subgroup $\mathbf{D}^{\prime} \subset \mathbf{D}$ generated by the two elements $\underline{T}_{p_{\tau} / 2}$ and $\underline{T}_{-p_{\tau} / 2}=(\underline{T} \circ E) \circ \underline{T}_{p_{\tau} / 2} \circ(\underline{T} \circ E)$ is also isomorphic to the infinite dihedral group $\mathbf{D}_{\infty}$, and corresponds to the symmetries that are shared with the case $p \neq q$. Alternatively, $\mathbf{D}^{\prime} \subset \mathbf{D}$ is the subgroup consisting of all words containing an even number of copies of $\mathbf{T} \circ \mathbf{E}$ and hence $\mathbf{D}^{\prime}$ is the kernel ker $\rho$ of the twisting homomorphism $\rho: \mathbf{D} \rightarrow$ Aut $\mathrm{O}(p) \times \mathrm{O}(p)$. Hence $\operatorname{Isom}\left(\mathrm{Cyl}^{p, p}\right)$ contains a subgroup isomorphic to $(\mathrm{O}(p) \times \mathrm{O}(p)) \times \mathbf{D}^{\prime} \cong \mathrm{O}(p)$ $\times \mathrm{O}(p) \times \mathbf{D}_{\infty}$.

Proof. Recall from 3.30 that $g_{\tau}$ can be written in terms of $y_{\tau}$ as

$$
g_{\tau}= \begin{cases}y_{\tau}^{q-1}\left(1-y_{\tau}\right)^{p-1} d t^{2}+\left(1-y_{\tau}\right) g_{\mathbb{S}^{p-1}}+y_{\tau} g_{\mathbb{S}^{q-1}}, & \text { for } p>1 \\ y_{\tau}^{n-2} d t^{2}+y_{\tau} g_{\mathbb{S}^{n-2}} & \text { for } p=1\end{cases}
$$

It follows immediately from (5.8) that for $p>1$ any element of $\mathrm{O}(p) \times \mathrm{O}(q)$ acting as in (5.2) is an isometry of $g_{\tau}$. Similarly, for $p=1$ we have $\mathrm{O}(n-1) \subset$ $\operatorname{Isom}\left(\mathrm{Cyl}^{p, q}, g_{\tau}\right)$. For any $\mathbf{S} \in \operatorname{Isom}(\mathbb{R})$ satisfying $y_{\tau} \circ \mathbf{S}=y_{\tau}$, extend $\mathbf{S}$ to a diffeomorphism of $\mathrm{Cyl}^{p, q}$ as described in (5.1). Since $\mathrm{S}$ preserves $y_{\tau}$ it follows from (5.8) that $\mathrm{S} \in \operatorname{Isom}\left(\mathrm{Cyl}^{p, q}, g_{\tau}\right)$. Recall from (4.7) that in the special case $p=q, y_{\tau}$ possesses an additional symmetry $\mathbf{I}$ sending $y_{\tau} \mapsto 1-y_{\tau}$. Because of this symmetry and the form of (5.8) the map $\mathrm{I} \circ \mathrm{E} \in \operatorname{Diff}\left(\mathrm{Cyl}{ }^{p, p}\right)$ defined by $\left(t, \sigma_{1}, \sigma_{2}\right) \mapsto\left(-t, \sigma_{2}, \sigma_{1}\right)$ also belongs to $\operatorname{Isom}\left(\mathrm{Cyl}^{p, p}, g_{\tau}\right)$. By using the symmetries of $y_{\tau}$ established in 4.3 it follows that $\mathbf{D}$ forms a subgroup of $\operatorname{Isom}\left(\mathrm{Cyl}^{p, q}, g_{\tau}\right)$ where $\mathbf{D}$ is the discrete group defined for the three cases (i) $p=1$, (ii) $p>1, p \neq q$, (iii) $p>1, p=q$ in the statements 5.6.i-iii, respectively. Hence we have established that $\mathbf{D} \cdot \mathrm{O} \subseteq \operatorname{Isom}\left(\mathrm{Cyl}^{p, q}\right)$ where $\mathrm{O}=\mathrm{O}(p) \times \mathrm{O}(q)$ if $p>1$ and $\mathrm{O}=\mathrm{O}(n-1)$ if $p=1$.

It remains to prove that any element in Isom $\left(\mathrm{Cyl}^{p, q}, g_{\tau}\right)$ belongs to $\mathbf{D} \cdot \mathrm{O}$. We begin by introducing some useful terminology. A meridian of $\mathrm{Cyl}^{p, q}$ is any hypersurface of the form $\{t\} \times \operatorname{Mer}^{p, q}$ (recall (3.2)) for any fixed $t \in$ $\mathbb{R}$. Let $\Pi: \mathrm{Cyl}^{p, q} \rightarrow \mathrm{Mer}^{p, q}$ denote projection $(t, \sigma) \mapsto \sigma$. The generator of $\mathrm{Cyl}^{p, q}$ through the point $\sigma \in \mathrm{Mer}^{p, q}$ is the curve $\gamma_{\sigma}: \mathbb{R} \rightarrow \mathrm{Cyl}^{p, q}$ given by

$$
t \mapsto(t, \sigma),
$$

i.e., a generator is a curve $\gamma_{\sigma}$ whose projection $\Pi \circ \gamma_{\sigma}$ to the cross section Mer $^{p, q}$ is the constant map $\sigma: \mathbb{R} \rightarrow$ Mer $^{p, q}$. Suitably parametrized any generator is a minimizing geodesic, i.e., it minimizes the $g_{\tau}$-distance between any two points on its image. Note that the meridians can be characterized as the integral manifolds of the distribution $\mathcal{D}=\left\langle\partial_{t}\right\rangle^{\perp}$ of hyperplanes normal to the tangent lines to the generators of $\mathrm{Cyl}^{p, q}$. 
The key to proving $\operatorname{Isom}\left(\mathrm{Cyl}^{p, q}, g_{\tau}\right)=\mathbf{D} \cdot \mathrm{O}$ is to establish that any $\mathrm{I} \in \operatorname{Isom}\left(\mathrm{Cyl}^{p, q}, g_{\tau}\right)$ maps meridians to meridians. It suffices to prove that any minimizing geodesic of $g_{\tau}$ must be a generator, since then any isometry must map generators to generators, preserve the hyperplane distribution $\mathcal{D}$ and therefore map meridians to meridians. To prove that any minimizing geodesic is a generator we will make use of some special isometries of Isom $\left(\mathrm{Cyl}^{p, q}, g_{\tau}\right)$ which we now describe.

If $t_{c}$ is any critical point of $y_{\tau}$ then reflection $\underline{\mathrm{T}}_{t_{c}} \in \operatorname{Diff}\left(\mathrm{Cyl}{ }^{p, q}\right)$ across the meridian $\left\{t_{c}\right\} \times \operatorname{Mer}^{p, q}$ is contained in the group $\mathbf{D}$ and hence by the first part of the proposition is an isometry of $\operatorname{Isom}\left(\mathrm{Cyl}^{p, q}, g_{\tau}\right)$. For $p=1$ and any $\sigma \in \mathbb{S}^{n-2}$ we denote by $\mathrm{R}_{\sigma} \in \mathrm{O}(n-1)$ reflection with respect to the line through $\sigma$ in $\mathbb{R}^{n-1}$. For $p>1$ and $\sigma=\left(\sigma^{\prime}, \sigma^{\prime \prime}\right) \in \mathbb{S}^{p-1} \times \mathbb{S}^{q-1}$ we define $\mathrm{R}_{\sigma}:=$ $\left(\mathrm{R}_{\sigma^{\prime}}, \mathrm{R}_{\sigma^{\prime \prime}}\right) \in \mathrm{O}(p) \times \mathrm{O}(q)$ where $\mathrm{R}_{\sigma^{\prime}} \in \mathrm{O}(p)$ and $\mathrm{R}_{\sigma^{\prime \prime}} \in \mathrm{O}(q)$ denote reflections in the line through $\sigma^{\prime}$ in $\mathbb{R}^{p}$ and the line through $\sigma^{\prime \prime}$ in $\mathbb{R}^{q}$ respectively. By the first part of the proposition $\underline{\mathrm{T}}_{t_{c}} \circ \mathrm{R}_{\sigma} \in \mathbf{D} \cdot \mathrm{O} \subset \operatorname{Isom}\left(\mathrm{Cyl}{ }^{p, q}, g_{\tau}\right)$.

The key properties of the isometry $\underline{\mathrm{T}}_{t_{c}} \circ \mathrm{R}_{\sigma}$ are that it fixes the point $\left(t_{c}, \sigma\right) \in\left\{t_{c}\right\} \times \mathrm{Mer}^{p, q}$ and acts by $-\mathrm{Id}$ on the tangent space $T_{\left(t_{c}, \sigma\right)} \mathrm{Cyl}^{p, q}$. Therefore $\underline{\mathrm{T}}_{t_{c}} \circ \mathrm{R}_{\sigma}$ sends any geodesic $\gamma$ passing through $\left(t_{c}, \sigma\right)$ to another geodesic passing through $\left(t_{c}, \sigma\right)$ whose tangent vector at this point is the negative of the tangent vector of the initial geodesic. Hence uniqueness of the initial value problem for geodesics implies the following symmetry of $\gamma$ :

$$
\gamma \circ \underline{\mathrm{T}}_{s}=\underline{\mathrm{T}}_{t_{c}} \circ \mathrm{R}_{\sigma} \circ \gamma, \quad \text { where } \gamma(s)=\left(t_{c}, \sigma\right)
$$

Let $2 d$ denote the distance between the boundary meridians of any domain of periodicity of $g_{\tau}$. Equivalently, $d$ is the distance between two consecutive critical meridians (a meridian of the form $\left\{t_{k}\right\} \times \operatorname{Mer}^{p, q}$ for some critical point $t_{k}$ of $\left.y_{\tau}\right) . d$ is realized along any generator and any other curve connecting two such meridians has strictly greater length. (Also $2 d$ depends smoothly on $\tau$ and tends to $\pi$, the diameter of the unit sphere $\mathbb{S}^{p+q-1}$ as $\tau \rightarrow 0$ ).

Suppose $\gamma: \mathbb{R} \rightarrow \mathrm{Cyl}^{p, q}$ is a geodesic parametrized by arc-length which is minimizing. By using the obvious piecewise smooth comparison curve, we see that the diameter of $k$ consecutive domains of periodicity of $g_{\tau}$ is bounded above by $2 k d+D$ where $D$ is the largest diameter of any meridian in a domain of periodicity of $g_{\tau}$. Since $\gamma$ is a minimizing geodesic the diameter of its image is infinite, and therefore $\gamma$ intersects every meridian $\{t\} \times \operatorname{Mer}^{p, q}$. $y_{\tau}$ is non-constant and $2 \mathrm{p}_{\tau}$-periodic and therefore has countably infinitely many critical points $t_{c}$ that we label by the strictly increasing sequence 
The geometry of $\mathrm{SO}(p) \times \mathrm{SO}(q)$-invariant special Lagrangian cones 209

$\left(t_{k}\right)_{k \in \mathbb{Z}}$. By Lemma 4.3 and (4.10) the sequence $\left(t_{k}\right)$ satisfies

$$
t_{k}-t_{l}=(k-l) \mathrm{p}_{\tau}, \quad \text { for any } k>l .
$$

(If $p=1$ we could normalize so that $t_{0}=0$ and hence $t_{k}=k \mathrm{p}_{\tau}$. If $p>1$ we could normalize so that $t_{0}=\mathrm{p}_{\tau}^{+}$and therefore $t_{-1}=-\mathrm{p}_{\tau}^{-}$.) Since $\gamma$ intersects every meridian, there exists an increasing sequence $\left(s_{k}\right)_{k \in \mathbb{Z}}$ and a unique sequence of points $\sigma_{k} \in \mathrm{Mer}^{p, q}$ so that

$$
\gamma\left(s_{k}\right)=\left(t_{k}, \sigma_{k}\right)
$$

In other words, $s_{k}$ is the arc-length parameter at which the minimizing geodesic $\gamma$ intersects the $k$ th critical meridian $\left\{t_{k}\right\} \times \mathrm{Mer}^{p, q}$. By time-translation invariance of geodesics without loss of generality we may assume that $s_{0}=0$. Applying the isometry $\underline{\mathbf{T}}_{t_{k}} \circ \mathrm{R}_{\sigma_{k}}$ as in (5.9) we deduce that the minimizing geodesic $\gamma$ has the symmetries

$$
\gamma \circ \underline{I}_{s_{k}}=\underline{\mathbf{I}}_{t_{k}} \circ \mathrm{R}_{\sigma_{k}} \circ \gamma, \quad \text { for any } k \in \mathbb{Z},
$$

for the sequence $\left(s_{k}\right)$ defined above in (5.10). Composing the two reflectional symmetries arising from (5.11) by setting $k=0$ and $k=1$ and using (4.14) together with the fact that $\underline{\mathrm{I}}, \mathrm{T}_{x} \in \operatorname{Diff}\left(\mathrm{Cyl}^{p, q}\right)$ commute with $\mathrm{O}(p) \times \mathrm{O}(q)$ for $p>1$ (respectively with $\mathrm{O}(n-1)$ for $p=1$ ) we obtain

$$
\gamma \circ \mathrm{T}_{2 s_{1}}=\mathrm{T}_{2\left(t_{1}-t_{0}\right)} \circ \mathrm{R}_{\sigma_{1}} \circ \mathrm{R}_{\sigma_{0}} \circ \gamma=\mathrm{T}_{2 \mathrm{p}_{\tau}} \circ \mathrm{R}_{\sigma_{1}} \circ \mathrm{R}_{\sigma_{0}} \circ \gamma .
$$

By iteration of (5.12) we have

$$
\begin{gathered}
\gamma \circ \mathrm{T}_{2 k s_{1}=}=\left(\mathrm{T}_{2 \mathrm{p}_{\tau}} \circ \mathrm{R}_{\sigma_{1}} \circ \mathrm{R}_{\sigma_{0}}\right)^{k} \circ \gamma=\mathrm{T}_{2 k \mathrm{p}_{\tau}} \circ\left(\mathrm{R}_{\sigma_{1}} \circ \mathrm{R}_{\sigma_{0}}\right)^{k} \circ \gamma, \\
\text { for any } k \in \mathbb{N}
\end{gathered}
$$

and hence that

$$
\gamma\left(2 k s_{1}\right) \in\left\{2 k \mathrm{p}_{\tau}+t_{0}\right\} \times \operatorname{Mer}^{p, q}=\left\{t_{2 k}\right\} \times \operatorname{Mer}^{p, q} .
$$

It follows from the definition of $s_{k}$ given in (5.10) that $s_{2 k}=2 k s_{1}$. Therefore since $\gamma$ is a minimizing geodesic parametrized by arc-length we have

$$
\operatorname{dist}\left(\gamma\left(s_{2 k}\right), \gamma(0)\right)=s_{2 k}=2 k s_{1}, \quad \text { for all } k \in \mathbb{N} .
$$


On the other hand, by our previous (crude) diameter bound for the union of any $k$ consecutive domains of periodicity of $g_{\tau}$ we have

$$
2 k s_{1} \leq 2 k d+D, \quad \text { for any } k \in \mathbb{N},
$$

where as previously $D$ denotes the largest diameter of any meridian and $d$ is the distance between two consecutive critical meridians (which as we have already stated is attained only by generators). Dividing by $k$ and taking $k \rightarrow \infty$ we conclude that $s_{1} \leq d$ and hence that $\gamma$ is a generator.

It remains to use the fact that any $\mathrm{I} \in \operatorname{Isom}\left(\mathrm{Cyl}^{p, q}, g_{\tau}\right)$ maps meridians to meridians to prove that $\mathbf{I} \in \mathbf{D} \cdot \mathrm{O}$. For the case $p>1$ we will need the following standard facts about the geometry of the product of two spheres of radii $r_{1}$ and $r_{2}$

$$
\operatorname{Isom}\left(\mathbb{S}_{r_{1}}^{p-1} \times \mathbb{S}_{r_{2}}^{q-1}\right)= \begin{cases}\mathrm{O}(p) \times \mathrm{O}(q) & \text { if } p \neq q \text { or } r_{1} \neq r_{2} \\ \mathrm{O}(p) \times \mathrm{O}(p) \rtimes_{\rho}\langle E\rangle & \text { if } p=q \text { and } r_{1}=r_{2},\end{cases}
$$

(the semidirect product structure in the latter case is discussed in more detail in (5.5)) and that for $p \neq q, \mathbb{S}_{r_{1}}^{p-1} \times \mathbb{S}_{r_{2}}^{q-1}$ and $\mathbb{S}_{r_{1}^{\prime}}^{p-1} \times \mathbb{S}_{r_{2}^{\prime}}^{q-1}$ are isometric if and only if $r_{1}=r_{1}^{\prime}$ and $r_{2}=r_{2}^{\prime}$ and for $p=q$ are isometric if and only if the sets $\left\{r_{1}, r_{2}\right\}$ and $\left\{r_{1}^{\prime}, r_{2}^{\prime}\right\}$ are the same.

Let $\mathrm{I}$ be any element in $\operatorname{Isom}\left(\mathrm{Cyl}^{p, q}, g_{\tau}\right)$. Choose any meridian $M=$ $\left\{t_{k}\right\} \times \operatorname{Mer}^{p, q}$ so that $k \in \mathbb{Z}$ satisfies $y_{\tau}\left(t_{k}\right)=y_{\text {min }}$, i.e., so that $y_{\tau}$ is minimal on $M$. We established above that I maps any meridian of $\mathrm{Cyl}^{p, q}$ to another (isometric) meridian. In particular, when $p=1$ or when $p>1$ and $p \neq q$ this implies (using the standard facts about when products of two spheres are isometric) that I maps $M$ to another meridian where $y_{\tau}$ is minimal. If $p>1$ and $p=q$ then I maps $M$ to another meridian where $y_{\tau}$ is either minimal or maximal (recall (4.11)). In any case of the three cases i-iii, it follows that by composing with a suitable isometry $\mathbf{D} \in \mathbf{D}$ we can arrange that $\mathrm{D} \circ \mathrm{I}$ fixes $M$ as a set. Hence $\mathrm{D} \circ \mathrm{I}$ restricted to $M$ yields an isometry of $M$. Since $M$ is a meridian with $y_{\tau}$ minimal by (5.13) we have $\operatorname{Isom}(M)=\mathrm{O}$ with $\mathrm{O}$ as in 5.6.i-iii. Hence there exists $\mathrm{O} \in \mathrm{O}$ such that $\mathrm{O} \circ \mathrm{D} \circ \mathrm{I}$ fixes $M$ pointwise. Therefore $\mathrm{O} \circ \mathrm{D} \circ \mathrm{I}$ sends any generator $\gamma_{\sigma}$ to itself and hence we have

$$
\mathrm{O} \circ \mathrm{D} \circ \mathbf{I}=\left\{\begin{array}{l}
\mathrm{Id} \\
\underline{\mathrm{T}}_{t_{k}}
\end{array}\right.
$$

according to whether $\mathrm{O} \circ \mathrm{D} \circ \mathrm{I}$ fixes or reflects all generators. In either case it follows that $\mathbf{I} \in \mathbf{D} \cdot \mathrm{O}$ for any $\mathbf{I} \in \operatorname{Isom}\left(\mathrm{Cyl}^{p, q}, g_{\tau}\right)$ as claimed. 
The geometry of $\mathrm{SO}(p) \times \mathrm{SO}(q)$-invariant special Lagrangian cones 211

Finally we must show that $\operatorname{Isom}\left(\mathrm{Cy}^{p}{ }^{p, q}, g_{\tau}\right)$ is isomorphic to the claimed groups. First we note that all three discrete groups $\mathbf{D}$ defined in Proposition 5.6.i-iii are isomorphic to the infinite dihedral group $\mathbf{D}_{\infty}$. This is essentially already proved in 4.13 , although when $p=q$ we are considering the subgroup of $\operatorname{Isom}\left(\mathrm{Cyl}^{p, p}\right)$ generated by $\underline{\mathrm{T}} \circ \mathrm{E}$ and ${\underline{\mathrm{p}_{\tau} / 2}}_{2}$, rather than the subgroup of $\operatorname{Isom}(\mathbb{R})$ generated by $\underline{T}$ and $\underline{T}_{\mathbf{p}_{\tau} / 2}$. Nevertheless, the same argument applies.

By 5.6.i $\operatorname{Isom}\left(\mathrm{Cyl}^{1, n-1}, g_{\tau}\right)=\mathbf{D} \cdot \mathrm{O}(n-1)$ where $\mathbf{D}=\left\langle\underline{\mathbf{T}}, \mathbf{T}_{2 \mathrm{p}_{\tau}}\right\rangle$. Since $\mathbf{D}$ acts only on the $\mathbb{R}$ factor of $\mathrm{Cyl}^{1, n-1}$ and $\mathrm{O}(n-1)$ acts only on the $\mathbb{S}^{n-2}$ factor $\mathbf{D}$ centralizes $\mathrm{O}(n-1)$ and also that $\mathbf{D} \cap \mathrm{O}(n-1)=(\mathrm{Id})$. Hence $\mathbf{D} \cdot \mathrm{O}(n-1) \cong \mathbf{D} \times \mathrm{O}(n-1)$. By 5.6.ii $\operatorname{Isom}\left(\mathrm{Cyl}^{p, q}, g_{\tau}\right)=\mathbf{D} \cdot \mathrm{O}(p) \times \mathrm{O}(q)$ where $\mathbf{D}=\left\langle\underline{\mathbf{T}}_{\mathbf{p}_{\tau}^{+}}, \underline{\mathbf{T}}_{-\mathbf{p}_{\tau}^{-}}\right\rangle$. By the same argument as above $\mathbf{D} \cdot \mathrm{O}(p) \times \mathrm{O}(q) \cong$ $\mathbf{D} \times \mathrm{O}(p) \times \mathrm{O}(q)$ By 5.6.iii $\operatorname{Isom}\left(\mathrm{Cyl}^{p, p}, g_{\tau}\right)=\mathbf{D} \cdot \mathrm{O}(p) \times \mathrm{O}(p)$ where $\mathbf{D}=$ $\left\langle\underline{\mathbf{T}} \circ \mathbf{E}, \underline{\mathbf{T}}_{\mathbf{p}_{\tau} / 2}\right\rangle$. D does not centralize $\mathrm{O}(p) \times \mathrm{O}(p)$, since $\mathbf{D}$ no longer acts only on the $\mathbb{R}$ factor of $\mathrm{Cyl}^{p, p}$. However, conjugation by any element of $\mathbf{D}$ does preserve the subgroup $\mathrm{O}(p) \times \mathrm{O}(p) \subset \operatorname{Diff}\left(\mathrm{Cyl}^{p, p}\right)$. More precisely, if $\gamma \in\left\langle\underline{\mathrm{T}} \circ \mathbf{E}, \underline{\mathbf{T}}_{\mathbf{p}_{\tau} / 2}\right\rangle$ then

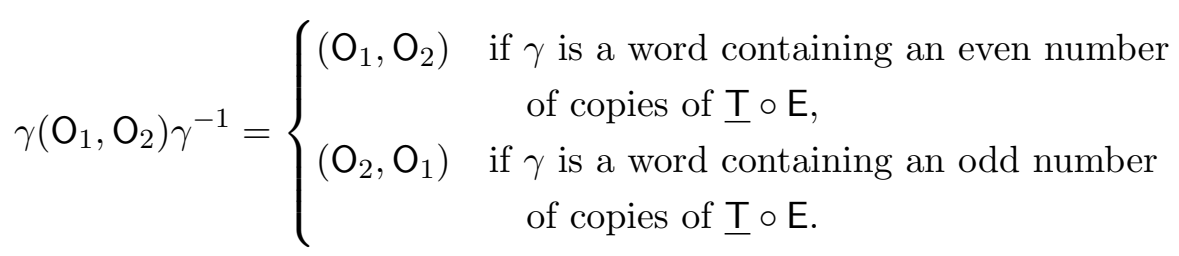

It follows that the set $\mathbf{D} \cdot \mathrm{O}(p) \times \mathrm{O}(p)$ coincides with the set $\mathrm{O}(p) \times \mathrm{O}(p) \cdot \mathbf{D}$ and hence that $\mathbf{D} \cdot \mathrm{O}(p) \times \mathrm{O}(p)$ is a group, containing the subgroup $\mathrm{O}(p) \times$ $\mathrm{O}(p)$ as a normal subgroup of this group. Since clearly $\mathrm{O}(p) \times \mathrm{O}(p) \cap \mathbf{D}=$ (Id), we have the semidirect product structure claimed.

Remark 5.14. When $|\tau|=\tau_{\max }, y_{\tau} \equiv \frac{q}{n}$ and therefore $y_{\tau}$ is invariant under the whole of $\operatorname{Isom}(\mathbb{R})$. In particular, all meridians $\{t\} \times \mathrm{Mer}^{p, q}$ of $\mathrm{Cyl}{ }^{p, q}$ are isometric. When $p \neq q$ the isometry group of each meridian is the group $\mathrm{O}$ (defined in 5.6). If $p=q$ then each meridian is a product of two $p-1$ spheres of the same radius and hence the isometry group of each meridian is the extension of $\mathrm{O}(p) \times \mathrm{O}(p)$ given in case two of (5.13). For $p \neq q$ the isometry group of $g_{\tau}$ for $|\tau|=\tau_{\max }$ is $\operatorname{Isom}(\mathbb{R}) \cdot \mathrm{O}$, whereas for $p=q$ it is $\operatorname{Isom}(\mathbb{R}) \cdot \operatorname{Isom}\left(\mathbb{S}_{r}^{p-1} \times \mathbb{S}_{r}^{p-1}\right)$. In all cases the action of the isometry group is transitive on $\mathrm{Cyl}^{p, q}$ thus making it into a Riemannian homogeneous space.

When $\tau=0$ we have from 3.30.iv that $g_{0}$ is isometric to the restriction of the round metric to $\mathbb{S}^{p+q-1} \backslash\left(\mathbb{S}^{p-1}, 0\right) \cup\left(0, \mathbb{S}^{q-1}\right)$ if $p>1$ or to $\mathbb{S}^{n-1} \backslash( \pm 1,0)$ if $p=1$. Hence for $p=1$ we have $\operatorname{Isom}\left(\mathrm{Cyl}^{1, n-1}, g_{0}\right) \cong \mathrm{O}(1) \times \mathrm{O}(n-1)$ the subgroup of $\mathrm{O}(n)$ leaving invariant the line through $e_{1}$ (the $\mathrm{O}(1)$ factor 
being generated by $\left.\underline{\mathrm{T}} \in \operatorname{Diff}\left(\mathrm{Cyl}^{1, n-1}\right)\right)$. Similarly, for $p>1$ and $p \neq q$ we have $\operatorname{Isom}\left(\mathrm{Cyl}^{p, q}, g_{0}\right)=\mathrm{O}(p) \times \mathrm{O}(q)$, the subgroup of $\mathrm{O}(n)$ leaving invariant the subset $\left(\mathbb{S}^{p-1}, 0\right) \cup\left(0, \mathbb{S}^{q-1}\right) \subset \mathbb{S}^{p+q-1}$. Finally, for $p>1$ and $p=q$ we have $\operatorname{Isom}\left(\mathrm{Cyl}^{p, p}, g_{0}\right)=\langle\underline{\mathrm{T}} \circ \mathrm{E}\rangle \cdot \mathrm{O}(p) \times \mathrm{O}(p)$, which is isomorphic to the subgroup of $\mathrm{O}(n)$ leaving invariant the subset $\left(\mathbb{S}^{p-1}, 0\right) \cup\left(0, \mathbb{S}^{p-1}\right) \subset \mathbb{S}^{2 p-1}$.

Discrete symmetries of $\boldsymbol{X}_{\boldsymbol{\tau}}$. In this section we exhibit all the discrete symmetries enjoyed by $X_{\tau}$. In particular, we establish that $\operatorname{Sym}\left(X_{\tau}\right)=$ Isom $\left(\mathrm{Cyl}^{p, q}, g_{\tau}\right)$. We begin by describing the discrete symmetries of $X_{\tau}$ for the case $p=1$.

Proposition 5.15 (Discrete symmetries of $\boldsymbol{X}_{\boldsymbol{\tau}}$ for $\boldsymbol{p}=\mathbf{1}$ ). For $p=1$ and $0<|\tau|<\tau_{\max }, X_{\tau}$ admits the following symmetries

$$
\begin{aligned}
\widetilde{\mathrm{O}} \circ X_{\tau} & =X_{\tau} \circ \mathrm{O}, \quad \text { for all } \mathrm{O} \in O(n-1), \\
\widetilde{\mathrm{T}}_{2 \widehat{\mathrm{p}}_{\tau}} \circ X_{\tau} & =X_{\tau} \circ \mathrm{T}_{2 \mathrm{p}_{\tau}}, \\
\widetilde{\mathbf{T}} \circ X_{\tau} & =X_{\tau} \circ \underline{\mathrm{T}}, \\
\widetilde{\mathbf{T}}_{\widehat{\mathrm{p}}_{\tau}} \circ X_{\tau} & =X_{\tau} \circ \underline{\mathrm{T}}_{\mathrm{p}_{\tau}},
\end{aligned}
$$

where $\widetilde{\mathbf{T}}_{x} \in S U(n)$ is defined in (3.7), $\widetilde{\mathbf{T}} \in O(2 n)$ and $\widetilde{\mathbf{T}}_{x} \in O(2 n)$ are defined by

$$
\begin{gathered}
\underline{\widetilde{\mathbf{T}}}\left(z_{1}, \ldots, z_{n}\right)=\left(-\bar{z}_{1}, \bar{z}_{2}, \ldots, \bar{z}_{n}\right), \\
\underline{\widetilde{\mathbf{T}}}_{x}=\widetilde{\mathrm{T}}_{2 x} \circ \underline{\widetilde{\mathrm{T}}} .
\end{gathered}
$$

Proof. The $\mathrm{O}(n-1)$-equivariance expressed by (5.16a) follows immediately from the definition of $X_{\tau}$ (and extends the $\mathrm{SO}(n-1)$-invariance used to construct $X_{\tau}$ in the first place). The symmetries $(5.16 \mathrm{~b})-(5.16 \mathrm{~d})$ of $X_{\tau}$ are equivalent to the three symmetries of $\mathbf{w}_{\tau}$ established in (4.27).

\section{Remark 5.19.}

(i) Symmetries when $\tau=0$ : from 3.30.iv $X_{0}: \mathrm{Cyl}^{1, n-1} \rightarrow \mathbb{S}^{2 n-1}$ is an embedding whose image is the totally real equatorial sphere $\mathbb{S}^{n-1} \subset$ $\mathbb{R}^{n} \subset \mathbb{C}^{n}$ minus the two antipodal points $\pm e_{1}$. Clearly the subgroup $\mathrm{O}(n) \subset \mathrm{O}(2 n)$ leaves this equatorial $n-1$ sphere invariant. $\mathrm{O}(n-1) \subset \mathrm{O}(n)$ is the subgroup of $\mathrm{O}(n)$ fixing the line spanned by $e_{1}$. There is no analogue of the symmetries in (5.16b) and (5.16d) in 
The geometry of $\mathrm{SO}(p) \times \mathrm{SO}(q)$-invariant special Lagrangian cones 213

this case since the period $2 \mathrm{p}_{\tau} \rightarrow \infty$ as $\tau \rightarrow 0$ (see Section 7 ). However, the isometry $\widetilde{\mathbf{I}} \in \mathrm{O}(2 n)$ leaves $\mathbb{S}^{n-1}$ invariant and sends $e_{1}$ to $-e_{1}$ (cf. Remark 5.14). Hence the symmetry (5.16c) still holds in the case $\tau=0$. This symmetry is equivalent to the fact that $y_{0}$ is even in the case $p=1$ (recall 3.18.iv).

(ii) Symmetries when $|\tau|=\tau_{\max }$ : In this case $y_{\tau}$ is constant and hence $X_{\tau}$ has the additional continuous symmetries

$$
\widetilde{\mathrm{T}}_{x} \circ X_{\tau}=X_{\tau} \circ \mathrm{T}_{x}, \quad \text { for all } x \in \mathbb{R} .
$$

The discrete symmetry (5.16c) still holds in this case and so the analogue of (5.16d) holds for all $x \in \mathbb{R}$.

Using the Definitions (3.7), (5.17) and (5.18) it is easy to check the following

Proposition 5.20 (Properties of discrete symmetries of target for $p=1)$.

(i) $\widetilde{\underline{I}} \circ \widetilde{\mathrm{T}}_{x} \circ \widetilde{\mathrm{I}}=\widetilde{\mathrm{T}}_{-x}$.

(ii) The $O(2)$ subgroup generated by $\widetilde{\mathbf{I}}$ and $\left\{\widetilde{\mathbf{T}}_{x}\right\}$ centralizes $O(n-1) \subset$ $O(n) \subset O(2 n)$.

(iii) $\widetilde{\mathrm{T}}_{x}$ commutes with $J$, while $\widetilde{\mathrm{I}}$ and $\widetilde{\mathrm{I}}_{x}$ anticommute with $J$.

(iv) $\widetilde{\mathrm{T}}_{x}$ preserves both $\Omega$ and $\omega$.

(v) $\widetilde{\mathrm{I}}^{*} \Omega=-\bar{\Omega}, \quad \widetilde{\mathbf{I}}^{*} \omega=-\omega$.

Remark 5.21. We see from 5.20.v that $\widetilde{\mathbf{I}}$ is an anti-SL anti-holomorphic isometry, while for any $x \in \mathbb{R}, \widetilde{\mathrm{T}}_{x} \in \mathrm{SU}(n) \subset \operatorname{Isom}_{\mathrm{SL}} \subset \operatorname{Isom}_{ \pm \mathrm{SL}}$. Since $\underline{\mathrm{T}}$ is reflection in the origin in $\mathbb{R}$, we have the commutation relation $\mathrm{T} \circ \mathrm{T}_{x} \circ \overline{\mathrm{I}}=$ $\mathrm{T}_{-x}$. Part (i) above says that the same relations also hold for $\widetilde{\mathrm{T}}$ and $\widetilde{\mathrm{T}}_{x}$ and that $\widetilde{T}_{x}$ and $\widetilde{I}$ generate a subgroup of $\mathrm{O}(2 n)$ isomorphic to $\mathrm{O}(2) \cong \mathbb{S}^{1} \rtimes \mathbb{Z}_{2}$, where $\widetilde{\underline{I}}$ generates the $\mathbb{Z}_{2}$ factor and acts by inversion (thinking of the group generated by $\widetilde{\mathrm{T}}_{x}$ as an abelian group) on the $\mathbb{S}^{1}$ factor. Also, since they act on different factors of $\mathbb{R} \times \mathbb{S}^{n-2}$, every element in $\operatorname{Isom}(\mathbb{R}) \subset \operatorname{Isom}\left(\mathrm{Cyl} 1^{1, n-1}\right.$ ) commutes with every element in $\mathrm{O}(n-1) \subset \operatorname{Isom}\left(\mathrm{Cyl}^{1, n-1}\right)$. Part (ii) above is the analogue of this result for the group $\mathrm{O}(2)$ generated by $\widetilde{\mathbf{I}}$ and $\widetilde{\mathbf{T}}_{x}$.

We now describe the discrete symmetries of $X_{\tau}$ in the case $p>1$ and $p \neq q$, cf. Prop. 5.15 . 
Proposition 5.22 (Discrete symmetries of $X_{\tau}$ for $p>1$; cf. Prop. 5.15). For $p>1$ and $0<|\tau|<\tau_{\max }, X_{\tau}$ admits the following symmetries

$$
\begin{aligned}
\widetilde{\mathrm{O}} \circ X_{\tau} & =X_{\tau} \circ \mathrm{O}, \quad \text { for all } \mathrm{O} \in O(p) \times O(q), \\
\widetilde{\mathrm{T}}_{2 \widehat{\mathrm{p}}_{\tau}} \circ X_{\tau} & =X_{\tau} \circ \mathrm{T}_{2 \mathrm{p}_{\tau}}, \\
\widetilde{\mathbf{I}}_{+} \circ X_{\tau} & =X_{\tau} \circ \underline{\mathrm{I}}_{\mathrm{p}_{\tau}^{+}}, \\
\widetilde{\mathbf{I}}_{-} \circ X_{\tau} & =X_{\tau} \circ \underline{\mathrm{I}}_{-\mathrm{p}_{\tau}^{-}},
\end{aligned}
$$

where $\widetilde{\mathrm{T}}_{x} \in S U(n)$ is defined by (3.7) and $\widetilde{\mathrm{I}}_{+}, \widetilde{\mathrm{I}}_{-} \in O(2 n)$ (which depend on $\tau$ ) are defined by

$$
\begin{aligned}
& \widetilde{\mathbf{I}}_{+}(z, w)=\left(\mathrm{e}^{\mathrm{i} \alpha_{\tau} / p} \mathrm{e}^{\mathrm{i} \psi_{1}\left(2 \mathrm{p}_{\tau}^{+}\right)} \bar{z}, \mathrm{e}^{\mathrm{i} \alpha_{\tau} / q} \mathrm{e}^{\mathrm{i} \psi_{2}\left(2 \mathrm{p}_{\tau}^{+}\right)} \bar{w}\right), \\
& \widetilde{\mathbf{I}}_{-}(z, w)=\left(\mathrm{e}^{\mathrm{i} \alpha_{\tau} / p} \mathrm{e}^{\mathrm{i} \psi_{1}\left(-2 \mathbf{p}_{\tau}^{-}\right)} \bar{z}, \mathrm{e}^{\mathrm{i} \alpha_{\tau} / q} \mathrm{e}^{\mathrm{i} \psi_{2}\left(-2 \mathbf{p}_{\tau}^{-}\right)} \bar{w}\right),
\end{aligned}
$$

where $z \in \mathbb{C}^{p}$ and $w \in \mathbb{C}^{q}$.

Proof. (5.23a) follows immediately from the definition of $X_{\tau}$ (and extends the $\mathrm{SO}(p) \times \mathrm{SO}(q)$-invariance used to construct $X_{\tau}$ in the first place). The symmetries $(5.23 \mathrm{~b}),(5.23 \mathrm{c})$ and $(5.23 \mathrm{~d})$ of $X_{\tau}$ are equivalent to the symmetries of $\mathbf{w}_{\tau}$ established in (4.30).

\section{Remark 5.25.}

(i) Symmetries when $\tau=0$ : by 3.30.iv $X_{0}: \mathrm{Cy}{ }^{p, q} \rightarrow \mathbb{S}^{2 n-1}$ is an embedding whose image is the standard equatorial sphere $\mathbb{S}^{n-1} \subset \mathbb{R}^{n} \subset \mathbb{C}^{n}$ minus the two equatorial subspheres $\mathbb{S}^{n-1} \cap\left(\mathbb{R}^{p}, 0\right)$ and $\mathbb{S}^{n-1} \cap\left(0, \mathbb{R}^{q}\right)$. Clearly the subgroup $\mathrm{O}(n) \subset \mathrm{O}(2 n)$ leaves this equatorial $n-1$ sphere invariant. $\mathrm{O}(p) \times \mathrm{O}(q) \subset \mathrm{O}(n)$ is the subgroup of $\mathrm{O}(n)$ fixing this distinguished pair of orthogonal equatorial subspheres. Therefore $X_{0}$ is still $\mathrm{O}(p) \times \mathrm{O}(q)$-equivariant as in (5.23a). However, there is no analogue of any of the other symmetries in 5.23 in this case. This is consistent with (recall 5.14) $\operatorname{Isom}\left(\mathrm{Cyl}^{p, q}, g_{0}\right)=\mathrm{O}(p) \times \mathrm{O}(q)$ when $p>1$ and $p \neq q$.

(ii) Symmetries when $|\tau|=\tau_{\max }$ : As in the $p=1$ case discussed in Remark $5.19 y_{\tau}$ is constant and hence $X_{\tau}$ has the additional continuous symmetries

$$
\widetilde{\mathrm{T}}_{x} \circ X_{\tau}=X_{\tau} \circ \mathrm{T}_{x}, \quad \text { for all } x \in \mathbb{R},
$$


The geometry of $\mathrm{SO}(p) \times \mathrm{SO}(q)$-invariant special Lagrangian cones 215

and $X_{\tau}$ is therefore homogeneous rather than cohomogeneity one as for other values of $\tau$.

Using Definitions (3.7), (5.24a) and (5.24b) one can check the following:

Proposition 5.26 (Properties of target discrete symmetries for $p>1, p \neq q$, cf. 5.20).

(i) $\underline{\widetilde{I}}_{+} \circ \widetilde{\mathrm{T}}_{x} \circ \widetilde{\mathrm{I}}_{+}=\widetilde{\mathrm{I}}_{-} \circ \widetilde{\mathrm{T}}_{x} \circ \widetilde{\mathrm{I}}_{-}=\widetilde{\mathrm{T}}_{-x}$.

(ii) $\underline{\widetilde{I}}_{-} \circ \underline{\underline{I}}_{+}=\widetilde{T}_{-2 \widehat{p}_{\tau}}, \quad \underline{\mathbf{I}}_{+} \circ \underline{\mathbf{I}}_{-}=\widetilde{\mathrm{T}}_{2 \widehat{\mathrm{p}}_{\tau}}$.

(iii) the dihedral subgroup $\widetilde{\mathbf{D}}:=\left\langle\widetilde{\mathbf{T}}_{+}, \widetilde{\mathbf{I}}_{-}\right\rangle$centralizes $O(p) \times O(q) \subset O(n) \subset$ $O(2 n)$.

(iv) $\widetilde{\mathrm{T}}_{x}$ commutes with $J$, while $\widetilde{\mathrm{I}}_{+}$and $\widetilde{\mathrm{I}}_{-}$anticommute with $J$.

(v) $\widetilde{\mathrm{T}}_{x}$ preserves both $\Omega$ and $\omega$.

(vi) $\underline{\underline{\mathbf{I}}}_{+}^{*} \Omega=\widetilde{\mathbf{I}}_{-}^{*} \Omega=-\bar{\Omega}, \quad \widetilde{\mathbf{I}}_{+}^{*} \omega=\widetilde{\mathbf{I}}_{-}^{*} \omega=-\omega$.

To prove the first two equalities of 5.26.vi one also needs to use (4.21a), (4.21b) and (4.36). 5.26.vi implies that both $\widetilde{\mathbf{I}}_{+}$and $\widetilde{\mathbf{I}}_{-}$are anti-SL, antiholomorphic isometries in $\mathrm{Isom}_{ \pm \mathrm{SL}}$.

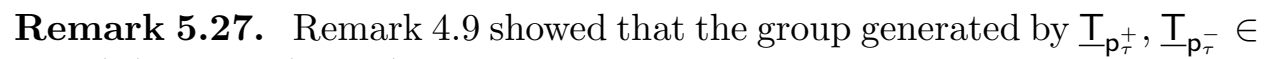
$\operatorname{Isom}(\mathbb{R}) \subset \operatorname{Isom}\left(\mathrm{Cyl}^{p, q}\right)$ is isomorphic to the infinite dihedral group $\mathbf{D}_{\infty}$. Part (ii) above gives the analogous result for the subgroup $\widetilde{\mathbf{D}}$ of $\mathrm{O}(2 n)$ generated by $\widetilde{\mathbf{I}}_{+}$and $\widetilde{\mathbf{I}}_{-}$. See Lemma 5.38 for the precise structure of $\widetilde{\mathbf{D}}$. Part (iii) is the analogue of the fact that $\mathbf{D}$ and $\mathrm{O}(p) \times \mathrm{O}(q) \subset \operatorname{Isom}\left(\mathrm{Cyl}^{p, q}\right)$ centralize each other.

Finally, we describe the discrete symmetries of $X_{\tau}$ in the case $p>1$ and $p=q$.

Proposition 5.28 (Discrete symmetries of $X_{\tau}$ for $p=q$; cf. Props. 5.15 and 5.22). For $p>1, p=q$ and $0<|\tau|<\tau_{\max }, X_{\tau}$ admits the following symmetries

$$
\begin{aligned}
\widetilde{\mathrm{O}}_{0} \circ X_{\tau} & =X_{\tau} \circ \mathrm{O}, \quad \text { for all } \mathrm{O} \in O(p) \times O(p), \\
\widetilde{\mathrm{T}}_{2 \widehat{\mathrm{p}}_{\tau}} \circ X_{\tau} & =X_{\tau} \circ \mathrm{T}_{2 \mathrm{p}_{\tau}}, \\
\widetilde{\mathrm{I}}_{+} \circ X_{\tau} & =X_{\tau} \circ \underline{\mathrm{I}}_{\mathrm{p}_{\tau} / 2},
\end{aligned}
$$




$$
\begin{aligned}
\underline{\mathrm{I}}_{-} \circ X_{\tau} & =X_{\tau} \circ \underline{\mathrm{I}}-\mathrm{p}_{\tau} / 2, \\
\underline{\mathrm{I}} \circ X_{\tau} & =X_{\tau} \circ \underline{\mathrm{I}} \circ \mathrm{E}, \\
\widetilde{\mathrm{T}}_{\hat{\mathrm{p}}_{\tau}} \circ \underline{\widetilde{\mathrm{S}}} \circ X_{\tau} & =X_{\tau} \circ \mathrm{T}_{\mathrm{p}_{\tau}} \circ \mathrm{E},
\end{aligned}
$$

where $\widetilde{\mathrm{T}}_{x}$, $\underline{\mathbf{T}}_{+}$and $\underline{\mathrm{T}}_{-}$are defined as in 5.22, $\underline{\mathbf{T}} \in O(2 p) \subset U(2 p)$ and $\underline{\widetilde{\mathrm{S}}} \in$ $O(4 p)$ are defined by

$$
\begin{gathered}
\widetilde{\mathbf{T}}(z, w)=(w, z) ; \\
\underline{\widetilde{S}}(z, w)=\mathrm{e}^{-\mathrm{i} \pi / 2 p}(\bar{w}, \bar{z}),
\end{gathered}
$$

where $w, z \in \mathbb{C}^{p}$. Furthermore, the reflections $\underline{\mathbf{I}}_{+}$and $\underline{\mathbf{I}}_{-}$can also be expressed as

$$
\begin{aligned}
& \widetilde{T}_{+}=\widetilde{T}_{\hat{\mathrm{p}}_{\tau}} \circ \underline{\widetilde{S}} \circ \underline{\widetilde{T}}, \\
& \underline{\widetilde{T}}-=\widetilde{T}_{-\hat{p}_{\tau}} \circ \underline{\widetilde{S}} \circ \underline{\widetilde{T}} .
\end{aligned}
$$

Proof. The $\mathrm{O}(p) \times \mathrm{O}(p)$-equivariance expressed by (5.29a) follows as a special case of (5.23a). Similarly, since $\mathrm{p}_{\tau}^{+}=\mathrm{p}_{\tau}^{-}=\frac{1}{2} \mathrm{p}_{\tau},(5.29 \mathrm{~b})-(5.29 \mathrm{~d})$ are each special cases of $(5.23 \mathrm{~b}),(5.23 \mathrm{c})$ and $(5.23 \mathrm{~d})$ respectively. $(5.29 \mathrm{e})$ is equivalent to the symmetry of $\mathbf{w}_{\tau}$ with respect to $\mathrm{T}$ given in $(4.31)$. (5.29f) follows from the symmetries $(5.29 \mathrm{c})$ and $(5.29 \mathrm{e})$, using $(5.32 \mathrm{a})$.

Remark 5.33. (5.29e) and (5.29f) express the two additional symmetries that $X_{\tau}$ possesses when $p=q$ and both utilize the additional exchange isometry $\mathrm{E} \in \operatorname{Isom}\left(\mathrm{Cyl}^{p, p}\right)$. (5.29e) expresses an additional reflectional symmetry of $X_{\tau}$ about the $\mathrm{SO}(p) \times \mathrm{SO}(p)$-orbit for which the radii of both $p-1$ spheres are equal.

Using (5.30)-(5.32b), one can check the following:

Proposition 5.34 (Properties of target discrete symmetries for $\boldsymbol{p}=\boldsymbol{q}$, cf. $\mathbf{5 . 2 0}, \mathbf{5 . 2 6})$. $\underline{\mathrm{T}}_{+}, \underline{\mathrm{T}}_{-}, \widetilde{\mathrm{T}}_{x}$ have all the properties detailed in Proposition 5.26. Additionally the new isometries $\widetilde{\widetilde{S}}$ and $\widetilde{\mathrm{I}}$ satisfy

(i) $\underline{\underline{\mathrm{T}}} \circ \widetilde{\mathrm{T}}_{x} \circ \underline{\underline{\mathrm{T}}}=\widetilde{\mathrm{T}}_{-x}$ and $\underline{\underline{\mathrm{T}}} \circ \underline{\mathrm{T}}_{+} \circ \underline{\tilde{\mathrm{T}}}=\underline{\mathrm{T}}_{-}$.

(ii) $\underline{\widetilde{S}}$ commutes with $\underline{\mathbf{T}}_{+}, \underline{\mathbf{I}}_{-}, \widetilde{\mathrm{T}}_{\text {and }}$ with $\widetilde{\mathrm{T}}_{x}$.

(iii) $\widetilde{\underline{I}}$ commutes with $J$ while $\underline{\widetilde{S}}$ anticommutes with $J$. 
The geometry of $\mathrm{SO}(p) \times \mathrm{SO}(q)$-invariant special Lagrangian cones 217

(iv) $\underline{\widetilde{\mathrm{S}}}^{*} \Omega=(-1)^{p-1} \bar{\Omega}, \quad \underline{\widetilde{\mathrm{S}}}^{*} \omega=-\omega, \quad \widetilde{\mathbf{I}}^{*} \Omega=(-1)^{p} \Omega, \quad \widetilde{\underline{\mathrm{I}}}^{*} \omega=\omega$.

(v) Conjugation by $\widetilde{\mathrm{I}}$ acts on $O(p) \times O(p) \subset O(2 p)$ by $\left(\widetilde{\mathrm{O}}_{1}, \widetilde{\mathrm{O}}_{2}\right) \mapsto\left(\widetilde{\mathrm{O}}_{2}, \widetilde{\mathrm{O}}_{1}\right)$.

5.34.iv implies that $\widetilde{\mathrm{I}} \in \mathrm{SU}(2 p)^{ \pm}=\operatorname{Isom}_{ \pm \mathrm{SL}}^{J}$ and that $\widetilde{\mathrm{I}} \in \mathrm{SU}(2 p)$ if and only if $p$ is even.

The structure of $\widetilde{\operatorname{Sym}}\left(X_{\tau}\right)$. Now, we determine the structure of $\widetilde{\operatorname{Sym}}\left(X_{\tau}\right) \subset \mathrm{O}(2 n)($ recall $(2.9))$ as an abstract group.

Definition 5.35. Define a subgroup $\widetilde{\mathbf{D}} \subset \widetilde{\operatorname{Sym}}\left(X_{\tau}\right)$ by $\widetilde{\mathbf{D}}:=\widetilde{\rho}(\mathbf{D})$ where $\widetilde{\rho}: \operatorname{Sym}\left(X_{\tau}\right) \rightarrow \widetilde{\operatorname{Sym}}\left(X_{\tau}\right)$ is the epimorphism described in 2.10. More concretely, using $5.15,5.22$ and 5.28 we have

$$
\widetilde{\mathbf{D}}= \begin{cases}\left\langle\widetilde{\mathbf{I}}, \widetilde{\mathbf{T}}_{2 \widehat{\mathbf{p}}_{\tau}}\right\rangle & \text { if } p=1 ; \\ \left\langle\widetilde{\mathbf{I}}_{+}, \widetilde{\mathbf{I}}_{-}\right\rangle & \text {if } p>1 \text { and } p \neq q ; \\ \left\langle\widetilde{\mathbf{I}}, \widetilde{\mathbf{I}}_{+}\right\rangle & \text {if } p>1 \text { and } p=q ;\end{cases}
$$

respectively, where $\widetilde{\mathrm{T}}_{x} \in \mathrm{SU}(n)$ is defined in (3.7) and $2 \widehat{\mathrm{p}}_{\tau}$ is the angular period defined in (4.23).

Using 5.20, 5.26 and 5.34, we see that in each case the generators of $\widetilde{\mathbf{D}}$ belong to $\mathrm{Isom}_{ \pm \mathrm{SL}}$ and hence $\widetilde{\mathbf{D}} \subset \mathrm{Isom}_{ \pm \mathrm{SL}}$.

In all three cases $\widetilde{\mathrm{T}}_{2 \widehat{\mathrm{p}}_{\tau}}=\widetilde{\rho}\left(\mathrm{T}_{2 \mathrm{p}_{\tau}}\right) \in \widetilde{\mathbf{D}} \subset \widetilde{\operatorname{Sym}}\left(X_{\tau}\right)$ for $0<|\tau|<\tau_{\max }$. Therefore for any such $\tau$ we call the immersion $X_{\tau}$ : $\mathrm{Cy}^{p, q} \rightarrow \mathbb{S}^{2(p+q)-1}$ $2 \mathrm{p}_{\tau}$-periodic; the corresponding element $\widetilde{\mathrm{T}}_{2 \widehat{\mathrm{p}}_{\tau}} \in \mathrm{SU}(n)$, we call the rotational period of $X_{\tau}$. In 4.48 we defined the rotational period $\hat{\mathrm{T}}_{2 \widehat{\mathfrak{p}}_{\tau}} \in \mathrm{U}(2)$ of $\mathbf{w}_{\tau}$ and also its order $k_{0} \in \mathbb{N} \cup\{\infty\}$. Using the definition of $X_{\tau}$ in terms of the curve $\mathbf{w}_{\tau}$ the order of the rotational period $\hat{T}_{2 \widehat{\mathrm{p}}_{\tau}} \in \mathrm{U}(2)$ of $\mathbf{w}_{\tau}$ is equal to the order of the rotational period $\widetilde{\mathrm{T}}_{2 \widehat{\mathrm{p}}_{\tau}} \in \mathrm{SU}(n)$ of $X_{\tau}$. Hence, we will simply refer to $k_{0}$ as the order of the rotational period.

Let $\widetilde{O} \subset \mathrm{O}(n) \subset \mathrm{SU}(n)^{ \pm} \subset \mathrm{O}(2 n)$ denote $\mathrm{O}(n-1) \subset \mathrm{O}(n)$ if $p=1$ or $\mathrm{O}(p) \times \mathrm{O}(q) \subset \mathrm{O}(n)$ if $p>1$. Then $\widetilde{O}=\widetilde{\rho}(O) \subset \widetilde{\operatorname{Sym}}\left(X_{\tau}\right)$ where $O$ is the subgroup of $\operatorname{Sym}\left(X_{\tau}\right)$ defined in 5.6.i-iii; moreover $\widetilde{O}$ and $O$ are isomorphic. Hence we have

$$
\widetilde{\operatorname{Sym}}\left(X_{\tau}\right)=\widetilde{\rho}\left(\operatorname{Sym}\left(X_{\tau}\right)\right)=\widetilde{\rho}(\mathbf{D} \cdot O)=\widetilde{\rho}(\mathbf{D}) \cdot \widetilde{\rho}(O)=\widetilde{\mathbf{D}} \cdot \widetilde{O} \subset \operatorname{Isom}_{ \pm \mathrm{SL}} .
$$


To determine the structure of $\widetilde{\operatorname{Sym}}\left(X_{\tau}\right)$ as an abstract group it suffices to understand the structure of the subgroup $\widetilde{\mathbf{D}}$, the commutation relations between $\widetilde{\mathbf{D}}$ and $\widetilde{O}$ and the intersection $\widetilde{\mathbf{D}} \cap \widetilde{O}$.

Lemma 5.38. For $0<|\tau|<\tau_{\max }$ we have:

(i) The subgroup $\widetilde{\mathbf{D}} \subset \widetilde{\operatorname{Sym}}\left(X_{\tau}\right)$ (recall 5.35) has the structure

$$
\widetilde{\mathbf{D}} \cong \begin{cases}\mathbf{D}_{\infty} & \text { if } k_{0} \text { is infinite; } \\ \mathbf{D}_{k_{0}} & \text { if } k_{0} \text { is finite and } p=1 \text { or } p>1 \text { and } p \neq q ; \\ \mathbf{D}_{2 k_{0}} & \text { if } k_{0} \text { is finite and } p>1 \text { and } p=q .\end{cases}
$$

(ii)

$$
\begin{aligned}
\widetilde{\mathbf{D}} \cap \operatorname{Isom}_{ \pm S L}^{J} & =\widetilde{\mathbf{D}} \cap S U(n)^{ \pm} \\
& = \begin{cases}\left\langle\widetilde{\mathbf{T}}_{2 \widehat{\mathbf{p}}_{\tau}}\right\rangle & \text { if } p=1 \text { or if } p>1 \text { and } p \neq q ; \\
\left\langle\widetilde{\mathbf{T}}, \widetilde{\mathbf{T}}_{2 \widehat{\mathbf{p}}_{\tau}}\right\rangle & \text { if } p>1 \text { and } p=q .\end{cases}
\end{aligned}
$$

(iii) If $p=1$ or $p \geq 1$ and $\underset{\sim}{p} \neq q$ then $\widetilde{\mathbf{D}}$ centralizes $\widetilde{O}$. If $p>1$ and $p=q$ then for $\widetilde{\mathrm{M}} \in \widetilde{\mathbf{D}}=\left\langle\underline{\widetilde{\mathrm{T}}}, \underline{\mathbf{T}}_{+}\right\rangle$we have

$$
\widetilde{\mathrm{M}}\left(\widetilde{\mathrm{O}}_{1}, \widetilde{\mathrm{O}}_{2}\right) \widetilde{\mathrm{M}}^{-1}=\left\{\begin{array}{l}
\left(\widetilde{\mathrm{O}}_{1}, \widetilde{\mathrm{O}}_{2}\right) \quad \text { if } \widetilde{\mathrm{M}} \text { is a word containing an even number } \\
\quad \text { of copies of } \underline{\widetilde{\mathrm{T}}} ; \\
\left(\widetilde{\mathrm{O}}_{2}, \widetilde{\mathrm{O}}_{1}\right) \quad \text { if } \widetilde{\mathrm{M}} \text { is a word containing an odd number } \\
\text { of copies of } \underline{\mathrm{T}},
\end{array}\right.
$$

for $\operatorname{any}\left(\widetilde{\mathrm{O}}_{1}, \widetilde{\mathrm{O}}_{2}\right) \in \widetilde{O}$.

$$
\widetilde{\mathbf{D}} \cap \widetilde{O}= \begin{cases}(\mathrm{Id}) & \text { if } k_{0} \text { is infinite or odd or } p=1 \text { and } n \text { is even; } \\ \left\langle\widetilde{\mathrm{T}}_{k_{0} \widehat{\mathbf{p}}_{\tau}}\right\rangle \cong \mathbb{Z}_{2} \quad \text { if } k_{0} \text { is even and either } p>1 \text { or } p=1 \\ \text { and } n \text { is odd. }\end{cases}
$$

Proof. (i) Consider first the case where $p=1$ : recall the presentation $\left\langle r, f \mid r^{k}=1, f^{2}=1, f r f=f^{-1}\right\rangle$ for the finite dihedral group $\mathbf{D}_{k}$ and the presentation $\left\langle r, f\left\lfloor f^{2}=1, f r f=f^{-1}\right\rangle\right.$ for the infinite dihedral group $\mathbf{D}_{\infty}$. The structure of $\widetilde{\mathbf{D}}=\left\langle\underline{\mathbf{T}}, \widetilde{\mathbf{T}}_{2 \widehat{\mathbf{p}}_{\tau}}\right\rangle$ claimed now follows from 5.20.i, 5.35 and the definition of $k_{0}$. For the case $p>1$ and $p \neq q$ recall the presentation 
$\left\langle x, y \mid x^{2}=y^{2}=(x y)^{k}=1\right\rangle$ for the finite dihedral group $\mathbf{D}_{k}$ and the presentation $\left\langle x, y \mid x^{2}=y^{2}=1\right\rangle$ for the infinite dihedral group $\mathbf{D}_{\infty}$. Since by 5.26 .ii $\widetilde{\mathbf{I}}_{+} \circ \widetilde{\mathbf{I}}_{-}=\widetilde{\mathrm{T}}_{2 \widehat{\mathrm{p}}_{\tau}}$ we have $\widetilde{\mathbf{D}}=\left\langle\widetilde{\mathbf{I}}_{+}, \widetilde{\mathbf{I}}_{-}\right\rangle \cong \mathbf{D}_{k_{0}}$ if the rotational period has finite order $k_{0}$ and $\widetilde{\mathbf{D}} \cong \mathbf{D}_{\infty}$ otherwise. For the case $p>1$ and $p=q$ : using the same presentations for $\mathbf{D}_{k}$ and $\mathbf{D}_{\infty}$ as in the previous case we see that $\widetilde{\mathbf{D}}=\left\langle\widetilde{\mathbf{I}}, \widetilde{\mathbf{I}}_{+}\right\rangle$is isomorphic to a finite or infinite dihedral group depending on whether or not $\left(\widetilde{\mathbf{I}}_{+} \circ \widetilde{\mathbf{I}}\right)^{k}=\mathrm{Id}$ for some $k \in \mathbb{Z}$. From 5.34.i and 5.26.ii we have

$$
\left(\underline{\widetilde{I}}_{+} \circ \underline{\widetilde{I}}\right)^{2}=\widetilde{\mathbf{I}}_{+} \circ \underline{\mathbf{I}}_{-}=\widetilde{\mathrm{T}}_{2 \widehat{\mathrm{p}}_{\tau}} .
$$

Hence, if $k_{0}$ is finite then by the definition of $k_{0}$ we have $(\widetilde{\mathbf{T}}+\circ \widetilde{\mathbf{I}})^{2 k_{0}}=\mathrm{Id}$ and $\left(\widetilde{\mathbf{I}}_{+} \circ \widetilde{\mathbf{I}}\right)^{2 k} \neq \mathrm{Id}$ for $k<k_{0}$. By 5.26.iv and 5.34.iii $\widetilde{\mathrm{I}}$ and $\underline{\widetilde{I}}_{+}$are holomorphic and antiholomorphic respectively. Hence $\left(\widetilde{\mathbf{I}}_{+} \circ \widetilde{\mathbf{I}}\right)^{k}$ is antiholomorphic for any odd integer $k$ and so cannot be the identity. Therefore $\left(\widetilde{I}_{+} \circ \underline{\mathbf{I}}\right)$ has order exactly $2 k_{0}$ as claimed. If $k_{0}$ is infinite then $\widetilde{I}_{+} \circ \widetilde{I}$ cannot have finite order (the order cannot be odd by the antiholomorphic argument above and by 5.40 an even order would imply $k_{0}$ is finite) and hence $\widetilde{\mathbf{D}} \cong \mathbf{D}_{\infty}$ as claimed.

(ii) The case $p=1$ : Any element in $\widetilde{\mathbf{D}}=\left\langle\widetilde{\mathrm{T}}_{2 \widehat{\mathrm{p}}_{\tau}}, \widetilde{\mathbf{T}}\right\rangle$ is of the form $\widetilde{\mathbf{I}}_{k \widehat{\mathrm{p}}_{\tau}}$ (recall 5.18) or $\widetilde{\mathrm{T}}_{2 k \widehat{\mathrm{p}}_{\tau}}$ for some $k \in \mathbb{Z}$. By 5.20.iii $\widetilde{\mathbf{I}}_{k \widehat{\mathrm{p}}_{\tau}}$ acts antiholomorphically while $\widetilde{\mathrm{T}}_{2 k \widehat{\mathrm{p}}_{\tau}}$ acts holomorphically. Hence $\widetilde{\mathbf{D}} \cap \operatorname{Isom}_{ \pm \mathrm{SL}}^{J}=\left\langle\widetilde{\mathrm{T}}_{2 \widehat{\mathrm{p}}_{\tau}}\right\rangle \subset$ $\mathrm{SU}(n)$ as claimed. The case $p>1$ and $p \neq q$ : Any nontrivial element in $\widetilde{\mathbf{D}}$ can be written as an alternating word in its two generators $\widetilde{\mathbf{I}}_{+}$and $\widetilde{\mathbf{I}}_{-}$. By 5.26.iv both generators act antiholomorphically, and hence so does any word with an odd number of letters. By 5.26.ii any word in $\widetilde{\mathbf{D}}$ with an even number of letters lies in the cyclic subgroup $\widetilde{\mathbf{C}}=\left\langle\widetilde{\mathrm{T}}_{2 \widehat{\mathrm{p}}_{\tau}}\right\rangle \subset \mathrm{SU}(n)$ and hence $\widetilde{\mathbf{D}} \cap \operatorname{Isom}_{ \pm \mathrm{SL}}^{J}=\widetilde{\mathbf{C}}=\left\langle\widetilde{\mathrm{T}}_{2 \widehat{\mathrm{p}}_{\tau}}\right\rangle$ as claimed. The case $p>1$ and $p=q$ : Any nontrivial element in $\widetilde{\mathbf{D}}$ can be written as an alternating word in its two generators $\widetilde{\mathbf{I}}$ and $\widetilde{\mathbf{I}}_{+}$. An element of $\widetilde{\mathbf{D}}$ acts holomorphically if and only if it contains the antiholomorphic isometry $\widetilde{\mathbf{I}}_{+}$an even number of times. Hence a holomorphic isometry in $\widetilde{\mathbf{D}}$ has either (a) an even number of both generators or (b) an even number of $\widetilde{\underline{I}}_{+} \mathrm{s}$ and an odd number of $\widetilde{\underline{I}}_{\mathrm{s}}$. In case (b) any such element is equal to $\widetilde{\mathrm{T}}_{2 k \widehat{\mathrm{p}}_{\tau}} \circ \widetilde{\mathrm{I}}$ for some $k \in \mathbb{Z}$. In case (a), by 5.40 any such word is of the form $\widetilde{\mathrm{T}}_{2 k \widehat{\mathrm{p}}_{\tau}}$ for some $k \in \mathbb{Z}$. Hence $\widetilde{\mathbf{D}} \cap \operatorname{Isom}_{ \pm \mathrm{SL}}^{J}=\left\langle\widetilde{\mathbf{T}}, \widetilde{\mathrm{T}}_{2 \widehat{\mathrm{p}}_{\tau}}\right\rangle$ as claimed.

(iii) $p=1$ or $p>1$ and $p \neq q$ : $\widetilde{\mathbf{D}}$ centralizes $\widetilde{O}$ by 5.20 .ii and 5.26 .iii respectively. For the case $p>1$ and $p=q$ the result follows using 5.34.i,v and 5.26.iii. 
(iv) In all cases, we have $\widetilde{\mathbf{D}} \cap \widetilde{O} \subset \widetilde{\mathbf{D}} \cap \mathrm{O}(n) \subset \widetilde{\mathbf{D}} \cap \mathrm{SU}(n)^{ \pm}$and by part (ii) we have

$$
\widetilde{\mathbf{D}} \cap \operatorname{SU}(n)^{ \pm}= \begin{cases}\left\langle\widetilde{\mathbf{T}}_{2 \widehat{\mathbf{p}}_{\tau}}\right\rangle & \text { if } p=1 \text { or if } p>1 \text { and } p \neq q \\ \left\langle\widetilde{\mathbf{T}}, \widetilde{\mathbf{T}}_{2 \widehat{\mathbf{p}}_{\tau}}\right\rangle & \text { if } p>1 \text { and } p=q\end{cases}
$$

$p=1$ or $p>1$ and $p \neq q$ cases: Using the definitions of $\widetilde{\mathrm{T}}_{x}, \hat{\mathrm{T}}_{x}$ and $\widetilde{O} \subset \mathrm{O}(n) \subset \mathrm{U}(n)$ for $p=1$ we have

$$
\widetilde{\mathrm{T}}_{2 k \widehat{\mathrm{p}}_{\tau}} \in \widetilde{O} \Longleftrightarrow \hat{\mathrm{T}}_{2 k \widehat{\mathrm{p}}_{\tau}}=\left(\begin{array}{cc}
1 & 0 \\
0 & \pm 1
\end{array}\right)
$$

or if $p>1$ and $p \neq q$ (recall 3.3 for the definition of $\rho_{j k}$ )

$$
\widetilde{\mathrm{T}}_{2 k \widehat{\mathrm{p}}_{\tau}} \in \widetilde{O} \Longleftrightarrow \hat{\mathrm{T}}_{2 k \widehat{\mathrm{p}}_{\tau}}=\left(\begin{array}{cc} 
\pm 1 & 0 \\
0 & \pm 1
\end{array}\right)=\rho_{j k}, \quad \text { for some }(j, k) \in \mathbb{Z}_{2} \times \mathbb{Z}_{2} \text {. }
$$

Hence by $4.49 \widetilde{\mathbf{D}} \cap \widetilde{O} \neq(\mathrm{Id})$ if and only if $\mathbf{w}_{\tau}$ admits half-periods of type $(+-)$ when $p=1$ or if $\mathbf{w}_{\tau}$ admits strict-half periods when $p>1$ and $p \neq q$. If $p=1$ then by 4.49.iii $\mathbf{w}_{\tau}$ admits half-periods of type $(+-)$ if and only if $k_{0}$, the order of the rotational period, is even and the dimension $n$ is odd. In this case $\widetilde{\mathbf{D}} \cap \widetilde{O} \cong \mathbb{Z}_{2}$ where $\mathbb{Z}_{2}$ is the group generated by the involution $\widetilde{\mathrm{T}}_{k_{0} \widehat{\mathrm{p}}_{\tau}}$. If $p>1$ and $p \neq q$ then by $4.49 \mathbf{w}_{\tau}$ admits strict half-periods if and only if $k_{0}$ is even. Moreover, for fixed $p$ and $q$ only strict half-periods of type $(j k)$ occur where $j=q / \operatorname{hcf}(p, q)$ and $k=p / \operatorname{hcf}(p, q)$.

Case $p>1$ and $p=q$ : Written in block diagonal form (using (3.7) and (5.30)) $\widetilde{\mathrm{T}}_{2 k \widehat{\mathrm{p}}_{\tau}} \circ \underline{\mathrm{T}}$ is purely off-diagonal and hence not contained in $\widetilde{O}=$ $\mathrm{O}(p) \times \mathrm{O}(p) \subset \overline{\mathrm{O}}(2 \underset{\tau}{p})$ for any $k \in \mathbb{Z}$. Arguing as in the $p=1$ and $p \neq q$ cases we find that $\widetilde{\mathrm{T}}_{2 k \widehat{\mathrm{p}}_{\tau}} \in \widetilde{O}$ if and only if $\hat{\mathrm{T}}_{2 k \widehat{\mathrm{p}}_{\tau}}= \pm \mathrm{Id}$ and hence by 4.49 $\widetilde{\mathbf{D}} \cap \widetilde{O} \neq(\mathrm{Id})$ if and only if $\mathbf{w}_{\tau}$ admits strict half-periods of type $(--)$. Thus by $4.49 \widetilde{\mathbf{D}} \cap \widetilde{O}=(\mathrm{Id})$ if $k_{0}$ is infinite or odd. If $k_{0}$ is even then any odd multiple of $k_{0} \mathrm{p}_{\tau}$ is a strict half-period of type $(--)$. Therefore in this case, $\widetilde{\mathbf{D}} \cap \widetilde{O}=\left\langle\widetilde{\mathrm{T}}_{k_{0} \widehat{\mathrm{p}}_{\tau}}\right\rangle=\langle-\mathrm{Id}\rangle \cong \mathbb{Z}_{2}$.

Remark 5.41. By (5.37) any $\widetilde{\mathrm{M}} \in \widetilde{\operatorname{Sym}}\left(X_{\tau}\right)$ can be written (not uniquely though) in the form

$$
\widetilde{\mathrm{M}}=\widetilde{\gamma} \circ \widetilde{\mathrm{O}}
$$

for some $\widetilde{\gamma} \in \widetilde{\mathbf{D}}$ and $\widetilde{\mathrm{O}} \in \widetilde{O}$. Therefore $\widetilde{\operatorname{Sym}}\left(X_{\tau}\right) \subset \mathrm{Isom}_{ \pm \mathrm{SL}}$ (recall $\widetilde{\mathbf{D}} \subset$ Isom $_{ \pm \mathrm{SL}}$ and $\left.\widetilde{O} \subset \mathrm{O}(n) \subset \mathrm{SU}(n)^{ \pm} \subset \mathrm{Isom}_{ \pm \mathrm{SL}}\right)$. In particular, every element 
The geometry of $\mathrm{SO}(p) \times \mathrm{SO}(q)$-invariant special Lagrangian cones 221

of $\widetilde{\operatorname{Sym}}\left(X_{\tau}\right)$ acts on $\mathbb{C}^{n}$ either holomorphically or anti-holomorphically. The subgroup of holomorphic symmetries of $X_{\tau}$ is by 5.38.ii

$$
\widetilde{\operatorname{Sym}}\left(X_{\tau}\right) \cap \operatorname{SU}(n)^{ \pm}= \begin{cases}\left\langle\widetilde{\mathrm{T}}_{2 \widehat{\mathrm{p}}_{\tau}}\right\rangle \cdot \widetilde{O} & \text { if } p=1 \text { or } p>1 \text { and } p \neq q \\ \left\langle\widetilde{\mathbf{T}}, \widetilde{\mathrm{T}}_{2 \widehat{\mathrm{p}}_{\tau}}\right\rangle \cdot \widetilde{O} & \text { if } p>1 \text { and } p=q .\end{cases}
$$

As an immediate corollary of (5.37) and Lemma 5.38 we have the following result about the group structure of $\widetilde{\operatorname{Sym}}\left(X_{\tau}\right)$ :

Corollary 5.42. For $0<|\tau|<\tau_{\max }$ the structure of $\widetilde{\operatorname{Sym}}\left(X_{\tau}\right)$ is as follows:

(i) For $p=1$

(a) If $k_{0}$ is infinite or odd or the dimension $n$ is even, $\widetilde{\operatorname{Sym}}\left(X_{\tau}\right) \cong$ $\widetilde{\mathbf{D}} \times O(n-1)$.

(b) If $k_{0}$ is even and $n$ is odd, $\widetilde{\operatorname{Sym}}\left(X_{\tau}\right)$ is an internal central direct product of $\widetilde{\mathbf{D}}$ and $O(n-1)$ identifying the central subgroup $\widetilde{\mathbf{D}} \cap O(n-1) \cong \mathbb{Z}_{2}[5, p$. 29].

(ii) For $p>1$ and $p \neq q$ we have

(a) If $k_{0}$ is infinite or odd, $\widetilde{\operatorname{Sym}}\left(X_{\tau}\right) \cong \widetilde{\mathbf{D}} \times O(p) \times O(q)$.

(b) If $k_{0}$ is even, $\widetilde{\operatorname{Sym}}\left(X_{\tau}\right)$ is an internal central product of $\widetilde{\mathbf{D}}$ and $O(p) \times O(q)$ identifying the central subgroup $\widetilde{\mathbf{D}} \cap O(p) \times O(q) \cong \mathbb{Z}_{2}$.

(iii) For $p>1$ and $p=q$ if $k_{0}$ is odd then

$$
\widetilde{\operatorname{Sym}}\left(X_{\tau}\right) \cong O(p) \times O(p) \rtimes_{\lambda} \widetilde{\mathbf{D}}
$$

where the twisting homomorphism $\lambda: \widetilde{\mathbf{D}} \rightarrow$ Aut $O(p) \times O(p)$ is given by

$\lambda(\widetilde{\gamma})= \begin{cases}\mathrm{Id} & \text { if } \widetilde{\gamma} \in \widetilde{\mathbf{D}} \text { is a word containing an even number of copies of } \widetilde{\mathbf{T}}, \\ \mathbf{E}^{\prime} & \text { if } \widetilde{\gamma} \in \widetilde{\mathbf{D}} \text { is a word containing an odd number of copies of } \underline{\mathbf{T}},\end{cases}$ where $\mathrm{E}^{\prime}$ is the involution defined in (5.5).

Corollary 5.44. For $0<|\tau|<\tau_{\max }$ we have

$$
\operatorname{Per}\left(X_{\tau}\right)= \begin{cases}(\mathrm{Id}) & \text { if } k_{0} \text { is infinite; } \\ \left\langle\mathrm{T}_{2 k_{0} \mathbf{p}_{\tau}}\right\rangle & \text { if } k_{0} \text { is odd or } p=1 \text { and } n \text { is even; } \\ \left\langle\mathrm{T}_{k_{0} \mathbf{p}_{\tau}} \circ(-1)^{j} \operatorname{Id}_{\mathbb{S}^{p-1}} \circ(-1)^{k} \operatorname{Id}_{\mathbb{S}^{q-1}}\right\rangle & \text { if } k_{0} \text { is odd and } p>1 ; \\ \left\langle\mathrm{T}_{k_{0} \mathbf{p}_{\tau}} \circ-\operatorname{Id}_{\mathbb{S}^{n-1}}\right\rangle & \text { if } k_{0} \text { is even, } p=1 \text { and } n \text { is odd; }\end{cases}
$$

where $j=q / \operatorname{hcf}(p, q)$ and $k=p / \operatorname{hcf}(p, q)$. 
Proof. This follows from the results on the structure of $\operatorname{Sym}\left(X_{\tau}\right)$ and $\widetilde{\operatorname{Sym}}\left(X_{\tau}\right)$ proved in this section together with the fact that $\operatorname{Per}\left(X_{\tau}\right)=\operatorname{ker} \widetilde{\rho}$ where $\widetilde{\rho}: \operatorname{Sym}\left(X_{\tau}\right) \rightarrow \widehat{\operatorname{Sym}}\left(X_{\tau}\right)$ is the homomorphism described in 2.10.

\section{Geometric features of $\boldsymbol{X}_{\tau}$}

This section describes various geometric features of $X_{\tau}$ with particular emphasis on its geometry as $\tau \rightarrow 0$, the action of $\operatorname{Sym}\left(X_{\tau}\right)$ on various subdomains of $\mathrm{Cyl}^{p, q}$ and the action of $\widetilde{\operatorname{Sym}}\left(X_{\tau}\right)$ on various equatorial spheres associated with $X_{\tau}$.

Waists, bulges and approximating spheres. In this section we describe distinguished subsets of $\mathrm{Cyl}^{p, q}$ called the waists and bulges of $X_{\tau}$ and describe the action of $\operatorname{Sym}\left(X_{\tau}\right)$ on these subsets. We also attach to each bulge a $p+q-1$ dimensional equatorial subsphere of $\mathbb{S}^{2(p+q)-1}$, called the approximating sphere of the bulge and describe symmetries associated with these approximating spheres. The terminology approximating sphere is justified by (6.21) where we show that for $\tau$ sufficiently close to 0 the image of each bulge under $X_{\tau}$ is close to its approximating sphere.

Fix admissible integers $p$ and $q$ and let $X_{\tau}: \mathrm{Cyl}^{p, q} \rightarrow \mathbb{S}^{2(p+q)-1}$ be the 1-parameter family of $\mathrm{SO}(p) \times \mathrm{SO}(q)$-equivariant special Legendrian immersions defined in (3.29) and $g_{\tau}$ denote the pullback metric on $\mathrm{Cyl}^{p, q}$ induced by $X_{\tau}$. Throughout this section, we assume that $|\tau|<\tau_{\max }$.

Definition 6.1. A waist of $\left(\mathrm{Cyl}^{p, q}, g_{\tau}\right)$ is a meridian $\{t\} \times \mathrm{Mer}^{p, q}$ of $\mathrm{Cyl}{ }^{p, q}$ on which the radius of one spherical factor of the meridian is minimal.

Waists for $p=1$. If $p=1$ then a waist is any meridian $\{t\} \times \mathbb{S}^{n-2}$ such that $y_{\tau}(t)=y_{\min }$. Recall from (4.5) that our choice of initial conditions for $\mathbf{w}_{\tau}$ in the case $p=1$ forces $y_{\tau}$ to have a maximum at $t=0$ and a minimum at $t=\mathrm{p}_{\tau}$. Hence using the symmetries of $y_{\tau}$ described in (4.4), $y_{\tau}$ has maxima at precisely $2 k \mathrm{p}_{\tau}$ and minima at precisely $(2 k+1) \mathrm{p}_{\tau}$ for each $k \in \mathbb{Z}$. See figure 1 for an illustration. Therefore the meridian $\{t\} \times \mathbb{S}^{n-2}$ is a waist of $X_{\tau}$ if and only if $t \in(2 \mathbb{Z}+1) \mathrm{p}_{\tau}$. For any $k \in \mathbb{Z}$ we define the $k$ th waist $W[k]$ of $\mathrm{Cyl}^{1, n-1}$ to be

$$
W[k]=\left\{(2 k-1) \mathbf{p}_{\tau}\right\} \times \mathbb{S}^{n-2} .
$$

Waists for $p>1$. If $p>1$ then a waist is any meridian $\{t\} \times \operatorname{Mer}^{p, q}$ such that either $y_{\tau}(t)=y_{\min }$ or $y_{\tau}(t)=y_{\max }$; we call a waist on which $y(t)=y_{\max }$ 
The geometry of $\mathrm{SO}(p) \times \mathrm{SO}(q)$-invariant special Lagrangian cones 223

a waist of type 1 , since it is the radius of the first spherical factor $\mathbb{S}^{p-1}$ which is minimal on such a waist. Similarly, a waist on which $y_{\tau}(t)=y_{\min }$ is called a waist of type 2 , since the radius of the second spherical factor $\mathbb{S}^{q-1}$ is minimal on such a waist. Recall from (4.1) that $y_{\tau}$ attains a maximum at $t=-\mathbf{p}_{\tau}^{-}$, a minimum at $t=\mathrm{p}_{\tau}^{+}$, is decreasing on $\left(-\mathbf{p}_{\tau}^{-}, \mathbf{p}_{\tau}^{+}\right)$and increasing on $\left(\mathrm{p}_{\tau}^{+}, \mathrm{p}_{\tau}+\mathrm{p}_{\tau}^{+}\right)$- see figure 2 . Hence $\{t\} \times \mathrm{Mer}^{p, q}$ is a waist of type 1 if and only if $t+\mathrm{p}_{\tau}^{-} \in 2 \mathrm{p}_{\tau} \mathbb{Z}$ and a waist of type 2 if and only if $t-\mathrm{p}_{\tau}^{+} \in 2 \mathrm{p}_{\tau} \mathbb{Z}$. For any $k \in \mathbb{Z}$, we define the $k$ th waist $W[k]$ of $\mathrm{Cyl}^{p, q}$ by

$$
\begin{aligned}
W[2 l+1]:=\left\{2 l \mathbf{p}_{\tau}+\mathbf{p}_{\tau}^{+}\right\} \times \operatorname{Mer}^{p, q} & \text { if } k=2 l+1 \text { some } l \in \mathbb{Z} \\
W[2 l]:=\left\{2 l \mathbf{p}_{\tau}-\mathbf{p}_{\tau}^{-}\right\} \times \operatorname{Mer}^{p, q} & \text { if } k=2 l \quad \text { some } l \in \mathbb{Z} .
\end{aligned}
$$

The action of $\operatorname{Isom}\left(\mathrm{Cyl}^{p, q}, g_{\tau}\right)$ on waists. Any element of $\operatorname{Isom}\left(\mathrm{Cyl}{ }^{p, q}, g_{\tau}\right)$ permutes the waists of $\mathrm{Cy}^{p, q}$. If $p=1$ then all waists are isometric and $\operatorname{Isom}\left(\mathrm{Cyl}^{1, n-1}, g_{\tau}\right)$ acts transitively on the set of waists. If $p>1$ and $p \neq q$ then waists of type 1 and type 2 are not isometric and therefore $\operatorname{Isom}\left(\mathrm{Cyl}^{p, q}, g_{\tau}\right)$ cannot act transitively on the set of all waists. However, all waists of fixed type are isometric and $\operatorname{Isom}\left(\mathrm{Cyl}^{p, q}, g_{\tau}\right)$ acts transitively on the set of waists of fixed type.

If $p>1$ and $p=q$, waists of type 1 and type 2 are isometric (recall (4.11)). Recall from 5.6.iii that $\operatorname{Isom}\left(\mathrm{Cyl}^{p, p}, g_{\tau}\right)=\mathbf{D} \cdot \mathrm{O}(p) \times \mathrm{O}(p)$ where $\mathbf{D}=\left\langle\underline{T} \circ \mathbf{E}, \underline{T}_{p_{\tau} / 2}\right\rangle$. Isometries containing an even number of copies of $\underline{T} \circ \mathbf{E}$ preserve the type of any waist, while isometries containing an odd number of copies of $I \circ E$ exchange the two types of waist. The full group $\operatorname{Isom}\left(\mathrm{Cyl}^{p, p}, g_{\tau}\right)$ acts transitively on the set of all waists.

Bulges. The set of all waists $W$ of $\left(\mathrm{Cyl}^{p, q}, g_{\tau}\right)$ is a hypersurface with countably many components $W[k](k \in \mathbb{Z})$ and the complement of $W$ in $\mathrm{Cyl}^{p, q}$ has countably many components.

Definition 6.4. A bulge of $\left(\mathrm{Cyl}^{p, q}, g_{\tau}\right)$ is a connected component of $\left(\mathrm{Cyl}^{p, q} \backslash W\right)$. For any $k \in \mathbb{Z}$ the $k$ th bulge $\hat{S}[k]$ of $\left(\mathrm{Cyl}^{p, q}, g_{\tau}\right)$ is the unique connected component of $\left(\mathrm{Cyl}^{p, q} \backslash W\right)$ such that $\partial \hat{S}[k]=W[k] \cup W[k+1]$. We call $W[k]$ and $W[k+1]$ the boundary waists of the bulge $\hat{S}[k]$.

More concretely, for $p=1$ the $k$ th bulge of $\mathrm{Cyl}^{1, n-1}$ is

$$
\hat{S}[k]=\left((2 k-1) \mathbf{p}_{\tau},(2 k+1) \mathbf{p}_{\tau}\right) \times \mathbb{S}^{n-2}=\mathrm{T}_{2 k \mathbf{p}_{\tau}} \hat{S}[0],
$$


while for $p>1$ the $k$ th bulge of $\mathrm{Cyl}^{p, q}$ is

$$
\hat{S}[2 l]=\left(-\mathrm{p}_{\tau}^{-}+2 l \mathrm{p}_{\tau},-\mathrm{p}_{\tau}^{-}+(2 l+1) \mathrm{p}_{\tau}\right) \times \operatorname{Mer}^{p, q}=\mathrm{T}_{2 l \mathrm{p}_{\tau}} \hat{S}[0] \quad \text { if } k=2 l ;
$$

$\hat{S}[2 l+1]=\left(\mathrm{p}_{\tau}^{+}+2 l \mathrm{p}_{\tau}, \mathrm{p}_{\tau}^{+}+(2 l+1) \mathrm{p}_{\tau}\right) \times \operatorname{Mer}^{p, q}=\mathrm{T}_{2 l \mathrm{p}_{\tau}} \hat{S}[1] \quad$ if $k=2 l+1$.

Since any isometry in $\operatorname{Isom}\left(\mathrm{Cyl}^{p, q}, g_{\tau}\right)=\operatorname{Sym}\left(X_{\tau}\right)$ permutes the waists of $\left(\mathrm{Cyl}^{p, q}, g_{\tau}\right)$ it also permutes the bulges of $\mathrm{Cyl}^{p, q}$. Moreover $\mathrm{Isom}\left(\mathrm{Cyl}{ }^{p, q}, g_{\tau}\right)$ acts transitively on the set of all bulges.

Definition 6.7. For any $k \in \mathbb{Z}$ we define $\operatorname{Sym}_{k}\left(X_{\tau}\right)$ to be the subgroup of $\operatorname{Sym}\left(X_{\tau}\right)=\operatorname{Isom}\left(\mathrm{Cyl}^{p, q}, g_{\tau}\right)$ leaving the $k$ th bulge $\hat{S}[k]$ invariant.

Since $\operatorname{Isom}\left(\mathrm{Cyl}^{p, q}, g_{\tau}\right)=\operatorname{Sym}\left(X_{\tau}\right)$ acts transitively on the set of all bulges the subgroups $\operatorname{Sym}_{k}\left(X_{\tau}\right)$ are all conjugate in $\operatorname{Sym}\left(X_{\tau}\right)$. In particular, they are all isomorphic as groups.

Lemma 6.8 (Structure of $\operatorname{Sym}_{k}\left(X_{\tau}\right)$; cf. Lemma 6.13). For any fixed $k \in \mathbb{Z}$ we have

$$
\begin{aligned}
& \operatorname{Sym}_{k}\left(X_{\tau}\right) \\
& \quad= \begin{cases}\left\langle\underline{\mathrm{T}}_{2 k \mathrm{p}_{\tau}}\right\rangle \cdot O(n-1) \cong O(1) \times O(n-1) & \text { if } p=1 ; \\
O(p) \times O(q) & \text { if } p>1 \text { and } p \neq q ; \\
\left\langle\underline{\mathrm{T}}_{k \mathrm{p}_{\tau}} \circ \mathrm{E}\right\rangle \cdot O(p) \times O(p) \cong O(p) \times O(p) \rtimes \mathbb{Z}_{2} & \text { if } p>1 \text { and } p=q .\end{cases}
\end{aligned}
$$

Proof. An element of $\operatorname{Sym}\left(X_{\tau}\right)$ belongs to $\operatorname{Sym}_{k}\left(X_{\tau}\right)$ if and only if it leaves invariant the union of the two boundary waists $W[k]$ and $W[k+1]$. The lemma now follows using the structure of $\operatorname{Isom}\left(\mathrm{Cyl}^{p, q}, g_{\tau}\right)=\operatorname{Sym}\left(X_{\tau}\right)$ established in 5.6 to determine its action on the set of waists $W$.

$(p, q)$-marked special Legendrian spheres and approximating spheres. Fix admissible integers $p$ and $q$. We now define the important concept of a $(p, q)$ marked special Legendrian sphere. We will see shortly that we can associate a $(p, q)$-marked SL sphere $\mathbb{S}[k]$ to every bulge of $X_{\tau}: \mathrm{Cyl}^{p, q} \rightarrow \mathbb{S}^{2(p+q)-1}$. Moreover, for $\tau$ sufficiently small the image of the $k$ th bulge $\hat{S}[k]$ under $X_{\tau}$ is close to the marked SL sphere $\mathbb{S}[k]$.

Definition 6.9. If $(p, q)=(1, n-1)$ then a $(p, q)$-marked $S L$ sphere is a pair $\{ \pm e, \mathbb{S}\}$ consisting of an equatorial $n-1$ sphere $\mathbb{S}$ of $\mathbb{S}^{2 n-1}$ which is special Legendrian (for the correct orientation) and a pair of antipodal points 
The geometry of $\mathrm{SO}(p) \times \mathrm{SO}(q)$-invariant special Lagrangian cones 225

$\pm e \in \mathbb{S}$. We call $\pm e \subset \mathbb{S}$ the attachment set (or alternatively the marked set) of the $(1, n-1)$-marked SL sphere $( \pm e, \mathbb{S})$.

If $p>1$ then a $(p, q)$-marked $S L$ sphere is a triple consisting of an equatorial special Legendrian (for the correct orientation) $p+q-1$ sphere $\mathbb{S}$ of $\mathbb{S}^{2(p+q)-1}$, an equatorial subsphere $\mathbb{S}_{p-1} \subset \mathbb{S}$ of dimension $p-1$ and the orthogonal equatorial subsphere $\mathbb{S}_{q-1} \subset \mathbb{S}$. We call $\mathbb{S}_{p-1} \cup \mathbb{S}_{q-1} \subset \mathbb{S}$ the attachment set or marked set of the $(p, q)$-marked SL sphere $\left(\mathbb{S}_{p-1}, \mathbb{S}_{q-1}, \mathbb{S}\right)$.

A $(1, n-1)$-marked SL sphere is equivalent to a pair $\left\{l, \Pi_{n}\right\}$ where $\Pi_{n} \subset \mathbb{C}^{n}$ is a SL $n$-plane and $l=\langle \pm e\rangle \subset \Pi_{n}$ is an unoriented real line in the $n$-plane $\Pi_{n}$. We call $\mathbb{S}^{n-1} \subset \mathbb{R}^{n} \subset \mathbb{C}^{n}$ the standard special Legendrian sphere and $\left\{ \pm e_{1}, \mathbb{S}^{n-1}\right\}$ the standard $(1, n-1)$-marked special Legendrian sphere, where $e_{1}, \ldots, e_{n}$ is the standard oriented orthonormal basis of $\mathbb{R}^{n}$.

For $p>1$ a $(p, q)$-marked SL sphere is equivalent to a triple $\left\{\Pi_{p}, \Pi_{q}=\Pi_{p}^{\perp}, \Pi_{p+q}\right\}$ where $\Pi_{p+q} \subset \mathbb{C}^{p+q}$ is a SL $p+q$-plane and $\Pi_{p} \subset \Pi_{p+q}$ is a real $p$-plane in $\Pi_{p+q}$ and $\Pi_{q}=\Pi_{p}^{\perp} \subset \Pi_{p+q}$. The $(p, q)$-marked SL sphere with $\Pi_{p+q}=\mathbb{R}^{p+q} \subset \mathbb{C}^{p+q}, \Pi_{p}=\mathbb{R}^{p} \times\{0\} \subset \Pi_{p+q}$ and $\Pi_{q}=\{0\} \times \mathbb{R}^{q}=$ $\Pi_{p}^{\perp} \subset \Pi_{p+q}$ we call the standard $(p, q)$-marked $S L$ sphere. The choice of a real $p$-plane $\Pi_{p} \subset \Pi_{p+q}$ determines the $q$-plane $\Pi_{q}$ as the orthogonal complement of $\Pi_{p}$ inside $\Pi_{p+q}$.

Fix admissible integers $p$ and $q$ and $\tau$ satisfying $|\tau|<\tau_{\max }$. To each bulge $\hat{S}[k]$ of $X_{\tau}$ we now associate a $(p, q)$-marked SL sphere called its approximating (marked) sphere $\mathbb{S}[k]$.

Definition 6.10. For each $k \in \mathbb{Z}$ we define a $(p, q)$-marked sphere $\mathbb{S}[k]$ as follows. For $k=0$ we define $\mathbb{S}[0]$ to be the standard $(p, q)$-marked SL sphere defined following 6.9.

For $p=1$ we define

$$
\mathbb{S}[k]:=\widetilde{\mathrm{T}}_{2 k \widehat{\mathrm{p}}_{\tau}} \mathbb{S}[0] \quad \text { if } k \neq 0 .
$$

For $p>1$ we define

$$
\mathbb{S}[k]:= \begin{cases}\widetilde{\mathrm{T}}_{2 l \widehat{\mathfrak{p}}_{\tau}} \mathbb{S}[0] & \text { if } k=2 l ; \\ \widetilde{\mathrm{T}}_{2 l \widehat{\mathbf{p}}_{\tau}} \circ \widetilde{\mathbf{T}}_{+} \mathbb{S}[0] & \text { if } k=2 l+1 .\end{cases}
$$

$\mathbb{S}[k]$ is called the approximating $(p, q)$-marked sphere (or more simply the approximating sphere) associated with the $k$ th bulge $\hat{S}[k]$ of $X_{\tau}$. 
Note that since $\widetilde{\mathrm{T}}_{x} \in \mathrm{SU}(n) \subset$ Isom $_{\mathrm{SL}}$ for all $x$, for $p=1$ if we orient the central marked sphere $\mathbb{S}[0]$ so that it is special Legendrian then the orientation $\mathbb{S}[k]=\widetilde{\mathbf{T}}_{2 k \widehat{p}_{\tau}} \mathbb{S}[0]$ inherits from $\mathbb{S}[0]$ via $\widetilde{\mathbf{T}}_{2 k \widehat{\mathrm{p}}_{\tau}}$ also makes $\mathbb{S}[0]$ special Legendrian. However, for $p>1$ recall from 5.26 that $\widetilde{\mathbf{T}}_{+} \in \operatorname{Isom}_{ \pm \mathrm{SL}} \backslash \mathrm{Isom}_{\mathrm{SL}}$; this occurs because the corresponding symmetry $\underline{\mathbf{T}}_{\mathrm{p}_{\tau}^{+}} \in \operatorname{Sym}\left(X_{\tau}\right) \subset \operatorname{Diff}$ $\left(\mathrm{Cyl}^{p, q}\right)$ reverses orientation on $\mathrm{Cyl}^{p, q}$. Hence if we orient the central marked sphere $\mathbb{S}[0]$ so that it is special Legendrian then the orientation inherited on any odd approximating sphere $\mathbb{S}[2 l+1]=\widetilde{\mathbf{T}}_{2 l \widehat{\mathbf{p}}_{\tau}} \circ \widetilde{\mathbf{T}}_{+} \mathbb{S}[0]$ from $\mathbb{S}[0]$ makes it anti-special Legendrian.

Lemma 6.13 (Action of $\widetilde{\operatorname{Sym}}\left(X_{\tau}\right)$ on the approximating spheres; cf. 6.8). $\widetilde{\operatorname{Sym}}\left(X_{\tau}\right)=\widetilde{\mathbf{D}} \cdot \widetilde{O}$ acts on the approximating marked spheres of $X_{\tau}$ as follows:

(i) For any $p$ and $q$ we have $\widetilde{O} \mathbb{S}[k]=\mathbb{S}[k]$ for all $k \in \mathbb{Z}$ and all $\widetilde{\mathrm{O}} \in \widetilde{O}$.

(ii) For $p=1$ and any $k \in \mathbb{Z}, \widetilde{\mathbf{D}}=\left\langle\underline{\widetilde{T}}, \widetilde{\mathbf{T}}_{2 \widehat{\mathrm{p}}_{\tau}}\right\rangle$ acts by

$$
\begin{aligned}
\widetilde{\mathbf{T}}_{2 \widehat{\mathrm{p}}_{\tau}} \mathbb{S}[k] & =\mathbb{S}[k+1] ; \\
\widetilde{\mathbf{T}} \mathbb{S}[k] & =\mathbb{S}[-k] .
\end{aligned}
$$

(iii) For $p>1$ and $p \neq q$, for any $k \in \mathbb{Z} \widetilde{\mathbf{D}}=\left\langle\underline{\mathbf{T}}_{+}, \underline{\mathbf{I}}_{-}\right\rangle$acts by

$$
\begin{aligned}
& \underline{\widetilde{T}}_{+} \mathbb{S}[k]=\mathbb{S}[1-k] ; \\
& \underline{\widetilde{T}}_{-} \mathbb{S}[k]=\mathbb{S}[-1-k] .
\end{aligned}
$$

(iv) For $p>1$ and $p=q$, for any $k \in \mathbb{Z}, \widetilde{\mathbf{D}}=\left\langle\underline{\widetilde{\mathbf{T}}}, \underline{\mathbf{I}}_{+}\right\rangle$acts by

$$
\begin{aligned}
\widetilde{\mathbf{T}}_{+} \mathbb{S}[k] & =\mathbb{S}[1-k] ; \\
\widetilde{\mathbf{T}} \mathbb{S}[k] & =\mathbb{S}[-k] .
\end{aligned}
$$

Proof. The lemma follows using 6.10, the commutation properties between $\widetilde{\mathbf{D}}$ and $\widetilde{O}$ given in (5.37).iii, the structure of the group $\widetilde{\mathbf{D}}$ given in 5.38.i, and the action of $\widetilde{\mathbf{D}}$ and $\widetilde{O}$ on $\mathbb{S}[0]$. We leave the details to the reader.

Remark 6.17. For $p>1$, it follows immediately from 6.15 and 5.26.ii that for any $k \in \mathbb{Z}$

$$
\widetilde{\mathrm{T}}_{2 \widehat{\mathrm{p}}_{\tau}} \mathbb{S}[k]=\underline{\widetilde{T}}_{+} \circ \underline{\mathbf{I}}_{-} \mathbb{S}[k]=\mathbb{S}[k+2],
$$


The geometry of $\mathrm{SO}(p) \times \mathrm{SO}(q)$-invariant special Lagrangian cones 227

i.e., the rotational period $\widetilde{\mathrm{T}}_{2 \widehat{\mathrm{p}}_{\tau}}$ maps $\mathbb{S}[k] \mapsto \mathbb{S}[k+2]$ unlike the case $p=1$ (recall 6.14a) where it maps $\mathbb{S}[k] \mapsto \mathbb{S}[k+1]$. This reflects a fundamental difference in the geometry of $X_{\tau}$ in the cases $p=1$ and $p>1$.

Corollary 6.19. $\widetilde{\operatorname{Sym}}_{k}\left(X_{\tau}\right)$, the subgroup of $\widetilde{\operatorname{Sym}}\left(X_{\tau}\right)$ leaving the kth approximating sphere $\mathbb{S}[k]$ invariant is

$$
\widetilde{\operatorname{Sym}}_{k}\left(X_{\tau}\right)= \begin{cases}\left\langle\widetilde{\mathbf{T}}_{2 k \widehat{\mathrm{p}}_{\tau}}\right\rangle \cdot O(n-1) & \text { if } p=1 \\ O(p) \times O(q) & \text { if } p>1 \text { and } p \neq q \\ \left\langle\widetilde{\mathbf{T}}_{k \widehat{\mathrm{p}}_{\tau}}\right\rangle \cdot O(p) \times O(p) & \text { if } p>1 \text { and } p=q\end{cases}
$$

It follows from 6.8 and 6.13 together with 5.15, 5.22 and 5.28 that $\widetilde{\operatorname{Sym}}_{k}\left(X_{\tau}\right)=\widetilde{\rho}\left(\operatorname{Sym}_{k}\left(X_{\tau}\right)\right)$ where $\widetilde{\rho}: \operatorname{Sym}\left(X_{\tau}\right) \rightarrow \widetilde{\operatorname{Sym}}\left(X_{\tau}\right)$ is the homomorphism defined in 2.10 and $\operatorname{Sym}_{k}\left(X_{\tau}\right)$ is defined in 6.7.

The limiting geometry of $\boldsymbol{X}_{\boldsymbol{\tau}}$ as $\boldsymbol{\tau} \rightarrow \mathbf{0}$. Next we describe the geometry of $X_{\tau}$ as $\tau \rightarrow 0$ concentrating on the almost spherical regions of $X_{\tau}$ that asymptotically resemble equatorial spheres and on the necks which asymptotically resemble small Lagrangian catenoids or the product of a unit sphere with a small Lagrangian catenoid. The fact that $X_{\tau}$ degenerates to a union of very simple geometric objects is fundamental to our gluing constructions in $[9-12]$.

Almost spherical regions of $\boldsymbol{X}_{\boldsymbol{\tau}}$ and approximating spheres. Recall that by $(3.23) \mathbf{w}_{\tau}$ depends analytically on $\tau \in\left(-\tau_{\max }, \tau_{\max }\right)$ and the image of $\mathbf{w}_{0}$ is contained in $\mathbb{S}^{1} \subset \mathbb{R}^{2} \subset \mathbb{C}^{2}$. This implies that $X_{\tau}$ depends analytically on $\tau$ and that $X_{0}$ gives a parametrization of $\mathbb{S}[0] \backslash \mathbb{M}[0]$ where $\mathbb{S}[0]$ denotes the standard $(p, q)$-marked special Legendrian sphere (recall 6.10) and $\mathbb{M}[0]$ is its marked set (two orthogonal equators of dimension $p-1$ and $q-1$ if $p>1$ or two antipodal points if $p=1$ ).

Because of the analytic dependence on $\tau, X_{\tau}$ smoothly converges to $X_{0}$ as $\tau \rightarrow 0$ on any compact subset $K \subset \subset \mathrm{Cyl}^{p, q}$. Define

$$
S[0]:=[-b, b] \times \operatorname{Mer}^{p, q},
$$

then by choosing $b \in \mathbb{R}^{+}$sufficiently large we can ensure the image $X_{0}(S[0])$ contains any given compact subset of $\mathbb{S}[0] \backslash \mathbb{M}[0]$. If $|\tau|$ is small enough in 
terms of $b$, then $\mathbf{w}_{\tau}$ and therefore $X_{\tau}$ satisfy

$$
\begin{gathered}
\left\|\mathbf{w}_{\tau}-\mathbf{w}_{0}: C^{k}([-b, b])\right\| \leq C(b, k)|\tau| \\
\left\|X_{\tau}-X_{0}: C^{k}(S[0])\right\| \leq C(b, k)|\tau|
\end{gathered}
$$

where we can use the standard metric of the cylinder to define the $C^{k}$ norm and the constant $C(b, k)$ depends only on $b$ and $k$. This motivates us to call $S[0]$ an almost spherical region of $X_{\tau}$. Note that the definition of $S[0]$ depends on a choice of $b$ which we do not make precise here, but which is supposed to be chosen large enough as needed. The freedom to choose an appropriate $b$ to define the almost spherical regions is needed in our gluing constructions [9-12]. (6.21) implies that for $\tau$ sufficiently small the image under $X_{\tau}$ of the almost spherical region $S[0] \subset \subset \hat{S}[0]$ is close to its approximating sphere $\mathbb{S}[0]$ and converges as $\tau \rightarrow 0$ to a fixed compact subset of $\mathbb{S}[0] \backslash \mathbb{M}[0]$ (depending on the choice of $b$ ). This explains the origin of the terminology approximating sphere and also one of the roles played by the marked set.

If we have fixed the almost spherical region $S[0]$ as above, then we can mimic the definition of $\hat{S}[k]$ in terms of $\hat{S}[0]$ (recall $6.5,6.6$ ) to define the $k$ th almost spherical region $S[k] \subset \subset \hat{S}[k] \subset \mathrm{Cyl}^{p, q}$ of $X_{\tau}$ in terms of $S[0]$ by

$$
S[k]:= \begin{cases}\mathrm{T}_{2 k \mathbf{p}_{\tau}} S[0] & \text { if } p=1 \\ \mathrm{~T}_{2 l \mathbf{p}_{\tau}} S[0] & \text { if } p>1 \text { and } k=2 l \\ \mathrm{~T}_{2 l \mathbf{p}_{\tau}} \circ \underline{\mathrm{T}}_{\mathbf{p}_{\tau}^{+}} S[0] & \text { if } p>1 \text { and } k=2 l+1 .\end{cases}
$$

Because $\mathrm{p}_{\tau} \rightarrow \infty$ when $p=1$ and $\mathrm{p}_{\tau}^{+}, \mathrm{p}_{\tau}^{-} \rightarrow \infty$ as $\tau \rightarrow 0$ (see 7.3 for a precise statement), every almost spherical region $S[k]$ with $k \neq 0$ "slides off the end" of $\mathrm{Cyl}^{p, q}$ as $\tau \rightarrow 0$. However, by using an element of $\mathrm{Isom}\left(\mathrm{Cyl}^{p, q}, g_{\tau}\right)$ to bring $S[k]$ back to $S[0]$ we can also infer the small $\tau$ behaviour of $X_{\tau}$ on the other almost spherical regions $S[k]$. Using the relevant symmetries of $X_{\tau}$ (from 5.15 and 5.22) we see that on the $k$ th almost spherical region $S[k] \subset \subset \hat{S}[k], X_{\tau}$ satisfies the analogue of (6.21) with $X_{0}$ replaced by the embedding $X[k]: \mathrm{Cyl}^{p, q} \rightarrow \mathbb{S}^{2(p+q)-1}$ defined as

$$
X[k]:= \begin{cases}\widetilde{\mathrm{T}}_{2 k \widehat{\mathbf{p}}_{\tau}} \circ X_{0} \circ \mathrm{T}_{-2 k \mathbf{p}_{\tau}} & \text { if } p=1 ; \\ \widetilde{\mathrm{T}}_{2 l \widehat{\mathbf{p}}_{\tau}} \circ X_{0} \circ \mathrm{T}_{-2 l \mathbf{p}_{\tau}} & \text { if } p>1 \text { and } k=2 l \\ \widetilde{\mathbf{T}}_{2 l \widehat{\mathbf{p}}_{\tau}} \circ \underline{\mathbf{T}}_{+} \circ X_{0} \circ \underline{\mathbf{T}}_{\mathbf{p}_{\tau}^{+}} \circ \mathrm{T}_{-2 l \mathbf{p}_{\tau}} & \text { if } p>1 \text { and } k=2 l+1 .\end{cases}
$$

For $k \neq 0 \quad X[k]$ itself depends on $\tau$. The image of $\mathrm{Cyl}^{p, q}$ under $X[k]$ is $\mathbb{S}[k] \backslash \mathbb{M}[k]$, where $\mathbb{S}[k]$ denotes the $k$ th $(p, q)$-marked approximating sphere 
The geometry of $\mathrm{SO}(p) \times \mathrm{SO}(q)$-invariant special Lagrangian cones 229

and $\mathbb{M}[k]$ is its marked set, both of which also depend on $\tau$ for $k \neq 0$. Nevertheless, we have that for $\tau$ sufficiently small the image of the almost spherical region $S[k] \subset \subset \hat{S}[k]$ under $X_{\tau}$ is close to its approximating sphere $\mathbb{S}[k]$.

Each almost spherical region $S[k]$ of $\mathrm{Cyl}^{p, q}$ connects to its neighbouring almost spherical regions $S[k-1]$ and $S[k+1]$ in the two adjacent bulges $\hat{S}[k-1]$ and $\hat{S}[k+1]$ via a pair of transition regions whose images under $X_{\tau}$ for $\tau$ sufficiently small localize near the two components of the marked set $\mathbb{M}[k]$ of the $k$ th approximating marked sphere $\mathbb{S}[k]$. This pair of transition regions is centred on the two boundary waists $W[k]$ and $W[k+1]$ of $\hat{S}[k]$ (recall 6.4). In the next section we study the geometry of $X_{\tau}$ in the vicinity of the waists as $\tau \rightarrow 0$.

Waists, necks and Lagrangian catenoids. Recall from (6.1) that a waist $W[k]$ is a meridian of $\mathrm{Cyl}^{p, q}$ on which the radius of one spherical factor of the meridian is minimal. The vicinity of any meridian we call a neck. The necks are the regions of $\left(\mathrm{Cyl}^{p, q}, g_{\tau}\right)$ where the magnitude of the curvature is largest and where as $\tau \rightarrow 0$ the curvature becomes unbounded. We will show below that for $\tau$ sufficiently small any neck of $X_{\tau}$-appropriately scaled and repositioned - is a small perturbation of a (truncated) Lagrangian catenoid (see 6.24) in the case $p=1$, or the product of a large round sphere with a Lagrangian catenoid in the case $p>1$. This is reminiscent of the Delaunay surfaces [17, Lemma A.2.1] whose highly curved regions approximate a scaled repositioned catenoid.

The Lagrangian catenoid. We give a brief description of the Lagrangian catenoid in $\mathbb{C}^{n}$ suited to our present purposes, stating a number of basic properties without proof.

Let $w: I \rightarrow \mathbb{C}^{*}$ be a smooth immersed curve. Consider the ODEs

$$
\dot{w}=\bar{w}^{n-1} .
$$

(6.22) can be viewed as a degenerate analogue of (3.8): see (6.30) and (6.37). The ODE (6.22) admits the conserved quantity $\mathcal{I}:=\operatorname{Im} w^{n}$, cf. $\mathcal{I}_{2}$ defined in (3.8.i).

The case $n=2$ is special because (6.22) reduces to a linear ODE. Straightforward calculation shows that any solution to this linear ODE has the form

$$
w(t)=a e^{t}+i b e^{-t}, \quad \text { for } a, b \in \mathbb{R},
$$

and that $\operatorname{Im} w^{2}(t) \equiv 2 a b$. In particular, when $n=2$ all solutions of (6.22) are defined for all $t \in \mathbb{R}$ (in contrast to the case when $n>2$ ). 
For $n>2$ one can show that the initial value problem for $(6.22)$ with any initial data $w(0) \in \mathbb{C}^{*}$ has a unique real analytic solution $w: J \rightarrow \mathbb{C}^{*}$ defined on a bounded interval $J \subset \mathbb{R}$. Let $w_{1}$ denote the unique solution of (6.22) with initial data $w(0)=\mathrm{e}^{\mathrm{i} \pi / 2 n}$ (and hence $\left.\mathcal{I}\left(w_{1}\right)=1\right)$. Then $w_{1}$ has the symmetry $w_{1} \circ \underline{\mathrm{T}}=\mathrm{e}^{-\mathrm{i} \pi / n} \bar{w}_{1}$, where $\underline{\mathrm{I}}$ denotes reflection in the origin $t \mapsto-t$. The solution $w_{1}$ is defined on the time interval $I=(-T, T)$ where

$$
T:=\int_{1}^{\infty} \frac{d y}{2 \sqrt{y^{n}-1}}=\frac{\Gamma\left(\frac{1}{2}\right) \Gamma\left(\frac{1}{2}-\frac{1}{n}\right)}{2 \Gamma\left(-\frac{1}{n}\right)}=\frac{\sqrt{\pi} \Gamma\left(\frac{1}{2}-\frac{1}{n}\right)}{2 \Gamma\left(-\frac{1}{n}\right)},
$$

and $\Gamma$ denotes the gamma function.

Definition 6.24 (Standard embeddings of the Lagrangian catenoid).

(i) Suppose $n>2$. Let $w_{1}: \mathbb{R} \rightarrow \mathbb{C}^{*}$ be the unique solution of (6.22) with $w_{1}(0)=\mathrm{e}^{\mathrm{i} \pi / 2 n}$ as above and let $2 T$ denote the lifetime defined in (6.23). We call the special Lagrangian embedding $X_{1}:(-T, T) \times \mathbb{S}^{n-1} \rightarrow \mathbb{C}^{n}$ defined by

$$
X_{1}(t, \sigma)=w_{1}(t) \sigma, \quad \text { for } t \in(-T, T), \quad \sigma \in \mathbb{S}^{n-1} \subset \mathbb{R}^{n},
$$

the standard embedding of the Lagrangian catenoid of size 1 or just the standard unit Lagrangian catenoid for short.

(ii) Suppose $n=2$. Let $w_{1}: \mathbb{R} \rightarrow \mathbb{C}^{*}$ be the unique solution of (6.22) with $w_{1}(0)=\mathrm{e}^{\mathrm{i} \pi / 4}$, i.e.,

$$
w_{1}(t)=\frac{1}{\sqrt{2}}\left(e^{t}+i e^{-t}\right)
$$

We call the special Lagrangian embedding $X_{1}: \mathbb{R} \times \mathbb{S}^{1} \rightarrow \mathbb{C}^{2}$ defined by

$$
X_{1}(t, \sigma):=w_{1}(t) \sigma, \quad \text { for } t \in \mathbb{R}, \sigma \in \mathbb{S}^{1} \subset \mathbb{R}^{2},
$$

the standard embedding of the two-dimensional Lagrangian catenoid of size 1 or just the standard unit two-dimensional Lagrangian catenoid for short.

Remark 6.25. If we replace $w_{1}$ above with the rescaled solution of (6.22) given by

$$
w_{\lambda}(t):=\lambda^{1 / n} w_{1}\left(\lambda^{1-2 / n} t\right)
$$

and define $X_{\lambda}(t, \sigma)=w_{\lambda}(t) \sigma$ then we obtain the standard embedding of the Lagrangian catenoid of size $\lambda^{1 / n}$, i.e., the waist of $X_{\lambda}$ is a round sphere 
The geometry of $\mathrm{SO}(p) \times \mathrm{SO}(q)$-invariant special Lagrangian cones 231

of radius $\lambda^{1 / n}$. The solution $w_{\lambda}$ is defined on the interval $\lambda^{-1+2 / n}(-T, T)$, where $2 T$ is the lifetime of the solution $w_{1}$.

The limiting geometry of the necks for $\boldsymbol{p}=1$. Recall that for $p=1$ all waists are isometric and $\operatorname{Isom}\left(\mathrm{Cyl}^{p, q}, g_{\tau}\right)$ acts transitively on the waists. Hence without loss of generality we can concentrate on the neck containing the first waist $W[1]:=\left\{\mathrm{p}_{\tau}\right\} \times \mathbb{S}^{n-2}$. We first magnify the immersion $X_{\tau}$ while simultaneously repositioning and scaling the $t$ variable, by taking

$$
\widetilde{X}_{\tau}(\tilde{t}, \sigma)=\frac{1}{\beta} W X_{\tau}\left(\beta^{3-n} \tilde{t}+\mathbf{p}_{\tau}, \sigma\right),
$$

where $W \in \mathrm{U}(n)$ and $\beta>0$ are defined by

$$
\beta:=\left|w_{2}\left(\mathbf{p}_{\tau}\right)\right|=\sqrt{y_{\min }}, \quad W=\left(\begin{array}{cc}
\frac{\left|w_{1}\left(\mathbf{p}_{\tau}\right)\right|}{w_{1}\left(\mathbf{p}_{\tau}\right)} & 0 \\
0 & \mathrm{e}^{\mathrm{i} \pi / 2(n-1)} \frac{\left|w_{2}\left(\mathbf{p}_{\tau}\right)\right|}{w_{2}\left(\mathbf{p}_{\tau}\right)} \operatorname{Id}_{n-1}
\end{array}\right) .
$$

Geometrically, $\beta$ is the radius of the sphere $\mathbb{S}^{n-2}$ on the waist. Note that using (3.12) and that $\dot{y}\left(\mathbf{p}_{\tau}\right)=0$ we have $\operatorname{det} W=-1$. We can write (recall $(3.29))$

$$
\widetilde{X}_{\tau}(\tilde{t}, \sigma)=\left(z_{1}(\tilde{t})+\frac{\sqrt{1-\beta^{2}}}{\beta}, z_{2}(\tilde{t}) \cdot \sigma\right)
$$

where

$$
\begin{aligned}
& z_{1}(\tilde{t})=\frac{\sqrt{1-\beta^{2}}}{\beta}\left(\frac{w_{1}\left(\beta^{3-n} \tilde{t}+\mathbf{p}_{\tau}\right)}{w_{1}\left(\mathbf{p}_{\tau}\right)}-1\right), \\
& z_{2}(\tilde{t})=\mathrm{e}^{\mathbf{i} \pi / 2(n-1)} \frac{w_{2}\left(\beta^{3-n} \tilde{t}+\mathbf{p}_{\tau}\right)}{w_{2}\left(\mathbf{p}_{\tau}\right)} .
\end{aligned}
$$

In terms of the new coordinates $z_{1}, z_{2}$ and the rescaled time parameter $\tilde{t}$, (3.8) is equivalent to

$$
\begin{aligned}
& \frac{d z_{1}}{d \tilde{t}}=\beta \bar{z}_{2}^{n-1}, \\
& \frac{d z_{2}}{d \tilde{t}}=\left(\sqrt{1-\beta^{2}}+\beta \bar{z}_{1}\right) \bar{z}_{2}^{n-2}, \quad \text { with initial data } z_{1}(0)=0, z_{2}(0)=\mathrm{e}^{\mathrm{i} \pi / 2(n-1)} .
\end{aligned}
$$

By standard ODE theory (6.30) has a unique (real analytic) maximal solution for each $\beta \in \mathbb{R}$, which we denote by $\mathbf{z}_{\beta}=\left(z_{1, \beta}, z_{2, \beta}\right)$, and which depends 
analytically on $\beta \in(-1,1)$. When $\beta=0$ the system simplifies: we obtain $z_{1,0} \equiv 0$ and $z_{2,0}$ satisfies equation (6.22) with $n$ replaced by $n-1$ and with initial condition $z_{2,0}(0)=\mathrm{e}^{\mathrm{i} \pi / 2(n-1)}$. Hence (recall 6.24) $X(\tilde{t}, \sigma):=z_{2,0}(\tilde{t}) \sigma$ is the standard embedding of the unit Lagrangian catenoid in $\mathbb{C}^{n-1}$. By modifying $(6.28)$, we define a new $\mathrm{SO}(n-1)$-invariant embedding $\widehat{X}_{\tau}:(-T, T) \times$ $\mathbb{S}^{n-2} \rightarrow \mathbb{C}^{n}$ by

$$
\widehat{X}_{\tau}(\tilde{t}, \sigma)=\left(\frac{\sqrt{1-\beta^{2}}}{\beta}, z_{2,0}(\tilde{t}) \cdot \sigma\right),
$$

where $2 T$ is the lifetime of the standard embedding of the unit Lagrangian catenoid in $\mathbb{C}^{n-1}$; as discussed in 6.24 the lifetime $2 T$ is finite when $n-1>2$ and infinite when $n-1=2 . \widehat{X}_{\tau}$ is independent of $\tau$ except for the translation by $\sqrt{1-\beta^{2}} / \beta$ in the first factor and its image is the standard unit $n-1$ dimensional Lagrangian catenoid in $\{0\} \times \mathbb{C}^{n-1} \subset \mathbb{C} \times \mathbb{C}^{n-1}$ translated in the $x$ direction of the extra $\mathbb{C}$ factor. As $\tau \rightarrow 0$ the translation makes the Lagrangian catenoid drift to infinity.

If we take $b$ large if $n=3$ or $b<T$ but close to $T$ if $n>3$, and we restrict $|\tilde{t}| \leq b$, the image under $\widehat{X}_{\tau}$ is a truncated Lagrangian catenoid which exhausts the whole Lagrangian catenoid as $b \rightarrow T-(b \rightarrow \infty$ when $n=3)$. By the smooth dependence of $\mathbf{z}_{\beta}$ on $\beta$, we conclude that if $\tau$ is small enough depending on $b$, and $\beta$ is defined as in (6.27), we have

$$
\begin{gathered}
\left\|\mathbf{z}_{\beta}-\mathbf{z}_{0}: C^{k}([-b, b])\right\| \leq C(b, k) \beta, \\
\left\|\widetilde{X}_{\tau}-\widehat{X}_{\tau}: C^{k}\left([-b, b] \times \operatorname{Mer}^{1, n-1}\right)\right\| \leq C(b, k) \beta,
\end{gathered}
$$

where we can use the standard metric of the cylinder or alternatively the pullback of the Euclidean metric by $\widehat{X}_{\tau}$ to define the $C^{k}$ norm, and the constant $C(b, k)$ depends only on $b$ and $k$.

The limiting geometry of the necks when $p>1$. Recall that for $p>1$ waists (and hence necks) come in two types: type 1 waists where the radius of the first spherical factor $\mathbb{S}^{p-1}$ is minimal and type 2 waists where the radius of the second spherical factor $\mathbb{S}^{q-1}$ is minimal. We concentrate now on the case of a type 2 neck.

Since all type 2 waists are isometric and $\operatorname{Isom}\left(\mathrm{Cyl}^{p, q}, g_{\tau}\right)$ acts transitively on them we can without loss of generality deal with the waist $W[1]=\left\{\mathrm{p}_{\tau}^{+}\right\} \times \operatorname{Mer}^{p, q}$ (recall (6.3)). As in the case $p=1$ we magnify the immersion $X_{\tau}$ while repositioning and scaling the $t$ variable, by taking

$$
\tilde{X}_{\tau}\left(\tilde{t}, \sigma_{1}, \sigma_{2}\right)=\frac{1}{\beta} W X_{\tau}\left(\beta^{2-q} \tilde{t}+\mathrm{p}_{\tau}^{+}, \sigma_{1}, \sigma_{2}\right)
$$


The geometry of $\mathrm{SO}(p) \times \mathrm{SO}(q)$-invariant special Lagrangian cones 233

where $W \in \mathrm{U}(n)$ and $\beta>0$ are defined by

$$
\beta:=\left|w_{2}\left(\mathbf{p}_{\tau}^{+}\right)\right|=\sqrt{y_{\min }}, \quad W=\left(\begin{array}{cc}
\frac{\left|w_{1}\left(\mathbf{p}_{\tau}^{+}\right)\right|}{w_{1}\left(\mathbf{p}_{\tau}^{+}\right)} \operatorname{Id}_{p} & 0 \\
0 & \mathrm{e}^{\mathrm{i} \pi / 2 q} \frac{\left|w_{2}\left(\mathbf{p}_{\tau}^{+}\right)\right|}{w_{2}\left(\mathbf{p}_{\tau}^{+}\right)} \operatorname{Id}_{q}
\end{array}\right)
$$

Geometrically, $\beta$ is the radius of the second spherical factor $\mathbb{S}^{q-1}$ on the waist. As in the previous case using (3.12) and that $\dot{y}\left(\mathrm{p}_{\tau}^{+}\right)=0$ we have $\operatorname{det} W=-1$. We can write (recall $(3.29)$ )

$$
\tilde{X}_{\tau}\left(\tilde{t}, \sigma_{1}, \sigma_{2}\right)=\left(\left(z_{1}(\tilde{t})+\frac{\sqrt{1-\beta^{2}}}{\beta}\right) \cdot \sigma_{1}, z_{2}(\tilde{t}) \cdot \sigma_{2}\right),
$$

where

$$
\begin{aligned}
& z_{1}(\tilde{t})=\frac{\sqrt{1-\beta^{2}}}{\beta}\left(\frac{w_{1}\left(\beta^{2-q} \tilde{t}+\mathbf{p}_{\tau}^{+}\right)}{w_{1}\left(\mathbf{p}_{\tau}^{+}\right)}-1\right), \\
& z_{2}(\tilde{t})=\mathrm{e}^{\mathrm{i} \pi / 2 q} \frac{w_{2}\left(\beta^{2-q} \tilde{t}+\mathrm{p}_{\tau}^{+}\right)}{w_{2}\left(\mathbf{p}_{\tau}^{+}\right)}
\end{aligned}
$$

In terms of the new coordinates $z_{1}, z_{2}$ and the rescaled time parameter $\tilde{t}$, (3.8) is equivalent to

$$
\begin{aligned}
& \frac{d z_{1}}{d \tilde{t}}=\beta\left(\sqrt{1-\beta^{2}}+\beta \bar{z}_{1}\right)^{p-1} \bar{z}_{2}^{q}, \\
& \frac{d z_{2}}{d \tilde{t}}=\left(\sqrt{1-\beta^{2}}+\beta \bar{z}_{1}\right)^{p} \bar{z}_{2}^{q-1},
\end{aligned}
$$

As in the case $p=1$ (6.37) has a unique maximal solution $\mathbf{z}_{\beta}=\left(z_{1, \beta}, z_{2, \beta}\right)$, for any $\beta \in \mathbb{R}$, which depends analytically on $\beta \in(-1,1)$. When $\beta=0$ (6.37) again simplifies: $z_{1,0} \equiv 0$ and $z_{2,0}$ satisfies the equation for the standard embedding of the unit Lagrangian catenoid in $\mathbb{C}^{q}$. Therefore following (6.31), we define a new $\mathrm{SO}(p) \times \mathrm{SO}(q)$-invariant embedding $\widehat{X}_{\tau}:(-T, T) \times$ $\operatorname{Mer}^{p, q} \rightarrow \mathbb{C}^{p+q}$

$$
\widehat{X}_{\tau}\left(\tilde{t}, \sigma_{1}, \sigma_{2}\right)=\left(\frac{\sqrt{1-\beta^{2}}}{\beta} \cdot \sigma_{1}, z_{2,0}(\tilde{t}) \cdot \sigma_{2}\right)
$$

where $2 T$ denotes the lifetime of the standard embedding of the unit Lagrangian catenoid in $\mathbb{C}^{q}$ (which as we already discussed is finite if $q>2$ and infinite if $q=2$ ). The image of $\widehat{X}_{\tau}$ is the product of a $p-1$ sphere 
$r \cdot \mathbb{S}^{p-1}$ of large radius $r=\sqrt{1-\beta^{2}} / \beta \simeq \beta^{-1}$ with a unit $q$-dimensional standard Lagrangian catenoid. Arguing as before we conclude that if $\tau$ is small enough depending on $b$, and $\beta$ is defined as in (6.34), we have

$$
\begin{gathered}
\left\|\mathbf{z}_{\beta}-\mathbf{z}_{0}: C^{k}([-b, b])\right\| \leq C(b, k) \beta, \\
\left\|\widetilde{X}_{\tau}-\widehat{X}_{\tau}: C^{k}\left([-b, b] \times \operatorname{Mer}^{p, q}\right)\right\| \leq C(b, k) \beta,
\end{gathered}
$$

where we use the pullback of the Euclidean metric by $\widehat{X}_{\tau}$ to define the $C^{k}$ norm, the constant $C(b, k)$ depends only on $b$ and $k$, and $b<T$.

Finally the case of any type 1 neck is similar except that $\widehat{X}_{\tau}$ now becomes an $\mathrm{SO}(p) \times \mathrm{SO}(q)$ - invariant embedding of the product of the unit standard Lagrangian catenoid in $\mathbb{C}^{p}$ with a large round $q-1$ sphere in $\mathbb{C}^{q}$. We omit the details.

Synthesis. We combine the results from the previous two sections to describe qualitatively the geometry of $X_{\tau}$ for small $\tau$.

Case $p=1$. In this case each domain of periodicity of $g_{\tau}$ contains a single bulge $\hat{S}[k] \subset \mathrm{Cyl}^{p, q}$. Inside each bulge we fixed a compact subset $S[k] \subset \hat{S}[k]$ (depending on the choice of a sufficiently large real number $b$ ) which we called an almost spherical region of $X_{\tau}$. As $\tau \rightarrow 0$ the image of the $k$ th almost spherical region $S[k]$ under $X_{\tau}$ tends to the complement of a small tubular neighbourhood of the marked set $\mathbb{M}[k] \subset \mathbb{S}[k]$ inside the $k$ th approximating sphere $\mathbb{S}[k]=\widetilde{\mathrm{T}}_{2 k \widehat{\mathrm{p}}_{\tau}} \mathbb{S}[0] . S[k]$ connects to its neighbouring almost spherical regions $S[k-1]$ and $S[k+1]$ via two small transition regions which are localized near the marked set $\mathbb{M}[k]= \pm \widetilde{\mathrm{T}}_{2 k \widehat{\mathrm{p}}_{\tau}}\left(e_{1}\right)$ of $\mathbb{S}[k]$. The core of each transition region - the necks - are the vicinity of the two boundary waists $W[k]$ and $W[k+1]$ of $\hat{S}[k]$. As $\tau \rightarrow 0$ on each neck $X_{\tau}$ approaches an embedding of the $n-1$ dimensional Lagrangian catenoid of size $\sqrt{y_{\min }} \simeq(2 \tau)^{1 / n-1}$ located close to one of the two points $\pm \widetilde{\mathbf{T}}_{2 k \widehat{\mathrm{p}}_{\tau}}\left(e_{1}\right)$.

In the limit as $\tau \rightarrow 0$ almost spherical regions tend to (subsets) of the approximating spheres, while a transition region connecting neighbouring almost spherical regions tends to a point of intersection of the corresponding approximating spheres. It follows from (4.24) and Proposition 7.41 that as $\tau \rightarrow 0$ the rotational period of $X_{\tau}$ satisfies

$$
\widetilde{\mathrm{T}}_{2 \widehat{\mathrm{p}}_{\tau}} \rightarrow\left(\begin{array}{cc}
-1 & 0 \\
0 & \mathrm{e}^{-\mathrm{i} \pi /(n-1)} \mathrm{Id}_{n-1}
\end{array}\right) .
$$

Hence in the $\tau \rightarrow 0$ limit, the real $n$-planes in $\mathbb{C}^{n}$ associated to the almost spherical region $S[0]$ and the almost spherical region $S[1]$ are $\mathbb{R} \oplus \mathbb{R}^{n-1}$ and 


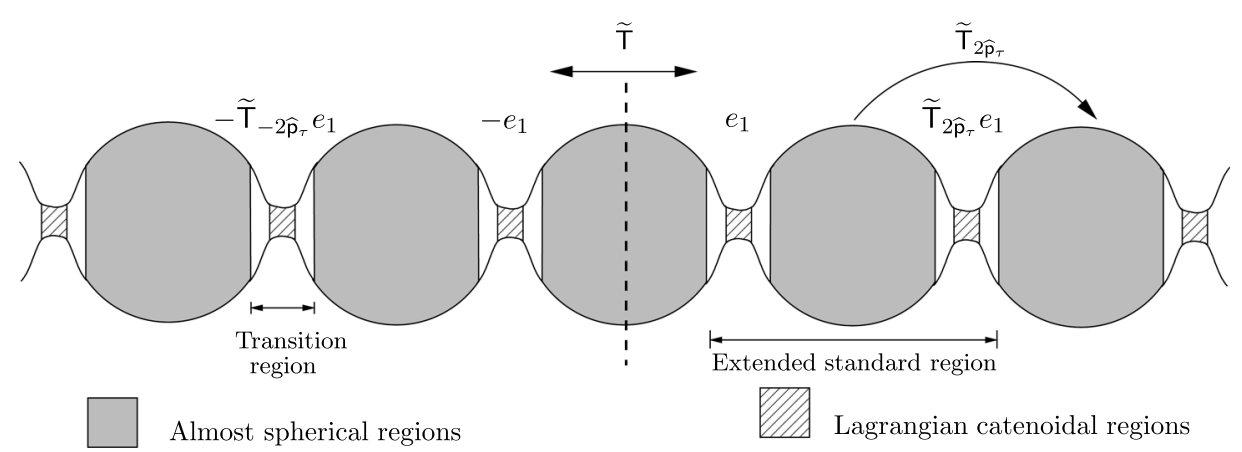

Figure 3: Schematic presentation of the intrinsic geometry of a special Legendrian cylinder $X_{\tau}$ with small $\tau$ and $p=1$.

$\mathbb{R} \oplus \mathrm{e}^{-\mathrm{i} \pi /(n-1)} \mathbb{R}^{n-1}$, respectively. This is consistent with the fact that the Lagrangian catenoid in $\mathbb{C}^{n-1}$ is asymptotic to the union of two $n-1$ planes (which up to rotation we can take to be) $\mathbb{R}^{n-1}$ and $\mathrm{e}^{-\mathrm{i} \pi /(n-1)} \mathbb{R}^{n-1}$. See figure 3 for a schematic illustration of the intrinsic geometry of $X_{\tau}$ in the case $p=1$.

Case $p>1$. In this case each domain of periodicity of $g_{\tau}$ contains not one but two bulges $\hat{S}[k]$ and $\hat{S}[k+1]$. For each bulge one of its two boundary waists is a waist of type 1 (where the radius of the first spherical factor $\mathbb{S}^{p-1}$ is minimal) and the other is a waist of type 2 (where the radius of the second spherical factor $\mathbb{S}^{q-1}$ is minimal). Moreover, since waists of types 1 and 2 alternate along $\mathrm{Cyl}^{p, q}$ one of the two bulges in a domain of periodicity will have a type 1 waist at its left-hand boundary and a type 2 waist at its right-hand boundary, whereas the other bulge will have a type 2 waist at its LH boundary and a type 1 waist at its RH boundary. Hence while the reflectional symmetry $\mathrm{T}_{2 k \mathrm{p}_{\tau}} \circ \mathrm{I}_{\mathrm{p}_{\tau}^{+}}$exchanges the adjacent almost spherical regions $S[k]$ and $S[k+1]$ there is no purely translational symmetry that achieves this (unlike the case $p=1$ ). Instead the basic rotational period $\widetilde{\mathrm{T}}_{2 \widehat{\mathrm{p}}_{\tau}}$ of $X_{\tau}$ sends $S[k]$ to $S[k+2]$. This reflects a fundamental difference in the geometry of $X_{\tau}$ between the cases $p=1$ and $p>1$.

Inside each bulge we fixed a compact subset $S[k] \subset \hat{S}[k]$ (depending on the choice of a sufficiently large real number $b$ ) which we called the $k$ th almost spherical region of $X_{\tau}$. As $\tau \rightarrow 0$ the image of the $k$ th almost spherical region $S[k]$ under $X_{\tau}$ tends to the complement of a small tubular neighbourhood of the marked set $\mathbb{M}[k] \subset \mathbb{S}[k]$ inside the $k$ th approximating sphere. The marked set $\mathbb{M}[k]$ is a generalized $(p, q)$-Hopf link, i.e., two orthogonal equatorial subspheres in $\mathbb{S}^{p+q-1}$ of dimensions $p-1$ and $q-1$. $S[k]$ connects to 
its neighbouring almost spherical regions $S[k-1]$ and $S[k+1]$ via two transition regions which are localized near the two components of the marked set $\mathbb{M}[k]$. The core of each transition region - the necks - is the vicinity of one of the waists $W[k]$ and $W[k+1]$ : one of type 1 and one of type 2 . On the neck containing the type 1 waist $X_{\tau}$ approaches an embedding of the product of a Lagrangian catenoid in $\mathbb{C}^{p}$ of size $\sqrt{1-y_{\max }} \simeq(2 \tau)^{1 / p}$ with a round sphere $\mathbb{S}^{q-1}$ of radius 1 as $\tau \rightarrow 0$. This type 1 neck localizes to the equatorial $q-1$ sphere of the marked set $\mathbb{M}[k]$. On the type 2 neck $X_{\tau}$ approaches an embedding of the product of a round sphere $\mathbb{S}^{p-1}$ of radius 1 with a Lagrangian catenoid in $\mathbb{C}^{q}$ of size $\sqrt{y_{\min }} \simeq(2 \tau)^{1 / q}$ which localizes to the equatorial $p-1$ sphere of the marked set $\mathbb{M}[k]$. In particular, when $p \neq q$ necks of type 1 and necks of type 2 are not isometric and hence no symmetry can take a type 1 neck to a type 2 neck.

However, when $p=q$ type 1 and type 2 necks are isometric and extra symmetries exist that exchange the two neck types; the symmetry $\underline{T}_{k p_{\tau}} \circ \mathrm{E}$ (recall 6.8 ) sends the bulge $\hat{S}[k]$ to itself but exchanges its two boundary waists $W[k]$ and $W[k+1]$.

In the limit as $\tau \rightarrow 0$ almost spherical regions tend to (subsets) of the approximating spheres, while a transition region connecting neighbouring almost spherical regions tends to the equatorial subsphere formed by the intersection of the corresponding approximate spheres. It follows from (4.24) and Proposition 7.41 that as $\tau \rightarrow 0$ the reflection $\widetilde{\underline{I}}_{+}$that sends $\mathbb{S}[0]$ to $\mathbb{S}[1]$ converges to the reflection

$$
(z, w) \mapsto\left(\bar{z}, \mathrm{e}^{-\mathrm{i} \pi / q} \bar{w}\right), \quad \text { for }(z, w) \in \mathbb{C}^{p} \times \mathbb{C}^{q} .
$$

Hence in the $\tau \rightarrow 0$ limit, the real $n$-planes in $\mathbb{C}^{n}$ associated to the almost spherical regions $S[0]$ and $S[1]$ are $\mathbb{R}^{p} \oplus \mathbb{R}^{q}$ and $\mathbb{R}^{p} \oplus \mathrm{e}^{-\mathrm{i} \pi / q} \mathbb{R}^{q}$. This is consistent both with the asymptotic geometry of the Lagrangian catenoid in $\mathbb{C}^{q}$ and the fact that the neck concentrates on a round $\mathbb{S}^{p-1}$. Similarly, the reflection $\widetilde{\mathbf{I}}_{-}$that sends $S[0]$ to $S[-1]$ converges as $\tau \rightarrow 0$ to the reflection

$$
(z, w) \mapsto\left(\mathrm{e}^{-\mathrm{i} \pi / p_{\bar{z}}}, \bar{w}\right), \quad \text { for }(z, w) \in \mathbb{C}^{p} \times \mathbb{C}^{q} .
$$

Hence in the $\tau \rightarrow 0$ limit the real $n$-planes in $\mathbb{C}^{n}$ associated to the almost spherical regions $S[0]$ and $S[-1]$ are $\mathbb{R}^{p} \oplus \mathbb{R}^{q}$ and $\mathrm{e}^{-\mathrm{i} \pi / p} \mathbb{R}^{p} \oplus \mathbb{R}^{q}$ respectively, which is again consistent with the asymptotic geometry of the $p$-dimensional catenoid and concentration of the neck on a round $\mathbb{S}^{q-1}$. 
The geometry of $\mathrm{SO}(p) \times \mathrm{SO}(q)$-invariant special Lagrangian cones 237

\section{Precise asymptotics as $\tau \rightarrow 0$}

In order to describe the asymptotics it helps to introduce the following notation: We define functions of $\tau$ by

$$
T_{k}(\tau):= \begin{cases}\tau^{-1+2 / k}, & \text { for } k>2 \\ \log \tau^{-1}, & \text { for } k=2,\end{cases}
$$

and introduce the notation $f_{1} \sim f_{2}$ for functions $f_{1}$ and $f_{2}$ of $\tau$ to mean that

$$
\frac{f_{2}(\tau)}{f_{1}(\tau)} \rightarrow 1 \quad \text { as } \quad \tau \rightarrow 0
$$

Using this notation we have the following:

\section{Proposition 7.3 (Small $\tau$ asymptotics of the period and partial- periods).}

(i) For $p>1, \mathrm{p}_{\tau}^{+}$and $\mathrm{p}_{\tau}^{-}$are analytic functions of $\tau$ for $0<|\tau|<\tau_{\max }$. For $p=1, \mathrm{p}_{\tau}$ is an analytic function of $\tau$ for $0<|\tau|<\tau_{\max }$.

(ii) In the case $p>1$ we have

$$
\mathrm{p}_{\tau}^{+} \sim b_{q} T_{q}(\tau), \quad \mathrm{p}_{\tau}^{-} \sim b_{p} T_{p}(\tau)
$$

where

$$
b_{2}:=\frac{1}{2}, \quad b_{k}:=4^{-1+\frac{1}{k}} \int_{1}^{\infty} \frac{d z}{\sqrt{z^{k}-1}}=4^{-1+\frac{1}{k}} \frac{\sqrt{\pi} \Gamma\left(\frac{1}{2}-\frac{1}{k}\right)}{\Gamma\left(-\frac{1}{k}\right)} \quad \text { for } k \geq 2,
$$

where $\Gamma$ is the gamma function. We also have

$$
\mathrm{p}_{\tau} \sim b_{q} T_{q}(\tau) \quad \text { when } \quad 1 \leq p<q, \quad \mathrm{p}_{\tau} \sim 2 b_{q} T_{q}(\tau) \quad \text { when } \quad 2 \leq p=q .
$$

Remark 7.7. For $k>2$, we note that the expression $b_{k} T_{k}(\tau)$ appearing in (7.4) is (recall 6.25) exactly half the lifetime of a Lagrangian catenoid in $\mathbb{C}^{k}$ of size $2 \tau$ parametrized by (6.22). In light of the geometry of the high curvature regions of $X_{\tau}$ described in Section 6 this does not come as a surprize. Note that $b_{2}=\frac{1}{2}$ in (7.5) corrects a misprint in [13, Prop 7.3].

Proof. See [13, Prop 7.3]. 
We introduce now some convenient notation. Note that the definition of $\Phi_{v}$ is motivated by the fact that if $\Phi$ is Legendrian then $\Phi_{v}$ is also Legendrian (see, e.g., [20, Lemma 2.4]).

Definition 7.8. If $\Phi: \Sigma \rightarrow \mathbb{S}^{2 n-1} \subset \mathbb{C}^{n}$ is an immersion and $V$ is a normal (to $\Phi$ in $\mathbb{S}^{2 n-1}$ ) small vector field, we define $\Phi_{V}: \Sigma \rightarrow \mathbb{S}^{2 n-1}$ by

$$
\Phi_{V}=\frac{\Phi+V}{|\Phi+V|},
$$

where we consider $\Phi$ and $V$ as $\mathbb{C}^{n}$-valued and $|$.$| is the standard length. If \Phi$ is Legendrian and $v: \Sigma \rightarrow \mathbb{R}$ a function with locally small enough $C^{1}$ norm, we also write $\Phi_{v}$ for $\Phi_{2 v J \frac{\partial}{\partial r}+J \nabla v}$.

In order to understand the asymptotics of $\widehat{\mathrm{p}}_{\tau}$ we prove first the following lemma. Recall the definition of the torque/flux in (3.32) and the calculation of the torque for $X_{\tau}$ given in Proposition 3.34.

Lemma 7.9. Let $\mathrm{t}=\left.\frac{d \widetilde{\mathrm{T}}_{x}}{d x}\right|_{x=0} \in \mathfrak{s u}(n)$ be the generator of the 1-parameter subgroup $\left\{\widetilde{\mathrm{T}}_{x}\right\}$. Suppose that $\left(X_{\tau}\right)_{\phi}$ is special Legendrian where $\phi: \mathrm{Cyl}^{p, q} \rightarrow$ $\mathbb{R}$ is a smooth function which depends only on $t$. The $\mathrm{t}$-flux through the meridian $\left(\{t\} \times \operatorname{Mer}^{p, q}\right)$ of $\left(X_{\tau}\right)_{\phi}$ is given by

$$
\mathcal{F}_{\mathrm{t}}=\frac{n}{p q} \operatorname{Vol}\left(\operatorname{Mer}^{p, q}\right)(2 \tau+(q-n y) \dot{\phi}+n \dot{y} \phi)+\text { h.o.t. }
$$

where $\operatorname{Vol}\left(\operatorname{Mer}^{p, q}\right)=\operatorname{Vol}\left(\mathbb{S}^{p-1}\right) \operatorname{Vol}\left(\mathbb{S}^{q-1}\right)$ if $p>1$ or $\operatorname{Vol}\left(\operatorname{Mer}^{p, q}\right)=\operatorname{Vol}\left(\mathbb{S}^{n-2}\right)$ if $p=1$, and "h.o.t." stands for terms which are quadratic or higher order in $\phi$ and its derivatives.

\section{Remark 7.10.}

(i) Since $\phi$ depends only on $t$ the linearized equation

$$
\Delta_{X^{*} g_{\mathbb{S}^{2 n-1}}} \phi+2 n \phi=0
$$

reduces to

$$
\ddot{\phi}=-2 n|\dot{\mathbf{w}}|^{2} \phi
$$

By (3.16) and (3.14) we have

$$
((q-n y) \dot{\phi}+n \dot{y} \phi)^{\cdot}=(q-n y)|\dot{\mathbf{w}}|^{2}\left(\Delta_{X^{*} g_{\mathbb{S}^{2 n-1}}} \phi+2 n \phi\right),
$$


The geometry of $\mathrm{SO}(p) \times \mathrm{SO}(q)$-invariant special Lagrangian cones 239

which shows that for any constant $A$ the first order linear ODE

$$
(q-n y) \dot{\phi}+n \dot{y} \phi=A
$$

is a first integral of the second order linearized Equation (7.11). Clearly, $\phi=q-n y$ satisfies (7.12) with $A=0$ and hence is a solution of the linearized Equation (7.11).

(ii) There is a simple geometric explanation for the solution $q-n y$ to the rotationally invariant linearized equation including for the fact that it satisfies (7.12) with constant $A=0$; to explain this recall the discussion in 3.6. For any special Legendrian $X$ the variation vector field $V$ associated with the 1-parameter variation $\widetilde{\mathrm{T}}_{x} \circ X$ arises from a function $\varphi$ which solves the linearized equation. The function $\varphi$ is $f_{\mathrm{t}} \circ X$, where

$$
f_{\mathrm{t}}\left(z_{1}, \ldots, z_{n}\right)=\frac{1}{2 p} \sum_{i=1}^{p}\left|z_{i}\right|^{2}-\frac{1}{2 q} \sum_{i=p+1}^{n}\left|z_{i}\right|^{2},
$$

which satisfies

$$
J \nabla f_{\mathrm{t}}=\mathrm{t}=\left.\frac{d \widetilde{\mathbf{T}}_{x}}{d x}\right|_{x=0},
$$

i.e., it is the function whose associated Hamiltonian vector field is $\mathrm{t}$, the infinitesimal generator of $\left\{\widetilde{\mathrm{T}}_{x}\right\}$. Recall that the 1-parameter subgroup $\left\{\widetilde{\mathrm{T}}_{x}\right\}$ commutes with every $\mathrm{O} \in \mathrm{SO}(p) \times \mathrm{SO}(q)$. Hence for any $x$, $\widetilde{\mathrm{T}}_{x} \circ X_{\tau}$ is also an $\mathrm{SO}(p) \times \mathrm{SO}(q)$-invariant special Legendrian congruent to $X_{\tau}$. Since $f_{\mathrm{t}}$ is $\mathrm{SO}(p) \times \mathrm{SO}(q)$-invariant its restriction to every meridian is constant, i.e., $f_{\mathrm{t}} \circ X_{\tau}$ is some rotationally invariant function. Calculation shows this function is $f_{\mathrm{t}} \circ X_{\tau}=\frac{q-n y}{2 p q} \cdot q-n y$ satisfies (7.12) with $A=0$ because $\widetilde{\mathrm{T}}_{x} \circ X_{\tau}$ is just a repositioning of $X_{\tau}$.

(iii) The variation vector field corresponding to varying $\tau$ in $X_{\tau}$ also arises from a rotationally invariant solution $\varphi$ of the linearized equation, but by Lemma 7.9 it must satisfy (7.12) with a nonzero constant $A$.

(iv) Recall (see, e.g., [1, Section 27]) that if $\psi$ and $\phi$ are solutions of the second order linear equation (7.11) then the Wronskian $W_{\psi, \phi}(t):=$ $\psi \dot{\phi}-\dot{\psi} \phi$ is constant and $\psi, \phi$ span the space of solutions of (7.11) if and only if this constant is nonzero. The expression on the LHS of (7.12) is precisely the Wronskian where $\psi=q-n y$. Hence to find 
a basis of solutions for (7.11) it suffices to find a function $\phi$ satisfying (7.12) with say $A=1$.

Proof. The proof is a rather long calculation making full use of the expression for $X_{\tau}$ in terms of $\mathbf{w}_{\tau}$, the definition of the perturbation of a Legendrian submanifold by a function and repeated use of the equations satisfied by $\mathbf{w}_{\tau}$, in particular (3.8), (3.12), (3.13) and (3.16). Because of the importance of the lemma for calculating the asymptotics of $\widehat{p}_{\tau}$ as $\tau \rightarrow 0$ we outline some important steps in the calculation.

To simplify the notation we write $X=X_{\tau}$ and $Y=\left(X_{\tau}\right)_{\phi}$. To compute the t-flux through the meridian $\left(\{t\} \times \operatorname{Mer}^{p, q}\right)$ of $Y$ using (3.32) we need to compute the following: the pullback metric $Y^{*} g_{\mathbb{S}^{2 n-1}}$, the unit conormal $\eta$ to the meridian, the volume form induced on the meridian by $Y^{*} g_{\mathbb{S}^{2 n-1}}$ and the inner product $\eta \cdot \mathrm{t}$.

We proceed to calculate $Y^{*} g_{\mathbb{S}^{2 n-1}}$. We first assume that $p>1$. The definition of $(X)_{\phi}$ yields

$$
Y=X+i|\dot{\mathbf{w}}|^{-2} \dot{\phi} \dot{X}+2 i \phi X+\text { h.o.t. }
$$

and therefore

$$
\dot{Y}=\dot{X}+i \ddot{X}\left(|\dot{\mathbf{w}}|^{-2} \dot{\phi}\right)+i \dot{X}\left(\dot{\phi}\left(|\dot{\mathbf{w}}|^{-2}\right)^{\cdot}+|\dot{\mathbf{w}}|^{-2} \ddot{\phi}+2 \phi\right)+2 i X \dot{\phi}+\text { h.o.t. }
$$

We compute that

$$
|\dot{Y}|^{2}=|\dot{X}|^{2}+2|\dot{\mathbf{w}}|^{-2} \dot{\phi} \dot{X} \cdot i \ddot{X}+\text { h.o.t. }
$$

(many terms vanish using the fact that $X$ is Legendrian in $\mathbb{S}^{2 n-1}$ and because we only keep terms linear in $\phi$ and its derivatives). From the definition of $X_{\tau}$ in terms of $\mathbf{w}_{\tau}$ we find

$$
\dot{X} \cdot i \ddot{X}=\operatorname{Im}\left(\dot{w}_{1} \ddot{\bar{w}}_{1}+\dot{w}_{2} \ddot{\bar{w}}_{2}\right)
$$

Differentiation of (3.8) to compute the second derivatives of $\mathbf{w}$ and subsequent persistent use of (3.8) to replace all first derivatives of $\mathbf{w}$ eventually yields

$$
\begin{aligned}
\dot{w}_{1} \ddot{\bar{w}}_{1}+\dot{w}_{2} \ddot{\bar{w}}_{2}= & \bar{w}_{1}^{p} \bar{w}_{2}^{q}(1-y)^{p-1} y^{q-1} \\
& \times\left((p-1) \frac{\left|w_{2}\right|^{2}}{\left|w_{1}\right|^{2}}+(p-q)-(q-1) \frac{\left|w_{1}\right|^{2}}{\left|w_{2}\right|^{2}}\right)
\end{aligned}
$$


The geometry of $\mathrm{SO}(p) \times \mathrm{SO}(q)$-invariant special Lagrangian cones 241

Using (3.16), (3.12) and some algebraic manipulation we obtain $\dot{X} \cdot i \ddot{X}=$ $2 \tau|\dot{\mathbf{w}}|^{2}\left(\frac{p-1}{\left|w_{1}\right|^{2}}-\frac{q-1}{\left|w_{2}\right|^{2}}\right)$, and hence

$$
|\dot{Y}|^{2}=|\dot{\mathbf{w}}|^{2}+4 \tau\left(\frac{p-1}{\left|w_{1}\right|^{2}}-\frac{q-1}{\left|w_{2}\right|^{2}}\right) \dot{\phi}+\text { h.o.t. }
$$

A straightforward but somewhat lengthy calculation shows that for $p>1$

$$
\begin{aligned}
Y^{*} g_{\mathbb{S}^{2 n-1}}= & \left(|\dot{\mathbf{w}}|^{2}+4 \tau\left(\frac{p-1}{\left|w_{1}\right|^{2}}-\frac{q-1}{\left|w_{2}\right|^{2}}\right) \dot{\phi}\right) d t^{2} \\
& +\left(\left|w_{1}\right|^{2}-4 \tau|\dot{\mathbf{w}}|^{-2} \dot{\phi}\right) g_{\mathbb{S}^{p-1}}+\left(\left|w_{2}\right|^{2}+4 \tau|\dot{\mathbf{w}}|^{-2} \dot{\phi}\right) g_{\mathbb{S}^{q-1}}+\text { h.o.t. }
\end{aligned}
$$

and for $p=1$

$$
\begin{aligned}
Y^{*} g_{\mathbb{S}^{2 n-1}}= & \left(|\dot{\mathbf{w}}|^{2}-4 \tau(n-2)\left|w_{2}\right|^{-2} \dot{\phi}\right) d t^{2} \\
& +\left(\left|w_{2}\right|^{2}+4 \tau|\dot{\mathbf{w}}|^{-2} \dot{\phi}\right) g_{\mathbb{S}^{n-2}}+\text { h.o.t. }
\end{aligned}
$$

When $\phi=0$ these expressions reduce to the induced metric $g_{\tau}$ given in 3.30.ii. Hence in both cases the unit conormal $\eta$ to the meridian $\{t\} \times \mathrm{Mer}^{p, q}$ is $\eta=\frac{\dot{Y}}{|\dot{Y}|}+$ h.o.t. Combining this with (7.18) and (7.19), we have

$$
\eta \cdot \mathrm{t} d \mathrm{v}= \begin{cases}\left(1-4 \tau|\dot{\mathrm{w}}|^{-2}\left(\frac{p-1}{\left|w_{1}\right|^{2}}-\frac{q-1}{\left|w_{2}\right|^{2}}\right) \dot{\phi}\right) \dot{Y} \cdot \mathrm{t} d \mathrm{v}_{\mathbb{S}^{p-1}} d \mathrm{v}_{\mathbb{S} q-1}+\text { h.o.t. } & \text { if } p>1 \\ \left(1+4 \tau(n-2)|\dot{\mathbf{w}}|^{-2}\left|w_{2}\right|^{-2} \dot{\phi}\right) \dot{Y} \cdot \mathrm{t} d \mathrm{v}_{\mathbb{S}^{n-2}}+\text { h.o.t. } & \text { if } p=1 .\end{cases}
$$

It remains to calculate $\dot{Y} \cdot \mathrm{t}$. Recall that $\mathrm{t}$ at the point $\left(z_{1}, \ldots, z_{n}\right) \in \mathbb{C}^{n}$ is given by

$$
\mathrm{t}\left(z_{1}, \ldots, z_{n}\right)=i\left(z_{1} / p, \ldots, z_{p} / p,-z_{p+1} / q, \ldots,-z_{n} / q\right) .
$$

Since $Y$ is special Legendrian, and $\phi$ depends only on $t$ it satisfies the linearized equation (7.11). Substituting (7.11) into (7.16) and also using the 
expression for $Y$ from (7.15) we calculate that

$$
\begin{aligned}
\dot{Y} \cdot \mathrm{t}=\frac{1}{p} \operatorname{Re}( & -i \dot{w}_{1} \bar{w}_{1}+\ddot{w}_{1} \bar{w}_{1}|\dot{\mathbf{w}}|^{-2} \dot{\phi}+\dot{w}_{1} \bar{w}_{1}\left(|\dot{\mathbf{w}}|^{-2}\right)^{\cdot} \dot{\phi} \\
& \left.-\dot{w}_{1} \bar{w}_{1} 2 n \phi+\left|w_{1}\right|^{2} 2 \dot{\phi}-\left|\dot{w}_{1}\right|^{2}|\dot{\mathbf{w}}|^{-2} \dot{\phi}\right) \\
-\frac{1}{q} \operatorname{Re} & \left(-i \dot{w}_{2} \bar{w}_{2}+\ddot{w}_{2} \bar{w}_{2}|\dot{\mathbf{w}}|^{-2} \dot{\phi}+\dot{w}_{2} \bar{w}_{2}\left(|\dot{\mathbf{w}}|^{-2}\right)^{\cdot} \dot{\phi}\right. \\
& \left.-\dot{w}_{2} \bar{w}_{2} 2 n \phi+\left|w_{2}\right|^{2} 2 \dot{\phi}-\left|\dot{w}_{2}\right|^{2}|\dot{\mathbf{w}}|^{-2} \dot{\phi}\right) .
\end{aligned}
$$

We claim that this expression for $\dot{Y} \cdot \mathrm{t}$ can be simplified to

$$
\dot{Y} \cdot \mathbf{t}=\frac{n}{p q}\left(2 \tau+\left(q\left|w_{1}\right|^{2}-p\left|w_{2}\right|^{2}+8 \tau^{2}|\dot{\mathbf{w}}|^{-2}\left(\frac{p-1}{\left|w_{1}\right|^{2}}-\frac{q-1}{\left|w_{2}\right|^{2}}\right)\right) \dot{\phi}+n \dot{y} \phi\right) .
$$

Granted this claim the Lemma follows by using (7.20) and (7.21) to evaluate the t-flux integral (3.32) up to higher order terms. For completeness, we indicate how to obtain (7.21). The zero order terms and the terms involving only $\phi$ are easily computed using (3.8), (3.12) and (3.16). Combining the eight terms involving $\dot{\phi}$ in the expression above (7.21) to yield the coefficient of $\dot{\phi}$ in (7.21) is more involved. First, we rewrite the eight terms appearing as the coefficient of $\dot{\phi}$ in the form

$$
\begin{aligned}
\frac{1}{p}( & \left.\partial_{t}\left(\operatorname{Re}\left(\bar{w}_{1} \dot{w}_{1}\right)|\dot{\mathbf{w}}|^{-2}\right)+2\left|w_{1}\right|^{2}-2\left|w_{2}^{2}\right|\right) \\
& -\frac{1}{q}\left(\partial_{t}\left(\operatorname{Re}\left(\bar{w}_{2} \dot{w}_{2}\right)|\dot{\mathbf{w}}|^{-2}\right)+2\left|w_{2}\right|^{2}-2\left|w_{1}^{2}\right|\right) \\
= & \frac{n}{p q}\left(\partial_{t}\left(\operatorname{Re}\left(\bar{w}_{1} \dot{w}_{1}\right)|\dot{\mathbf{w}}|^{-2}\right)+2\left|w_{1}\right|^{2}-2\left|w_{2}\right|^{2}\right) .
\end{aligned}
$$

Rewrite $\operatorname{Re}\left(\bar{w}_{1} \dot{w}_{1}\right)|\dot{\mathbf{w}}|^{-2}$ as $\operatorname{Re}\left(\bar{w}_{1} \bar{w}_{2} w_{1}^{1-p} w_{2}^{1-q}\right)$ using (3.8) and (3.16). Repeated use of (3.8) yields

$$
\partial_{t}\left(\bar{w}_{1} \bar{w}_{2} w_{1}^{1-p} w_{2}^{1-q}\right)=\left|w_{2}\right|^{2}-\left|w_{1}\right|^{2}+\frac{\bar{w}_{1}^{p} \bar{w}_{2}^{q}}{w_{1}^{p} w_{2}^{q}}\left((1-p)\left|w_{2}\right|^{2}-(1-q)\left|w_{1}\right|^{2}\right),
$$

while (3.16), (3.12) and (3.13) imply that

$$
\operatorname{Re}\left(\frac{\bar{w}_{1}^{p} \bar{w}_{2}^{q}}{w_{1}^{p} w_{2}^{q}}\right)=1-\frac{8 \tau^{2}}{f(y)}=1-\frac{8 \tau^{2}}{|\dot{\mathbf{w}}|^{2}\left|w_{1}\right|^{2}\left|w_{2}\right|^{2}} .
$$


The geometry of $\mathrm{SO}(p) \times \mathrm{SO}(q)$-invariant special Lagrangian cones 243

Hence

$$
\begin{aligned}
\partial_{t}\left(\operatorname{Re}\left(\bar{w}_{1} \dot{w}_{1}\right)|\dot{\mathbf{w}}|^{-2}\right)= & (2-p)\left|w_{2}\right|^{2}-(2-q)\left|w_{1}\right|^{2} \\
& +8 \tau^{2}|\dot{\mathbf{w}}|^{-2}\left(\frac{p-1}{\left|w_{1}\right|^{2}}-\frac{q-1}{w_{2}^{2}}\right) .
\end{aligned}
$$

Combining (7.22) with (7.23) gives us the coefficient of $\dot{\phi}$ as it appears in (7.21).

The asymptotics of the angular period $\widehat{p}_{\tau}$ as $\tau \rightarrow 0$ will follow from the following result which expresses the derivative of the angular period for all values of $\tau$ in terms of the behaviour of a particular (rotationally invariant) solution $Q$ (depending on $\tau$ ) of the linearized equation (7.11).

Lemma 7.24. The angular period $\widehat{\mathrm{p}}_{\tau}$ is an analytic function of $\tau$ for $\tau \in\left(0, \tau_{\max }\right)$. For any $0<\tau<\tau_{\max }$ the derivative of the angular period $\widehat{\mathrm{p}}_{\tau}$ satisfies

$$
\frac{d \widehat{\mathbf{p}}_{\tau}}{d \tau}=4(n-1)\left(\frac{Q\left(\mathrm{p}_{\tau}\right)}{q-n y\left(\mathrm{p}_{\tau}\right)}-\frac{Q(0)}{q-n y(0)}\right), \quad \text { when } p=1
$$

$$
\frac{d \widehat{\mathbf{p}}_{\tau}}{d \tau}=4 p q\left(\frac{Q\left(\mathrm{p}_{\tau}^{+}\right)}{q-n y\left(\mathrm{p}_{\tau}^{+}\right)}-\frac{Q\left(-\mathrm{p}_{\tau}^{-}\right)}{q-n y\left(-\mathrm{p}_{\tau}^{-}\right)}\right), \quad \text { when } p>1
$$

where $Q(t)$ is the unique solution to the rotationally-invariant linearized equation (7.11) with initial data

$$
\begin{aligned}
n \dot{y}\left(\mathrm{p}_{\tau}^{*}\right) Q\left(\mathrm{p}_{\tau}^{*}\right)=1, & \dot{Q}\left(\mathrm{p}_{\tau}^{*}\right)=0, \quad \text { when } p=1 \\
n \dot{y}(0) Q(0)=1, & \dot{Q}(0)=0, \quad \text { when } p>1
\end{aligned}
$$

where for $p=1 \mathrm{p}_{\tau}^{*}$ is the unique $t \in\left(0, \mathrm{p}_{\tau}\right)$ such that $y\left(\mathrm{p}_{\tau}^{*}\right)=\frac{n-1}{n}=\frac{q}{n}$.

Remark 7.29. For $p=1 y\left(\mathbf{p}_{\tau}^{*}\right)=q / n$ and $\mathrm{p}_{\tau}^{*}$ is locally characterized by this property. Also $\dot{y}\left(\mathrm{p}_{\tau}^{*}\right)=-4 \sqrt{\tau_{\max }^{2}-\tau^{2}} \neq 0$ for $\tau \in\left(-\tau_{\max }, \tau_{\max }\right)$. Hence by the real analytic Implicit Function Theorem $\mathrm{p}_{\tau}^{*}$ is an analytic function of $\tau$ in $\left(-\tau_{\max }, \tau_{\max }\right)$. In particular, it is bounded independent of $\tau$ as $\tau \rightarrow 0$. For $p>1 y(0)=q / n$ and $\dot{y}(0)=-4 \sqrt{\tau_{\max }^{2}-\tau^{2}}$.

Hence in both cases the initial conditions for $Q$ vary analytically with $|\tau|<\tau_{\max }$. Also by 3.30.i the coefficients of the linearized equation (7.11) depend analytically on $\tau \in\left(-\tau_{\max }, \tau_{\max }\right)$. Combining all these facts we see that the solution $Q$ to (7.11) defined above depends analytically on $\tau \in$ 
$\left(-\tau_{\max }, \tau_{\max }\right)$. Therefore, if $t$ stays in a bounded interval $I \subset \mathbb{R}$ then $\sup _{t \in I}|Q(t)|$ is bounded independent of $\tau$ as $\tau \rightarrow 0$. In particular, the term $Q(0)$ appearing in (7.25) is bounded as $\tau \rightarrow 0$.

Proof. Real analyticity of $\widehat{\mathbf{p}}_{\tau}$ for $\tau \in\left(0, \tau_{\max }\right)$ follows from real analyticity of $\mathbf{w}_{\tau}, \mathrm{p}_{\tau}^{+}, \mathrm{p}_{\tau}^{-}$and $\mathrm{p}_{\tau}$ and the definition of $\widehat{\mathrm{p}}_{\tau}(4.23)$. We fix any $\tau \in\left(0, \tau_{\max }\right)$ and consider $\sigma$ sufficiently close to $\tau$ which we will allow to vary.

Consider first the case $p=1$. By $5.15 X:=X_{\tau}$ has the symmetries

$$
\underline{\mathbf{T}} \circ X=X \circ \underline{\mathrm{T}}, \quad \underline{\widetilde{\mathrm{T}}}_{\widehat{\mathrm{p}}_{\tau}} \circ X=X \circ \underline{\mathrm{T}}_{\mathrm{p}_{\tau}} .
$$

$Y:=X_{\sigma}$ shares the $\underline{\tilde{I}}$ symmetry

$$
\underline{\mathbf{T}} \circ Y=Y \circ \underline{\mathrm{T}},
$$

but not the symmetry with respect to $\underline{\widetilde{T}}_{\widehat{\mathrm{p}}_{\tau}}$ (because we have changed from $\tau$ to $\sigma$ ). However, the following repositioned and reparametrized version of $X_{\sigma}$

$$
Z:=\widetilde{\mathrm{T}}_{\widehat{\mathbf{p}}_{\tau}-\widehat{\mathrm{p}}_{\sigma}} \circ X_{\sigma} \circ \mathrm{T}_{\mathbf{p}_{\sigma}-\mathrm{p}_{\tau}}
$$

does share the other ( $\sigma$-independent) symmetry of $X$, i.e.,

$$
\underline{\underline{\mathrm{T}}}_{\widehat{\mathrm{p}}_{\tau}} \circ Z=Z \circ \underline{\mathrm{T}}_{\mathrm{p}_{\tau}} \text {. }
$$

Since $\left\{\widetilde{\mathrm{T}}_{x}\right\}$ commutes with $\mathrm{O}(n-1)$ by 5.20 .ii the immersion $Z$ is $\mathrm{O}(n-1)$ invariant like $X$ and $Y$.

When $p>1$ we write

$$
X:=X_{\tau}, \quad Y:=\widetilde{\mathrm{T}}_{x^{-}} \circ X_{\sigma} \circ \mathrm{T}_{\mathrm{p}_{\tau}^{-}-\mathrm{p}_{\sigma}^{-}}, \quad Z:=\widetilde{\mathrm{T}}_{\widehat{\mathrm{p}}_{\tau}-\widehat{\mathrm{p}}_{\sigma}} \circ Y \circ \mathrm{T}_{\mathrm{p}_{\sigma}-\mathrm{p}_{\tau}},
$$

where $x^{-}$is defined to be the small number which ensures that the symmetries of $X$ in (5.23d) and (5.23c) (or (5.29d) and (5.29c)) apply to $Y$ and $Z$ respectively as

$$
\underline{\underline{T}}_{-} \circ Y=Y \circ \underline{\mathrm{T}}_{-\mathrm{p}_{\tau}^{-}}
$$

and

$$
\underline{\underline{\mathbf{T}}}_{+} \circ Z=Z \circ \underline{\mathrm{T}}_{\mathrm{p}_{\tau}^{+}},
$$

where $\underline{\mathbf{T}}_{-}$and $\underline{\mathbf{T}}_{+}$are defined in $(5.24 \mathrm{~b})$ and $(5.24 \mathrm{a})$ respectively (and are independent of $\sigma$ ). 
The geometry of $\mathrm{SO}(p) \times \mathrm{SO}(q)$-invariant special Lagrangian cones 245

Provided $\sigma$ is sufficiently close to $\tau$ we clearly have unique small vector fields $V$ and $W$ normal to $\left.X\right|_{\left(-2 \mathrm{p}_{\tau}, 2 \mathrm{p}_{\tau}\right) \times \operatorname{Mer}^{p, q}}$, and diffeomorphisms close to the identity $D_{\sigma}, E_{\sigma}:\left(-2 \mathrm{p}_{\tau}, 2 \mathrm{p}_{\tau}\right) \times \mathrm{Mer}^{p, q} \rightarrow \mathrm{Cyl}^{p, q}$, such that on $\left(-2 \mathrm{p}_{\tau}, 2 \mathrm{p}_{\tau}\right) \times \operatorname{Mer}^{p, q}$

$$
Y=X_{V} \circ D_{\sigma}, \quad \text { and } \quad Z=X_{W} \circ E_{\sigma} .
$$

Clearly $V, W, D_{\sigma}, E_{\sigma}$ are smooth and depend smoothly on $\sigma$. Moreover by the appropriate version of the Legendrian neighbourhood theorem (see, e.g., [20, Lemma 2.4]) there are unique small smooth functions $\widetilde{\phi}_{\sigma}, \widetilde{\varphi}_{\sigma}:\left(-2 \mathrm{p}_{\tau}, 2 \mathrm{p}_{\tau}\right) \times$ $\mathrm{Mer}^{p, q} \rightarrow \mathbb{R}$ depending smoothly on $\sigma$ such that

$$
V=2 \widetilde{\phi}_{\sigma} J \frac{\partial}{\partial r}+J \nabla \widetilde{\phi}_{\sigma}, \quad W=2 \widetilde{\varphi}_{\sigma} J \frac{\partial}{\partial r}+J \nabla \widetilde{\varphi}_{\sigma},
$$

and therefore by $7.8 X_{V}=\underset{\widetilde{\phi}_{\sigma}}{X_{\widetilde{\phi}_{\sigma}}}$ and $X_{W}=X_{\widetilde{\varphi}_{\sigma}}$.

We want to show that $\widetilde{\phi}_{\sigma}$ and $\widetilde{\varphi}_{\sigma}$ inherit certain symmetries from the symmetries of $X, Y$ and $Z$ given above. We claim that $\widetilde{\phi}_{\sigma}$ and $\widetilde{\varphi}_{\sigma}$ depend only on $t$ and that

$$
\begin{array}{rll}
-\widetilde{\phi}_{\sigma} \circ \underline{I}=\widetilde{\phi}_{\sigma}, & -\widetilde{\varphi}_{\sigma} \circ \underline{T}_{\mathbf{p}_{\tau}}=\widetilde{\varphi}_{\sigma} & \text { if } p=1 ; \\
-\widetilde{\phi}_{\sigma} \circ \underline{I}_{-\mathbf{p}_{\tau}^{-}}=\widetilde{\phi}_{\sigma}, & -\widetilde{\varphi}_{\sigma} \circ \underline{\mathbf{p}}_{\tau}^{+}=\widetilde{\varphi}_{\sigma} & \text { if } p>1 .
\end{array}
$$

To see the first symmetry of (7.34) we combine 5.20.iii with the $\widetilde{\underline{I}}$ symmetry of $X$ to obtain

$$
\widetilde{\mathbf{I}} \circ X_{\widetilde{\phi}_{\sigma}}=(\tilde{\underline{I}} \circ X)_{-\widetilde{\phi}_{\sigma}}=(X \circ \underline{\underline{T}})_{-\widetilde{\phi}_{\sigma}}=X_{-\widetilde{\phi}_{\sigma} \circ \underline{I}} \circ \underline{\underline{I}} .
$$

Combining this with the symmetry (7.30) of $Y$ we conclude

$$
X_{-\widetilde{\phi}_{\sigma} \circ \underline{\underline{I}}} \circ \underline{\mathrm{T}} \circ D_{\sigma}=X_{\widetilde{\phi}_{\sigma}} \circ D_{\sigma} \circ \underline{\mathrm{T}} .
$$

The uniqueness statement in the Legendrian neighbourhood theorem now implies that $-\widetilde{\phi}_{\sigma} \circ \underline{I}=\widetilde{\phi}_{\sigma}$ as required. Arguing in the same way using the symmetry of $X$ and $\bar{Z}$ (7.31) with respect to $\widetilde{\mathrm{I}}_{\widehat{\mathrm{p}}_{\tau}}$ we conclude that $\widetilde{\varphi}$ satisfies the second symmetry of (7.34). The analogous argument using the symmetry of $X, Y$ and $Z$ under any $\mathrm{O} \in \mathrm{O}(n-1)$ (recall (5.16a)) implies that

$$
\widetilde{\phi}_{\sigma} \circ \mathrm{O}=\widetilde{\phi}_{\sigma}, \quad \widetilde{\varphi}_{\sigma} \circ \mathrm{O}=\widetilde{\varphi}_{\sigma}, \quad \text { for any } \mathrm{O} \in \mathrm{O}(n-1),
$$

and therefore $\widetilde{\phi}_{\sigma}$ and $\widetilde{\varphi}_{\sigma}$ depend only on $t$. For $p>1$ the same sort of arguments establish the symmetries in (7.35) and the rotational symmetry of $\widetilde{\phi}_{\sigma}$ and $\widetilde{\varphi}_{\sigma}$. 
Linearizing $Z:=\widetilde{\mathrm{T}}_{\widehat{\mathrm{p}}_{\tau}-\widehat{\mathrm{p}}_{\sigma}} \circ Y \circ \mathrm{T}_{\mathbf{p}_{\sigma}-\mathrm{p}_{\tau}}$ around $\sigma=\tau$, using 7.14 and comparing normal components we obtain the following important equality

$$
\varphi=\phi-\left(\left.\frac{d \widehat{\mathrm{p}}_{\tau}}{d \tau}\right|_{\tau}\right) f_{\mathrm{t}} \circ X
$$

with $f_{\mathrm{t}}$ as defined in 7.13 and

$$
\varphi=\left.\frac{d \widetilde{\varphi}_{\sigma}}{d \sigma}\right|_{\sigma=\tau} \quad \text { and } \quad \phi=\left.\frac{d \widetilde{\phi}_{\sigma}}{d \sigma}\right|_{\sigma=\tau}
$$

Recall that

$$
f_{\mathrm{t}} \circ X=\frac{q-n y}{2 p q} .
$$

Differentiating the expression for the linearized t-flux from Lemma 7.9, we find that $\phi$ satisfies

$$
(q-n y) \dot{\phi}+n \dot{y} \phi=2 .
$$

Using the initial conditions for $Q$ given in (7.27) and (7.28) we see that the Wronskian $W(t)$ of $q-n y$ with $Q$ satisfies

$$
W(t):=(q-n y) \dot{Q}+n \dot{y} Q \equiv 1,
$$

and hence by Remark $7.10 Q$ and $q-n y$ span the solution space of the linearized equation (7.11). Hence from (7.37) there is a unique constant $b$ such that

$$
\phi=2(b(q-n y)+Q) .
$$

$\phi$ and $\varphi$ inherit the symmetries of $\widetilde{\phi}_{\sigma}$ and $\widetilde{\varphi}_{\sigma}$ (7.34) and (7.35) (for the case $p=1$ and $p>1$, respectively). In particular, we have

$$
\phi(0)=0, \quad \text { and } \quad \varphi\left(\mathbf{p}_{\tau}\right)=0 \quad \text { when } p=1 ;
$$

and

$$
\phi\left(-\mathrm{p}_{\tau}^{-}\right)=0, \quad \text { and } \quad \varphi\left(\mathrm{p}_{\tau}^{+}\right)=0 \quad \text { when } p>1 .
$$

We determine $b$ by using the values of $\phi$ given above

$$
b=-\frac{Q(0)}{q-n y(0)} \quad \text { if } p=1, \quad \text { or } \quad b=-\frac{Q\left(-\mathrm{p}_{\tau}^{-}\right)}{q-n y\left(-\mathrm{p}_{\tau}^{-}\right)} \quad \text { if } p>1 .
$$


The geometry of $\mathrm{SO}(p) \times \mathrm{SO}(q)$-invariant special Lagrangian cones 247

Similarly (7.36) together with the above values of $\varphi$ implies

$$
\frac{d \widehat{\mathbf{p}}_{\tau}}{d \tau}=\frac{2(n-1)}{q-n y\left(\mathrm{p}_{\tau}\right)} \phi\left(\mathrm{p}_{\tau}\right) \quad \text { if } p=1,
$$

and

$$
\frac{d \widehat{\mathbf{p}}_{\tau}}{d \tau}=\frac{2 p q}{q-n y\left(\mathrm{p}_{\tau}^{+}\right)} \phi\left(\mathrm{p}_{\tau}^{+}\right) \quad \text { if } p>1
$$

Combining these expressions with (7.39) and (7.40) yields (7.25) and (7.26).

Proposition 7.41. For $\tau>0$ the angular period satisfies

$$
\frac{d \widehat{\mathrm{p}}_{\tau}}{d \tau} \sim \frac{4 p}{q} \mathrm{p}_{\tau} \quad \widehat{\mathrm{p}}_{\tau}-\frac{\pi}{2} \sim 2 p \tau \mathrm{p}_{\tau}
$$

Proof. We already proved in $[13$, Prop 7.7$]$ that $\lim _{\tau \rightarrow 0} \widehat{\mathrm{p}}_{\tau}=\frac{\pi}{2}$. The results will now follow easily from Lemma 7.24 by estimating the appropriate values of $Q$ when $\tau$ is sufficiently small.

To achieve this we subdivide the interval $\left(0, \mathrm{p}_{\tau}\right)$ when $p=1$ or $\left(-\mathrm{p}_{\tau}^{-}, \mathrm{p}_{\tau}^{+}\right)$ when $p>1$ as in the proof of 7.3. By Remark 7.29 we obtain bounds on $Q(t)$ independent of $\tau$ except when $y(t)$ is close to $y_{\min }$ and in the case $p>1$ also when $y(t)$ is close to $y_{\max }$. To deal with these regions we notice that away from zeros of $q-n y$ the first order ODE for $Q$ (7.38) can be rewritten as

$$
\left(\frac{Q}{q-n y}\right)^{\cdot}=\frac{1}{(q-n y)^{2}}
$$

Using (3.13) and (7.43) we see that in the vicinity of $y_{\max }$ or $y_{\min }, \dot{Q}$ is close to $(q-n y)^{-1}$, which is close to either $-1 / p$ or $1 / q$ respectively. Using the asymptotics from 7.3 we conclude

$$
Q\left(\mathrm{p}_{\tau}\right) \sim \frac{1}{n-1} \mathrm{p}_{\tau} \quad \text { when } p=1
$$

or

$$
Q\left(-\mathrm{p}_{\tau}^{-}\right) \sim \frac{1}{p} \mathrm{p}_{\tau}^{-} \quad \text { and } \quad Q\left(\mathrm{p}_{\tau}^{+}\right) \sim \frac{1}{q} \mathrm{p}_{\tau}^{+} \quad \text { when } p>1
$$

which together with Lemma 7.24 implies the result for $\frac{d \widehat{\mathbf{p}}_{\tau}}{d \tau}$ claimed. 
Corollary 7.44. Fix admissible integers $p$ and $q$. Define $N \subset\left(0, \tau_{\max }\right)$ by

$$
N:=\left\{\tau \in\left(0, \tau_{\max }\right) \mid \widehat{\mathbf{p}}_{\tau} \in \pi \mathbb{Q}\right\}
$$

Then

(i) $\tau \in N$ if and only if the $(p, q)$-twisted $S L$ curve $\mathbf{w}_{\tau}$ is closed.

(ii) $N$ is a countably infinite dense subset of $\left(0, \tau_{\max }\right)$.

(iii) For $\tau \in N$ the $S O(p) \times S O(q)$-invariant special Legendrian immersion $X_{\tau}: \mathrm{Cyl}^{p, q} \rightarrow \mathbb{S}^{2(p+q)-1}$ factors through a special Legendrian embedding of the closed manifold $\mathrm{Cyl}^{p, q} / \operatorname{Per}\left(X_{\tau}\right)$, where $\operatorname{Per}\left(X_{\tau}\right) \cong \mathbb{Z} \subset$ $\operatorname{Sym}\left(X_{\tau}\right) \subset \operatorname{Diff}\left(\mathrm{Cyl}^{p, q}\right)$ is the following infinite cyclic subgroup:

$\operatorname{Per}\left(X_{\tau}\right)= \begin{cases}\left\langle\left(\mathrm{T}_{k_{0} \mathbf{p}_{\tau}} \circ-\mathrm{Id}_{\mathbb{S}^{n-1}}\right)\right\rangle & \text { if } p=1 \text { and } k_{0} \text { is even and } n \text { is odd; } \\ \left\langle\left(\mathrm{T}_{k_{0} \mathbf{p}_{\tau}} \circ(-1)^{j} \mathrm{Id}_{\mathbb{S} p-1} \circ(-1)^{k} \operatorname{Id}_{\mathbb{S} q-1}\right)\right\rangle & \text { if } p>1 \text { and } k_{0} \text { is even and } n \text { is odd; } \\ \left\langle\mathrm{T}_{2 k_{0} \mathbf{p}_{\tau}}\right\rangle & \text { otherwise; }\end{cases}$

where $j=q / \operatorname{hcf}(p, q), k=p / \operatorname{hcf}(p, q)$ and $k_{0}$ is the order of the rotational period $\widetilde{\mathrm{T}}_{2 \widehat{\mathrm{p}}_{\tau}}$.

Proof. (i-ii): Except for the statement about the density of the subset $N$ this is proved in [13, Thm 7.15]; the density of $N$ follows from the same argument making use of the refined asymptotics for $\widehat{p}_{\tau}$ proved in 7.41 . From 4.49 the condition $\tau \in N$ is equivalent to the condition that the rotational period $\hat{\mathrm{T}}_{2 \widehat{\mathrm{p}}_{\tau}}$ (recall $(3.9)$ ) of $\mathbf{w}_{\tau}$ is of finite order $k_{0}$ (recall Definition 4.48).

(iii): this follows by combining the structure of $\operatorname{Per}\left(X_{\tau}\right)$ given in 5.44 with 4.44 .

\section{Acknowledgments}

N.K. would like to acknowledge the Leverhulme Trust for funding his visit to Imperial College London in Spring 2009, the Department of Mathematics at Imperial for the supportive research environment during this visit and the NSF for support of his research under DMS grant number 1105371. M.H. would like to thank the EPSRC for their continuing support of his research under Leadership Fellowship under EP/G007241/1. 
The geometry of $\mathrm{SO}(p) \times \mathrm{SO}(q)$-invariant special Lagrangian cones 249

\section{References}

[1] V.I. Arnold, Ordinary differential equations, Universitext, SpringerVerlag, Berlin, 2006, Translated from the Russian by Roger Cooke, Second printing of the 1992 edition. MR 2007b:34001.

[2] R. Bryant, Personal communication, 2010.

[3] E. Carberry and I. McIntosh, Minimal Lagrangian 2-tori in $\mathbb{C P}^{2}$ come in real families of every dimension, J. Lond. Math. Soc. (2) 69(2) (2004), 531-544. MR 2040620.

[4] I. Castro, H. Li, and F. Urbano, Hamiltonian-minimal Lagrangian submanifolds in complex space forms, Pacific J. Math. 227(1) (2006), 43-63. MR 2007k:53092.

[5] D. Gorenstein, Finite Groups, Harper \& Row Publishers, New York, 1968. MR 0231903.

[6] M. Haskins, Constructing special Lagrangian cones, PhD thesis, University of Texas at Austin, 2000.

[7] _ The geometric complexity of special Lagrangian $T^{2}$-cones, Invent. Math. 157(1) (2004), 11-70. MR 2005m:53085.

[8] _ Special Lagrangian cones, Amer. J. Math. 126(4) (2004), 845-871. MR 2075484.

[9] M. Haskins and N. Kapouleas, Higher dimensional special Lagrangian cones by gluing $S O(p) \times S O(p)$-invariant cones, In preparation.

[10] _ Special Lagrangian cones by gluing $S O(n-1)$-invariant cones, In preparation.

[11] Special Lagrangian cones with higher genus links, Invent. Math. 167(2) (2007), 223-294. MR 2270454.

[12] _ Gluing constructions of special Lagrangian cones, Handbook of geometric analysis. No. 1, Adv. Lect. Math. (ALM), vol. 7, Int. Press, Somerville, MA, 2008, pp. 77-145. MR 2483363.

[13] Closed twisted products and $\mathrm{SO}(p) \times \mathrm{SO}(q)$-invariant special Lagrangian cones, Comm. Anal. Geom. 20(1) (2012), 95-162. MR 2903102.

[14] I. Martin Isaacs, Algebra, Brooks/Cole Publishing Co., Pacific Grove, CA, 1994. MR 95k:00003. 
[15] D. Joyce, Special Lagrangian $m$-folds in $\mathbb{C}^{m}$ with symmetries, Duke Math. J. 115(1) (2002), 1-51. MR 1932324.

[16] N. Kapouleas, Constant mean curvature surfaces in Euclidean threespace, Bull. Amer. Math. Soc. (N.S.) 17(2) (1987), 318-320. MR 88g:53013.

[17] _ Complete constant mean curvature surfaces in Euclidean threespace, Ann. Math. (2) 131(2) (1990), 239-330. MR 93a:53007a.

[18] _ Slowly rotating drops, Comm. Math. Phys. 129(1) (1990), 139-159. MR 91c:76024.

[19] Compact constant mean curvature surfaces in Euclidean threespace, J. Diff. Geom. 33(3) (1991), 683-715. MR 93a:53007b.

[20] H.-V. Lê, A minimizing deformation of Legendrian submanifolds in the standard sphere, Differential Geom. Appl. 21(3) (2004), 297-316. MR 2005g:53171.

[21] I. McIntosh, Special Lagrangian cones in $\mathbb{C}^{3}$ and primitive harmonic maps, J. London Math. Soc. (2) 67(3) (2003), 769-789. MR 1967705.

[22] R.M. Schoen, The existence of weak solutions with prescribed singular behavior for a conformally invariant scalar equation, Comm. Pure Appl. Math. 41(3) (1988), 317-392. MR 89e:58119.

[23] L. Simon, Lectures on geometric measure theory, Proceedings of the Centre for Mathematical Analysis, Australian National University, vol. 3, Australian National University Centre for Mathematical Analysis, Canberra, 1983. MR 87a:49001.

[24] A. Strominger, S.-T. Yau, and E. Zaslow, Mirror symmetry is T-duality, Nuclear Phys. B 479(1-2) (1996), 243-259. MR 97j:32022.

Department of Mathematics

South Kensington Campus

IMPERIAL COLLEGE LONDON

E-mail address: m.haskins@imperial.ac.uk

Department of Mathematics

BROWN UNIVERSITY

Providence

RI 02912, USA

E-mail address: nicos@math.brown.edu

RECEIVED FEBRUARY 24, 2012 